\title{
Adaptive Control Schemes for Three-phase Grid-Connected Inverters in Photovoltaic Power Generation Systems
}

\author{
A Thesis \\ Presented to \\ the Faculty of the School of Engineering and Applied Science \\ UNIVERSITY OF VIRGINIA \\ In Partial Fulfillment \\ of the Requirements for the Degree \\ Master of Science in Electrical Engineering
}

by

WANSHI HONG

July 2017 


\section{APPROVAL SHEET}

This thesis is submitted in partial fulfillment of the

requirements for the degree of

Master of Science in Electrical Engineering

Wanshi Hong, Author

This thesis has been read and approved by the examining committee:

Professor Gang Tao, Thesis Advisor

Professor Zongli Lin

Professor Harry Powell

Accepted for the School of Engineering and Applied Science:

Dean, School of Engineering and Applied Science

July, 2017 


\section{Acknowledgments}

I would like to express my special gratitude to my advisor Professor Gang Tao. I would never have finished this thesis without his patient guidance and persistent encouragement. His professional knowledge on control theory helps me to overcome many difficulties that I met in completing this thesis. It is a great privilege to have studied under his guidance.

Secondly, I would also like to thank my research group members and my friends who have been continuously encouraging me and sharing ideas with me.

Finally, I must express my very profound gratitude to my parents for providing me with unfailing support and continuous encouragement throughout my years of study and during the process of researching and writing this thesis. This accomplishment would not have possible without them. 


\section{Abstract}

Control of the three-phase photovoltaic (PV) inverters has drawn increasing attention in recent years for the development of solar energy technology. The fast development of signal processing techniques has made advanced control methods possible for the inverter control system. With the rapid growth of the usage of renewable energy, there have been more performance requirements proposed for PV inverter systems. This thesis studies the control problem for the three-phase PV grid-connected inverters. In particular, an adaptive control method is adopted to deal with system uncertainties in such a problem. In this research, the state-space model of the inverter system, which considers the uncertainties of system parameters, is established. Then two adaptive control designs to solve the control problem are developed: a state-feedback output-tracking adaptive control design and an outputfeedback output-tracking adaptive control design. To eliminate the disturbance influence from the grid side, the adaptive controller is further designed to have the ability to compensate such effects to achieve desired adaptive and asymptotic disturbance rejection. Compared with a classical fixed-gain control method, the adaptive control method is capable of efficiently handling system uncertainties and dealing with some unknown disturbances. Analysis and simulation results have shown the effectiveness of the proposed adaptive control schemes. 


\section{Contents}

1 Introduction 1

1.1 Research Motivation . . . . . . . . . . . . . . . . . 1

1.1.1 Background of Photovoltaic Power Generation . . . . . . . 1

1.1.2 Conventional Control Methods and Drawbacks . . . . . . . . 4

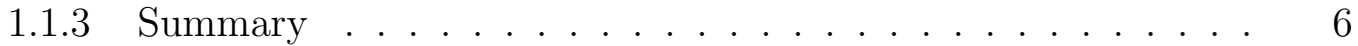

1.2 Literature Review . . . . . . . . . . . . . . . . . . . . . 6

1.3 Thesis Outline . . . . . . . . . . . . . . . . . . . . 8

2 Background $\quad 9$

2.1 Control System Models . . . . . . . . . . . . . . . . . . . . . . 9

2.1 .1 Linear Systems . . . . . . . . . . . . . . . . . . . . . 9

2.1 .2 Nonlinear Systems . . . . . . . . . . . . . . . . . . . 12

2.2 Signal Measures . . . . . . . . . . . . . . . . . . . . . . 14

2.3 System Stability . . . . . . . . . . . . . . . . . . . 15

2.4 Classical Control . . . . . . . . . . . . . . . . . . . . . 17

2.5 Adaptive Control . . . . . . . . . . . . . . . . . 18

3 Three-Phase PV Inverter System Modeling 21

3.1 System Description . . . . . . . . . . . . . . . . . . . . . 21

3.2 State-of-the-Art of Inverter Control … . . . . . . . . . . . 31 
3.3 System Dynamic Equations . . . . . . . . . . . . . . . . . . 33

3.4 Control Problem Formulation . . . . . . . . . . . . . 37

4 State Feedback Adaptive Control Scheme 41

4.1 Problem Formulation . . . . . . . . . . . . . . . . . . . 41

4.2 Basic Design Conditions . . . . . . . . . . . . . . . . . 43

4.3 Adaptive Control for Inverters without Disturbance . . . . . . . . . . 46

4.3.1 Nominal Control Design . . . . . . . . . . . . . 46

4.3.2 Adaptive Control Design . . . . . . . . . . . . . . 49

4.4 Adaptive Control for Grid-Connected PV Inverter . . . . . . . . . . . 52

4.4.1 Nominal Control Design . . . . . . . . . . . . . . . 53

4.4.2 Adaptive Disturbance Rejection Design . . . . . . . . . . . . . 58

4.5 Simulation Results . . . . . . . . . . . . . . . 61

4.5.1 Inverter System Operating without Disturbance . . . . . . . . 62

4.5.2 Inverter System Grid-Connected . . . . . . . . . . . . . . . . 67

4.6 Summary . . . . . . . . . . . . . . . . . 74

5 Output Feedback Adaptive Control Scheme $\quad 75$

5.1 Problem Formulation . . . . . . . . . . . . . . . . . . 75

5.2 Basic Design Conditions . . . . . . . . . . . . . . 77

5.3 Adaptive Control Design for Three-Phase PV Inverter without Distur-

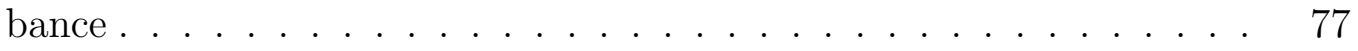

5.3.1 Nominal Control Design . . . . . . . . . . . . . . . . 78

5.3.2 Output Feedback Adaptive Control design . . . . . . . . . . . 80

5.4 Adaptive Control Design for Three-Phase Grid-connected PV Inverter 82

5.4.1 Nominal Control Design . . . . . . . . . . . . . 83

5.4.2 Output Feedback Adaptive Control design . . . . . . . . . . . 85 
5.5 Simulation Results . . . . . . . . . . . . . . . . . . . . . 88

5.5.1 Inverter System Operating without Disturbance . . . . . . . 88

5.5.2 Inverter System Operating in Grid Connected Mode . . . . . . 93

5.6 Summary . . . . . . . . . . . . . . . . . . . 99

6 Conclusions and Future Topics 101

6.1 Summary and Conclusions . . . . . . . . . . . . . . . . . 101

6.2 Future Research Topics . . . . . . . . . . . . . . . . . . 102 


\section{List of Figures}

2.1 Block diagram of an output feedback system with a gain $k$ [15] . . . 17

2.2 Block diagram of a control system with state feedback [15] . . . . . . 18

3.1 Adaptive control system for the PV inverter. . . . . . . . . . . . . 22

3.2 P-V characteristics of the ideal PV panels [9]. . . . . . . . . . . . . . 24

3.3 Flow chart of the MPPT method to determine $P^{*}[9] \ldots \ldots$. . . . . 25

3.4 The generation of SPWM waveforms [16]. . . . . . . . . . . 27

3.5 Analog scheme for SPWM implementation [16]. . . . . . . . . . . 28

3.6 Three-phase SPWM waveforms [16]. . . . . . . . . . . . . . . 28

3.7 Eight switch states $[16] . \ldots \ldots \ldots \ldots$

3.8 Voltage vector space $[16] \ldots \ldots \ldots \ldots$

3.9 Scheme diagram for inverter-grid connecting process. . . . . . . . . . 31

3.10 Structure of a three phase inverter . . . . . . . . . . . . . . . 33

4.1 System response for Case 1. . . . . . . . . . . . . . 65

4.2 System response for Case 2. . . . . . . . . . . . . 66

4.3 System response for Case 3. . . . . . . . . . . . . . . 69

4.4 FFT analysis for the output current $I_{g a}$ for Case $3 . \quad$. . . . . . . . . 70

4.5 System response for Case 4. . . . . . . . . . . . . . . . . . . . 71

4.6 FFT analysis for the output current $I_{g a}$ for Case $3 . \quad$. . . . . . . . . 72 
4.7 System response for Case 5. . . . . . . . . . . . . . . . 73

4.8 FFT analysis for the output current $I_{g a}$ for Case 5. . . . . . . . . . 74

5.1 System response for Case 1. . . . . . . . . . . . . . . . . . . 91

5.2 System response for Case 2. . . . . . . . . . . . . . . . . . . . 92

5.3 System response for Case 3. . . . . . . . . . . . . . . . . 95

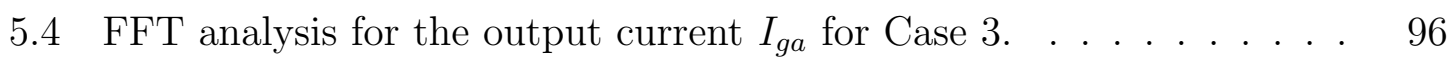

5.5 The output current and tracking error of $I_{g d}, I_{g q}$ for Case $4 \ldots \ldots$. . . 97

5.6 System response for Case 5. . . . . . . . . . . . . . . . . . . 98

5.7 FFT analysis for the output current $I_{g a}$ for Case 5. . . . . . . . . . . 99 


\section{Chapter 1}

\section{Introduction}

This chapter gives a brief introduction about the photovoltaic (PV) inverter. First, the background about renewable energy and some conventional control methods for the inverter system are introduced. Following the PV inverter background, the literature review and the thesis outline are presented.

\subsection{Research Motivation}

This section gives a brief introduction about the Photovoltaic inverter system and some conventional ways for inverter control.

\subsubsection{Background of Photovoltaic Power Generation}

Solar energy is the inexhaustible and eternal energy of the earth. Most of the used energy comes directly or indirectly from the sun. Every year, about $1.8 \times 10^{18} \mathrm{kWh}$ of energy is radiated from the sun to the earth, that is about 10 thousands times of power consumption of the earth. Being one of the most attractive renewable energy, the use of photovoltaic (PV) power has become a trend around the world. 
By the end of 2015, the USA ranked $4^{\text {th }}$ in the world behind China, Germany, and Japan. As of the end of 2016, the U.S. had 40GW of installed photovoltaic capacity, having almost doubled in capacity from the previous year [1]. In the twelve months through January 2017, utility-scale solar power generated 35.5TWh, $0.92 \%$ of total U.S. electricity. There are plans to build other large solar power plants in the U.S., and many states have set their renewable energy targets, where the usage of solar power is included in various proportions. Governor Jerry Brown has signed legislation requiring California's utilities to obtain 50 percent of their electricity from renewable energy sources by the end of 2030 [2]. All these figures show the important strategic position of the solar energy in the field of power generation.

PV Inverter A PV inverter converts the direct current (DC) of a photovoltaic power generation device (solar panel) into a utility frequency alternating current (AC) that can be fed into a grid or used by an off-grid load [24]. Without a doubt, a PV inverter is the core of any PV power generation system (grid-connected or off-grid). Since the grid inverter is, the primary equipment of photovoltaic gridconnected systems, the research focus is the control strategy for grid inverters. The most important standard of the grid inverter is the quality of its output current. The IEEE 1547 standard in the USA allows a limit of 5\% for the current total harmonic distortion (THD) factor with individual limits of $4 \%$ for each odd harmonic from $3^{\text {rd }}$ to $9^{\text {th }}$ and $2 \%$ for $11^{\text {th }}$ to $15^{\text {th }}$ while the European standard IEC61727 suggests a similar one [3].

Grid-Connected PV Systems A grid-connected PV system is connected to a large public grid (owned by a utility company) and feeds power into the grid. Gridconnected systems vary in size from residential $(2-10 \mathrm{~kW})$ to solar power stations (1$10 \mathrm{MW})$. In the case of residential or building mounted grid-connected PV systems, 
the electricity demand of the building is met by the PV system. Only the excess is fed into the grid when there is an excess.

The feeding of PV-generated electricity into the grid requires the transformation of $\mathrm{DC}$ into $\mathrm{AC}$ by a grid-controlled inverter. On the $\mathrm{AC}$ side, the function of the grid-connected inverter is to supply electricity in sinusoidal form, synchronized to the grid frequency, and limit the feed-in voltage to no higher than the grid voltage. On the DC side, because the power output of a module varies as a function of the voltage, power generation can only be optimized by varying the system voltage to find the "maximum power point". Most inverters, therefore, incorporate "maximum power point tracking" [20].

Stand-Alone PV Systems A stand-alone system does not have a connection to the grid, and thus the generated power is stored and buffered with a battery. It is most suitable for locations such as remote villages, mountains, islands and base stations where it is hard to access electricity. A charge controller may be incorporated into the system to avoid battery damage and to optimize the power production of the cells or modules by maximum power point tracking (MPPT). In most applications, an inverter is needed to convert the stored energy in the battery into AC electrical power. Hence, the stand-alone PV system, in a combination of battery and inverter, acts like a self-sufficient system that can provide independent energy supply for long periods of time [20].

Major Problems of Current Grid Connected PV system Nowadays there has been a lot PV systems related research dealing with the following problems.

(i) Randomness of the energy source. For renewable power source, random effect such as temperature, environment, or light intensity can influence the output 
power of the system. Therefore, to control the PV system, the controller for the inverter should have the ability to track an given reference signal which might vary through time.

(ii) The output power quality. The output power quality of the PV system can be shown by the following aspect: Harmonic pollution. For power generation systems, the power electronic devices might cause the increase of the grid current harmonic. A system with lower harmonic contents will be considered to have a better power quality.

(iii) Protection function. The PV system should have the ability to detect fault and have some protection functions such as anti-islanding protection [21]. An islanding effect means that when the grid is powered off but the PV system did not detect this and continue working alone. This will cause danger to customers

and workers of the power company. Other protections such as the isolation of AC and DC units should also be applied to prevent the inverter causing damage to the grid.

\subsubsection{Conventional Control Methods and Drawbacks}

Most common way to control the inverter is proportional plus integral (PI) control. At first, people used PI controllers for the inverter system. However, for the PV inverter with an inductor-capacitor-inductor (LCL) filter, just by using a PI controller may not meet the harmonic standard for the output current. Then researchers come up with a method to combine the PI controller with a proportional resonant controller. This approach can decrease the harmonics for the inverter system.

\section{PI Based Control}


PI control. The proportional plus integral (PI) controller is the most widely used method for PV inverter systems due to its easy implementation. However, the classical PI controller can only achieve the zero steady-state error control for the fundamental frequency of the grid, that is, for tracking constant current values. Only using the PI controller, the current harmonic can not be reduced. These kinds of harmonics, if connected to the grid, will cause some serious problem to the grid.

$P I+P R$ control. Since the classical PI controller can only achieve the zero-steady state error control for the fundamental frequency of the grid, there are some recent approaches by combining the proportional resonant (PR) controller with the traditional PI controller to reduce the certain order of harmonic. But the controller structure would be much more complicated for the three phase grid-connected inverter [25].

Moreover, a classical controller cannot make the output current track a time varying reference signal. This might cause some errors in the PV inverter system due to the randomness of the renewable energy source.

Issues with System Uncertainties Another main drawback for using the classical PI controllers is that this type of controllers cannot effectively deal with system uncertainties, and this might influence the performance of the system. There are mainly two uncertain situations: (a) The uncertainties of system parameters, such as resistance or inductance. Due to the sudden connection to the grid and the change of the output current of the photovoltaic generation device, the change of the system current might change the value of resistance, inductance, etc. Also, the exact value of the equivalent resistance in the system is usually hard to be measured. For example, the equivalent resistance of the connecting wire and other measuring devices in the system. (b) The uncertainty of the output voltage of the photovoltaic generation device, such as solar panels. Ideally, this voltage is expected to be a constant value 
which is a parameter in the inverter system dynamic model (see Section 3.3) The uncertainty of this parameter is due to the uncertainty from the intensity of the sunlight.

\subsubsection{Summary}

From the discussion above, it can be seen that developing a reliable controller with the ability to deal with system uncertainties and achieve signal tracking is necessary for the grid-connected photovoltaic system. This motivated our research on using multivariable model reference adaptive control (MRAC) methods which are capable of dealing with uncertain system parameters and rejecting unknown disturbances. For this type of applications, the results of this research are reported in this thesis.

In this thesis, we will study and develop some new solutions to the following problems:

Problem I: The state feedback output tracking model reference adaptive control (MRAC) scheme for the inverter system working in the grid-connected mode;

Problem II: The output feedback output tracking MRAC scheme for the inverter system working in the grid-connected mode;

Problem III: The adaptive control schemes for the inverter system connected to the polluted grid, that is, the power grid with a high content of high order harmonic components and some unknown voltage change of the grid.

\subsection{Literature Review}

The control strategy for the three-phase grid connected photovoltaic system has been a research hotspot for years. There are some amazing improvements of the 
control strategy for this system to make a better grid-connected performance, and there has been some research developing some useful models for this control topic.

PV Inverter Systems and Control In [7], the authors eliminated the high order harmonics of the input by applying a proportional-resonant $(\mathrm{PR})$ controller operating with the classical PI controller. The function of a PR controller is to eliminate the harmonics in a particular frequency, in which frequency the harmonic component cannot be reduced by the PI controller. This has been a suitable method for the gridconnected photovoltaic system. In this research, they give a specific modeling method to the inverter system. For the three-phase inverter system, this control structure is rather complicated due to the complexity of the three-phase system. This method achieved the elimination of the current harmonics and the basic control objectives of an inverter system.

In [17], they discussed the control structure for the PV system by using an LCL filter in the system. Since an LCL filter can improve the efficiency of filtering and also reduce the volume of the filter, some useful control models are given for PV system with an LCL filter. In this paper, the significance of the LCL filter is mentioned. They used a classical control method for the inverter control problem, and they did not consider the harmonic problem for this system. However, with this improvement in the grid-connected PV system, the controller has become harder to design since the order of the filter has increased.

In [19], a nonlinear control for the three-phase inverter using the derivative-free nonlinear Kalman filter is introduced, this paper develops a nonlinear approach to the three-phase inverter system with a Kalman filter. To make the control problem simpler, in this research they directly control the power to track the desired reference value which is obtained from the maximum power point tracking (MPPT) module. 
Adaptive Control In [5], [6], [18], some adaptive approaches to this control problem are given. In [18], they used an adaptive control scheme to help predict the system parameter when the system is working under polluted environment. However, the authors still use the PI+PR controller for the control problem of the main circuit. In [5], an adaptive droop control is also used to help predict the system parameters. A similar adaptive control method with the one in this research is developed in [6] but only the off-grid case is considered, and the case where the system has an LCL filter is not considered.

\subsection{Thesis Outline}

The remainder of the thesis is organized as follows. Chapter 2 introduces a basic information for understanding this thesis including the background of the control systems and the background for adaptive control. Chapter 3 provides specific introduction to each module of the inverter system and how the inverter system operates, then presents the modeling of the inverter system. In Chapter 4 , the state feedback output tracking adaptive control schemes for the grid-connected inverter system as well as the simulation results of this control scheme are presented. In Chapter 5, the output feedback output tracking adaptive control schemes for the grid connected inverter is proposed, and the simulation result of this control scheme follows. Finally, in Chapter 6, the conclusions and future work for this thesis are discussed. 


\section{Chapter 2}

\section{Background}

Before starting to discuss the adaptive control of three phase inverter system, some basic background about control systems needs to be presented. In this chapter, topics about control systems including control system modeling, system stability, classical control and adaptive control are presented.

\subsection{Control System Models}

Control system models are derived from the differential equations that describe the input-output behavior of a system.

\subsubsection{Linear Systems}

There are two approaches to developing control system models. The first being what has become known as the classical approach and the second being the so-called modern approach [8]. 
Classical Approach to Modeling A general $n$ th-order linear time invariant differential equation has the form

$$
\frac{d^{n} y(t)}{d t^{n}}+a_{n-1} \frac{d^{n-1} y(t)}{d t^{n-1}}+\cdots+a_{0} y(t)=b_{m} \frac{d^{m} u(t)}{d t^{m}}+b_{m-1} \frac{d^{m-1} u(t)}{d t^{m-1}}+b_{0} u(t),
$$

where $a_{i}$ and $b_{i}$ are the system parameters. The classical approach to obtaining a model of a system is first to find the differential equations describing the system. Once the equations have been obtained, the Laplace transform is used to turn the nth-order differential equations dependent on the time $t$ into an $\mathrm{n}^{\text {th }}$-order algebraic equation dependent on the complex variable $s$. At this point, it is easy to manipulate the equation to obtain an input-output description of the system referred to as the system transfer function [8].

Modern Approach to Modeling The current approach to modeling a system is more commonly known as the state-space approach. The state-space model of a system overcomes some of the limitations of the transfer function. The most important distinctions between the two are that nonlinear, time-varying, and multiple-inputmultiple-output (MIMO) systems can be represented with a state-space model.

In general, four steps need to be taken to derive a state-space model.

Step 1 Choose the state variables. These must be picked so that the minimum number of state variables chosen describe the state of the system completely. More importantly, the state variables must be linearly independent of one another.

Step 2 Find $n$ simultaneous first order differential equations regarding the state variables. These equations are known as the state equations.

Step 3 This step depends on whether knowledge of the input for $t \geq t_{0}$ and the initial condition of each state variable at an initial time $t_{0}$ is available. If this 
information is available, then the state equations can be solved for the state variables.

Step 4 Algebraically combine the state variables with the systems input to find all other system variables for $t \geq t_{0}$. These equations are known as the output equations.

The state equations and output equations found in Steps 2 and 4 are known collectively as the state-space model of a system [8].

With the process of how to select the state variables and derive the state and output equations given, it is important to define how a state-space model is commonly represented. The derivative of the state variables on time is taken and placed in a vector, $\dot{x}(t) \in R^{n}$. The state variables are taken and placed into a vector, $x(t) \in R^{n}$, called the state vector. The coefficients relative to a state variable for each differential equation are taken and placed in a matrix, $A \in R^{n \times n}$, called the system matrix. The coefficients relative to the input for each differential equation are placed in a matrix, $B \in R^{n \times M}$, called the input matrix. The input is denoted as $u(t) \in R^{M}$. The output is denoted as $y(t) \in R^{M}$. The coefficients relative to any of the unsolved for variables in the output equation are taken and placed in a matrix, $C \in R^{r \times n}$, called the output matrix. For some systems there is a matrix, $D \in R^{r \times M}$, called the feed-forward matrix that contains any feed-forward terms in the system. For this research, the feed-forward matrix will consist entirely of zeros since there are no feedforward terms multiplied by the input. Altogether, the previously described vectors and matrices form the state space representation defined as

$$
\dot{x}(t)=A x(t)+B u(t), y(t)=C x(t)+D u(t), t \geq t_{0} .
$$


In this case, the state solution can be explicitly expressed as

$$
x(t)=e^{A\left(t-t_{0}\right)} x_{0}+\int_{t_{0}}^{t} e^{A(t-\tau)} B u(\tau) d \tau,
$$

where $e^{A t}=\mathcal{L}^{-1}\left[(s I-A)^{-1}\right]$.

\subsubsection{Nonlinear Systems}

An $n$th order dynamic system can be written as one $n$-dimensional first-order vector differential equation

$$
\dot{x}=f(t, x, u), y=h(t, x, u), t \geq t_{0}
$$

where $x \in R^{n}$ is the state vector, and $u \in R^{M}$ is the input vector. The first equation is the state equation while the second equation associated with an $M$-dimensional output vector $y \in R^{M}$. The solution for (2.4) is denoted as $x(t)=x\left(t ; t_{0}, x_{0}\right)$, where $x\left(t_{0}\right)=x_{0}$ is the initial state vector. To analysis the non-linear system, one method is to linearize the nonlinear system at an operating point $\left(x_{0}, u_{0}\right)$ by Taylor expansion, which is

$$
\begin{aligned}
& \delta \dot{x}=A \delta x+B \delta u \\
& \delta y=C \delta x+D \delta u
\end{aligned}
$$


for $f\left(t, x_{0}, u_{0}\right)=0$, with

$$
\begin{gathered}
A=\left.\frac{\partial f}{\partial x}\right|_{\left(x_{0}, u_{0}\right)}=\left.\left[\begin{array}{ccc}
\frac{\partial f_{1}}{\partial x_{1}} & \ldots & \frac{\partial f_{1}}{\partial x_{n}} \\
\vdots & & \vdots \\
\frac{\partial f_{n}}{\partial x_{1}} & \ldots & \frac{\partial f_{n}}{\partial x_{n}}
\end{array}\right]\right|_{\left(x_{0}, u_{0}\right)}, B=\left.\frac{\partial f}{\partial u}\right|_{\left(x_{0}, u_{0}\right)}=\left.\left[\begin{array}{ccc}
\frac{\partial f_{1}}{\partial u_{1}} & \ldots & \frac{\partial f_{1}}{\partial u_{M}} \\
\vdots & & \vdots \\
\frac{\partial f_{n}}{\partial u_{1}} & \ldots & \frac{\partial f_{n}}{\partial u_{M}}
\end{array}\right]\right|_{\left(x_{0}, u_{0}\right)}, \\
C=\left.\frac{\partial h}{\partial x}\right|_{\left(x_{0}, u_{0}\right)}=\left.\left[\begin{array}{ccc}
\frac{\partial h_{1}}{\partial x_{1}} & \ldots & \frac{\partial h_{1}}{\partial x_{n}} \\
\vdots & & \vdots \\
\frac{\partial h_{q}}{\partial x_{n}} & \ldots & \frac{\partial h_{n}}{\partial x_{n}}
\end{array}\right]\right|_{\left(x_{0}, u_{0}\right)}, D=\left.\frac{\partial h}{\partial u}\right|_{\left(x_{0}, u_{0}\right)}=\left.\left[\begin{array}{ccc}
\frac{\partial h_{1}}{\partial u_{1}} & \ldots & \frac{\partial h_{1}}{\partial u_{M}} \\
\vdots & & \vdots \\
\frac{\partial h_{q}}{\partial u_{1}} & \ldots & \frac{\partial h_{q}}{\partial u_{M}}
\end{array}\right]\right|_{\left(x_{0}, u_{0}\right)} .
\end{gathered}
$$

As a result the linearized system is

$$
\begin{aligned}
& \dot{x}=A x+B u \\
& y=C x+D u .
\end{aligned}
$$

This process is called linearization. Such a linearization method is concerned with the local stability of a nonlinear system. It is a formalization of the intuition that a nonlinear system method should behave similarly to its linearized approximation for a small range signals. Because all physical systems are inherently nonlinear, linearization method serves as the fundamental justification of using linear control techniques in practice. More details about the linearization method can be found in literature [12]. 


\section{$2.2 \quad$ Signal Measures}

To measure vector signals, signal norms are defined. Consider a vector signal $x(t)=\left[x_{1}(t), \ldots, x_{n}(t)\right]^{T} \in R^{n}$, which is a vector function as $t$ changes. Vector norms are used to measure vectors at a fixed $t$ while the signal norms can measure the functions for all $t$. The norm concept is defined below:

Definition 2.1. A real-value function $\|\cdot\|$ on a linear space $\mathcal{D}$ is a norm if

(i) $\|x\| \geq 0$ for all $x \in \mathcal{D}$ and $\|x\|=0$ only if $x=0$;

(ii) $\|\alpha x\|=|\alpha|\|x\|$ for all $x \in \mathcal{D}$ and any scalar $\alpha$; and

(iii) $\|x+y\| \leq\|x\|+\|y\|$ for all $x, y \in \mathcal{D}$.

For a vector signal, the signal norm $L^{1}, L^{2}, L^{\infty}$ norms are defined as

$$
\begin{aligned}
\|x(\cdot)\|_{1} & =\int_{0}^{\infty}\|x(t)\|_{1} d t \\
\|x(\cdot)\|_{2} & =\sqrt{\int_{0}^{\infty}\|x(t)\|_{2}^{2} d t} \\
\|x(\cdot)\|_{\infty} & =\sup _{t \geq 0}\|x(t)\|_{\infty},
\end{aligned}
$$

where the vector norms are

$$
\begin{aligned}
\|x(t)\|_{1} & =\left|x_{1}(t)\right|+\cdots+\left|x_{n}(t)\right| \\
\|x(t)\|_{2} & =\sqrt{x_{1}^{2}(t)+\cdots+x_{n}^{2}(t)} \\
\|x(t)\|_{\infty} & =\max _{1 \leq i \leq n}\left|x_{1}(t)\right| .
\end{aligned}
$$




\section{$2.3 \quad$ System Stability}

The concept of stability is crucial to control system design. An unstable control system is useless and dangerous. The methods are available to examine the poles depending on the representation of the system model. If the classical approach is taken, then the poles of the transfer function can be examined. If the modern approach is used then the eigenvalues, which are the system poles, of the system matrix A can be analyzed. Either approach can quickly give information on whether or not the system is asymptotically stable, stable, or unstable.

For adaptive control systems, stability must be defined another way since knowledge of the system parameters are unavailable and possibly changing. The work of Alexander Mikhailovich Lyapunov, who presented definitions and theorems for studying the stability of solutions to a broad class of differential equations, has been used extensively to address this problem [10]. The work of Lyapunov relies on defining an energy function, formally known as a Lyapunov function candidate, that can be used to determine the stability of a system without having to solve for the solutions to the system explicitly. This is the so-called Lyapunov direct method for stability analysis. Originally, this Lyapunov function was purely the total mechanical or electrical energy and therefore by nature positive definite. The Lyapunov indirect method which determines system stability based on its linearized model can be found in many textbooks about a nonlinear system like [11], [12].

Stability Definitions Since all systems to be discussed in this thesis are LTI systems, we will introduce the definition of stability for LTI systems only [13].

Definition 2.2. The response of $\dot{x}(t)=A x(t)$ is stable in the sense of Lyapunov if every finite initial state $x_{0}$ excites a bounded response. It is asymptotically stable if 
every finite state excites a bounded response which, also, approaches 0 as $t \rightarrow \infty$.

Usually, we do not use the definition to check the stability of an LTI system. Theorem 2.1 can help us to check the stability of an LTI system quickly.

Theorem 2.1. The equation $\dot{x}(t)=A x(t)$ is stable if and only if all eigenvalues of A have zero or negative real parts and those with zero real parts are simple roots of the minimal polynomial of $A$. The equation $\dot{x}(t)=A x(t)$ is asymptotically stable if and only if all eigenvalues of $A$ have negative real parts.

Also in the Lyapunov sense, we can check the stability of matrix $A$ by the Lyapunov theorem.

Theorem 2.2. The equation $\dot{x}(t)=A x(t), A \in R^{n \times n}, x \in R^{n}$ is asymptotically stable if and only if for every positive definite $Q=Q^{T} \in R^{n \times n}$, the Lyapunov equation $A^{T} P+P A=-Q$ has a unique and positive definite solution $P=P^{T} \in R^{n \times n}$.

Theorem 2.1 and Theorem 2.2 are theorems which we usually use to check the stability of the close-looped system by classical control. But for adaptive control, Theorem 2.1 and Theorem 2.2 would not work because in adaptive control there exist uncertainties on the dynamics model; we have no access to get a set of accurate system parameters. So we introduce a new method called Lyapunov direct method to help us check the system stability when applying adaptive control.

Theorem 2.3 (Lyapunov direct method). If in some ball $B(h)=\left\{x \in R^{n}: \| x-\right.$ $\left.x_{0} \| \leq h\right\}$ there exists a positive definite function $V(x, t)$ with $\dot{V} \leq 0$, then the equilibrium state $x_{e}=0$ of the system $\dot{x}=f(x, t)$ is stable. If, in addition, $V(x, t)$ is decrescent, then it is uniformly stable.

Now the Barbalart lemma is introduced, which makes the adaptive control system signal convergence analysis easier. 
Lemma 2.1 (Barbalat Lemma). If a scalar function $f(t)$ is uniformly continuous such that $\lim _{t \rightarrow \infty} \int_{0}^{t} f(\tau) d \tau$ exists and is finite, then $\lim _{t \rightarrow \infty} f(t)=0$.

We also have the following lemma:

Lemma 2.2. If a scalar function $f(t)$ satisfy $\dot{f}(t) \in L^{\infty}$, and $f(t) \in L^{2}$, then $\lim _{t \rightarrow \infty} f(t)=0$.

This lemma is readily used for adaptive control system signal convergence analyze.

\subsection{Classical Control}

The first thing we need to know about the classical control is the feedback is pervasive [14]. Feedback is a very crucial method to stabilize the unstable system stable. Usually, the output $y(t)$ is fed back and compared with the input $u(t)$. The block diagram of the most classical feedback control system is shown in Figure 2.1. System transfer function can be computed by block diagrams like Figure 2.1. Remind that in Figure 2.1, $k$ is a constant through the whole control process. Whether the control parameter $k$ can be updated or not is one of a big differences between adaptive control and classical control.

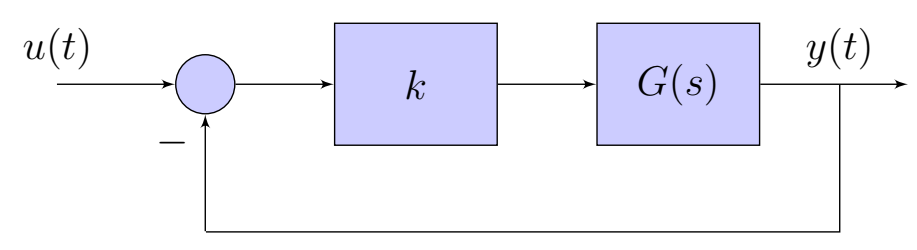

Figure 2.1: Block diagram of an output feedback system with a gain $k$ [15]

With the state space representation it is convenient to feedback the state variables as the components of the state vector $x(t)$ to form the control signal $u(t)$, instead of the feedback of an output signal $y(t)$. With this configuration, each state variable is 
multiplied by a comoinent of a gain vector $K$ to give the desired closed loop poles. A typical control system represented with the state space representation utilizing state feedback is displayed in Figure 2.2 where double error lines represent vector signals.

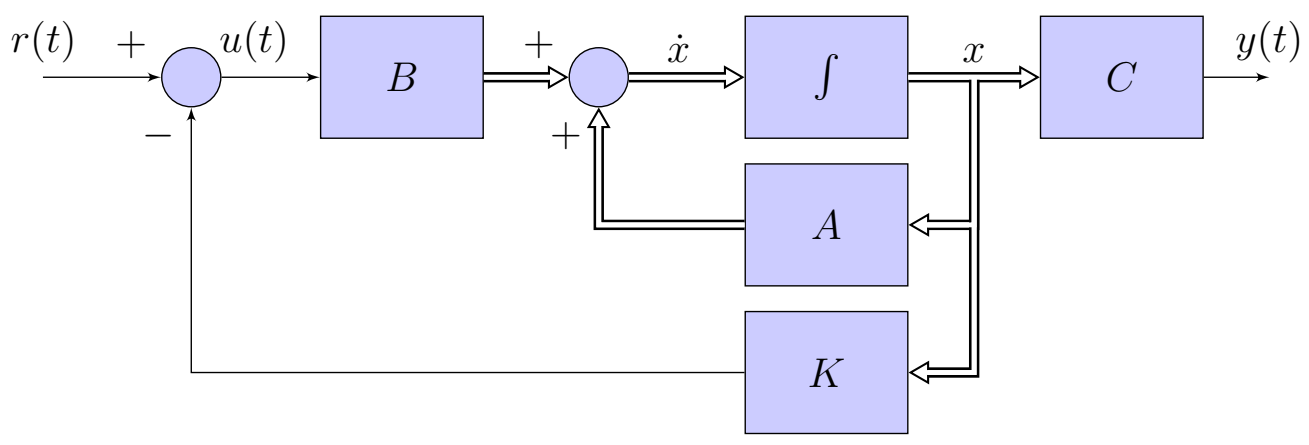

Figure 2.2: Block diagram of a control system with state feedback [15].

\subsection{Adaptive Control}

Adaptive control provides adaptation mechanisms that adjust a controller for a system with parametric, structural, and environmental uncertainties to achieve desired system performance. Payload variation or component aging causes parametric uncertainties. Component failure leads to structural uncertainties and the external noises are typical environmental uncertainties. Such uncertainties often appear in automobile engines, electronic devices, and other industrial processes. Adaptive control has experienced many successes in new challenging problems and their encouraging solutions.

Unlike other controllers using PID, pole placement, optimal, robust or nonlinear control methods, whose designs are based on certain knowledge of the system parameters, adaptive controllers do not need such knowledge of the system parameters; they are adapted to parameter uncertainties by using performance error information on-line [11]. There exist mainly two types of adaptive controllers: state feedback controllers and output feedback controllers. 
State Feedback Design for Output Tracking Consider the linear time-invariant system described in a state-space form as

$$
\dot{x}(t)=A x(t)+B u(t), y(t)=C x(t), t \geq 0,
$$

where $A \in R^{n \times n}, B \in R^{n \times M}$ are constant parameter matrixs, $x(t) \in R^{n}$ is the state vector, $u(t) \in R^{M}$ is the control input, and $y(t) \in R^{q}$ is the output. With the state variables in $x(t)$ available for feedback, the control objective is to design a state feedback control signal $u(t)$ such that all signals in the closed-loop system are bounded and asymptotic output tracking is achieved, without knowledge of the system parameters. A typical state feedback controller structure is

$$
u(t)=K_{1}^{T}(t) x(t)+K_{2}(t) r(t)
$$

where $K_{1}(t) \in R^{n \times M}$ and $K_{2}(t) \in R^{M \times M}$ are the estimates of some ideal controller parameters $K_{1}^{*}$ and $K_{2}^{*}$, which can be obtained with the system parameters known. The task of adaptive control is to generate the parameter estimates $K_{1}(t)$ and $K_{2}(t)$ without the knowledge of $K_{1}^{*}$ and $K_{2}^{*}$ to achieve the control objective.

Output Feedback Design for Output Tracking For the system (2.17), the typycal output feedback adaptive controller structure is

$$
u(t)=\Theta_{1}^{T}(t) \omega_{1}(t)+\Theta_{2}^{T}(t) \omega_{2}(t)+\Theta_{20}(t) y(t)+\Theta_{3}(t) r(t)
$$

where $\omega_{1}(t)=F(s)[u](t), \omega_{2}(t)=F(s)[y](t), F(s)=\frac{A(s)}{\Lambda(s)}, A(s)=\left[I_{m}, s I_{m}, \ldots, s^{\bar{\mu}_{0}-2 I_{M}}\right]$ $\left(\bar{\mu}_{0}\right.$ is the upper bound of the observability index of $\left.G(s)=C(s I-A)^{-1} B\right), \Theta_{1}(t), \Theta_{2}(t)$, $\Theta_{20}(t), \Theta_{3}(t)$ are the estimates of the ideal controller parameters $\Theta_{1}^{*}, \Theta_{2}^{*}, \Theta_{20}^{*}, \Theta_{3}^{*}$, 
which can be calculated if the system parameters in $G(s)$ are known. The adaptation of the controller parameters is based on the performance error $y(t)-y_{m}(t)$ such that the closed loop system adjusts itself toward an operation condition that which the desired system performance is achieved asymptotically: $\lim _{t \rightarrow \infty} y(t)-y_{m}(t)=0$, where $y_{m}(t)$ is the reference signal for the output $y(t)$.

There are two approaches to adaptive control design. The first approach is known as Direct Adaptive Control and is characterized by the ability to update the controller parameters online through the use of an adaptive update law without initially determining the characteristics of the plant and the possible disturbances. The second approach is known as Indirect Adaptive Control and is characterized by first estimating the parameters of the plant being controlled as well as the possible disturbances, then updating the controller based on a design equation. Direct adaptive control algorithms will be employed in this thesis. More information about adaptive control methods can be found in [11], [31], [32]. 


\section{Chapter 3}

\section{Three-Phase PV Inverter System Modeling}

This chapter first gives an introduction to each module of the inverter system and an over view of the state-of-the-art for inverter control techniques. Then the modeling of the inverter system is derived. By discussing the requirement of the inverter system, the control problem is proposed.

\subsection{System Description}

The function of a three-phase inverter is to manipulate the input DC voltage/current with switch signals to make it the desired three-phase AC current. In this section, we will introduce how a grid connected three-phase photovoltaic (PV) inverter operate. Figure 3.1 shows the basic structure of a typical grid connected three phase PV in-

verter, where the subscripts $a, b, c$ in the signals $d_{a, b, c}, i_{i, a, b, c}, U_{c, a, b, c}$ etc. denote three components of each signal. 


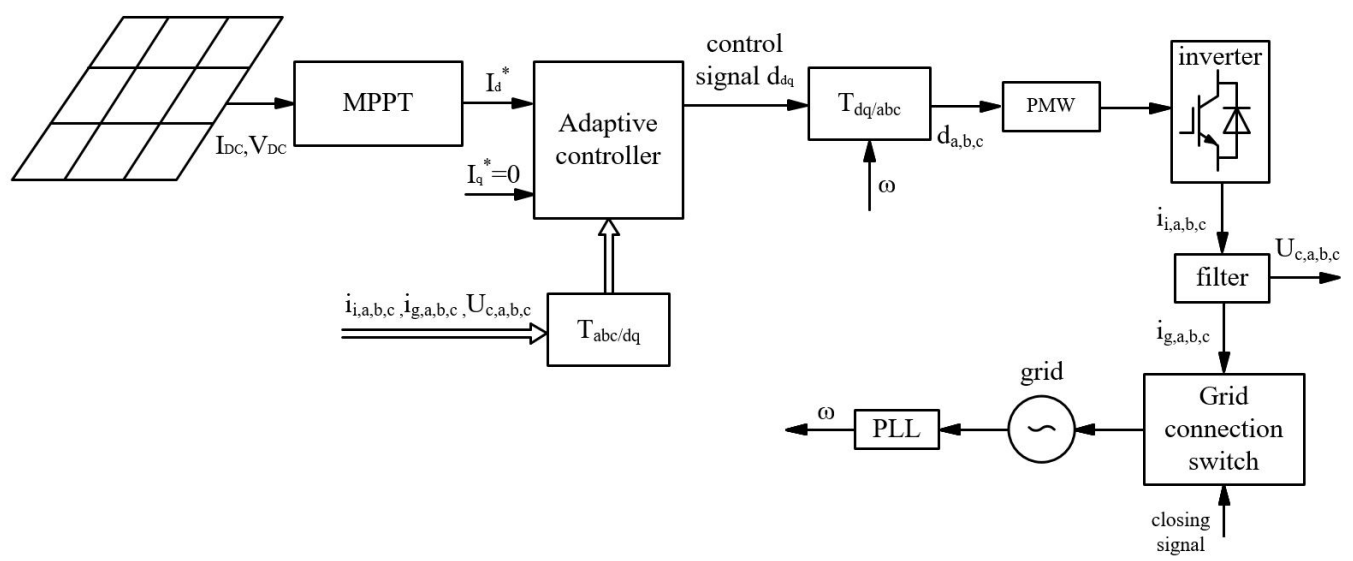

Figure 3.1: Adaptive control system for the PV inverter.

The goal for our adaptive scheme is to design a current controller to generate control signal to control the output inverter current and make the inverter system operating at the maximum power point $P^{*}$. To achieve this goal, our adaptive controller should correspond with each module in the inverter system. We can control the reference signal for (current) $I_{d}$ the inverter output to track is generated from the photovoltaic power generation equipment, such as solar panels. To get the maximum efficiency from the power generation device, the maximum power point tracking (MPPT) unit is used to get the maximum power point $P^{*}$ and thus a maximum $I_{d}^{*}$, which can make the solar panel work at the maximum power point. The inverter output current $i_{i, a, b, c}$ is generated by the inverter using the pulse width modulation (PWM) control. The control signal $u_{a b c}$ represents the duty cycles of the PWM waveform, which can generate switching signals to the insulated gate bipolar transistors (IGBTs) inside te inverter. In this process, the DC side current is changed to the desired AC output current. The output current of the inverter $i_{i, a, b, c}$ goes through an LCL filter to eliminate the high order harmonics. Before connecting to the grid, the output three-phase voltage of the inverter system needs to satisfy that the magnitude, frequency, and phase must be the same as the grid voltage. To this end, a module 
to generate the closing signal is needed. Under the three-phase equilibrium condition and by using the direct-quadrature-zero (DQZ) transformation $T_{a b c / d q}$ (defined in the next section), we can get the circuit differential equations under a rotating coordinate system. To make the output current have same frequency and phase with the grid, a phase lock module (PLL) is needed. This module collects signals from the grid side and then updates their phase and frequency to the controller. This is a necessary process of the DQZ transformation which relies on an accurate frequency update. The function of each module is further introduced in this section.

Maximum Power Point Tracking (MPPT) Despite the control problem of the inverter, the MPPT module is also a crucial part of the inverter system. Figure 3.2 shows the simulated P-V characteristics of the ideal PV panels. The series of curves show the output characteristics under different insolation conditions, which represents the different amount of energy the sun radiates to the earth [9]. By observation we can see that for each insolation conditions, there is a maximum power point within the variation of the voltage. The goal of MPPT is to find the maximum power point $P^{*}$ which is used in the later part of the system to calculate the reference current for the adaptive controller. 


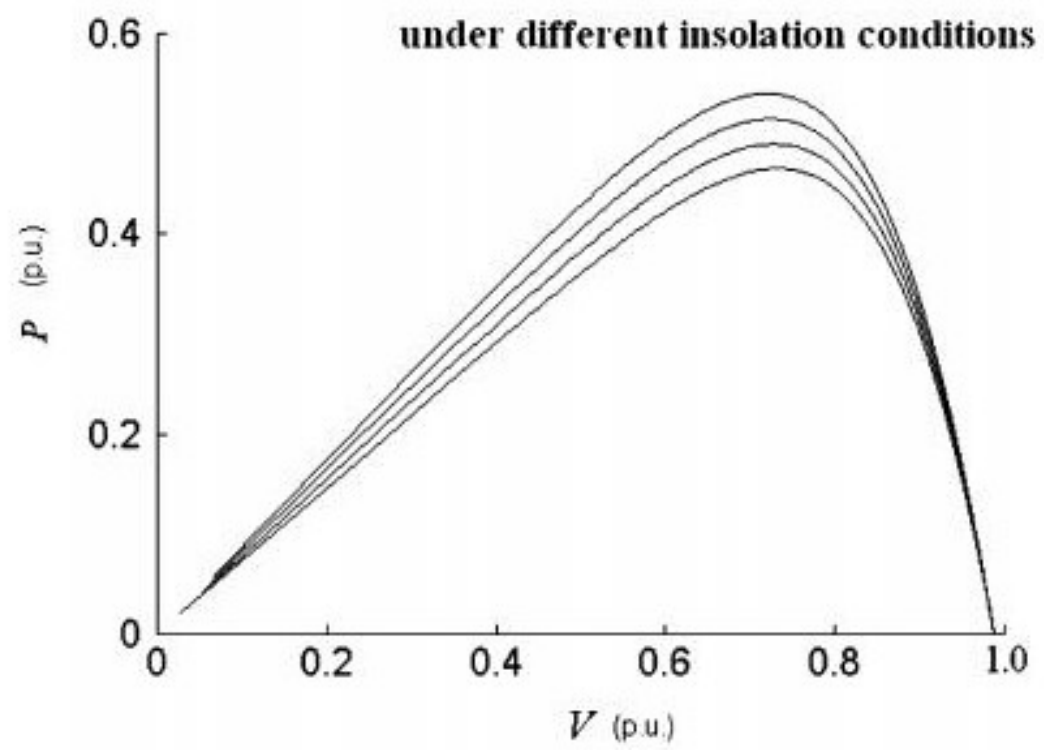

Figure 3.2: P-V characteristics of the ideal PV panels [9].

Define the output current, output voltage and output power of the PV panel as $V_{P V}, I_{P V}, P_{P V}$. Figure 3.3 shows the flow chart to determine $P^{*} . \Delta P$ is the step change of the output power $P, P_{1}$ is the minimum step length to find $P^{*}, K$ is a constant with value usually between 2 and 3 , and $P_{0}$ is the PV output power in the previous control period. This modified MPPT method is so called a constant step MPPT method. 


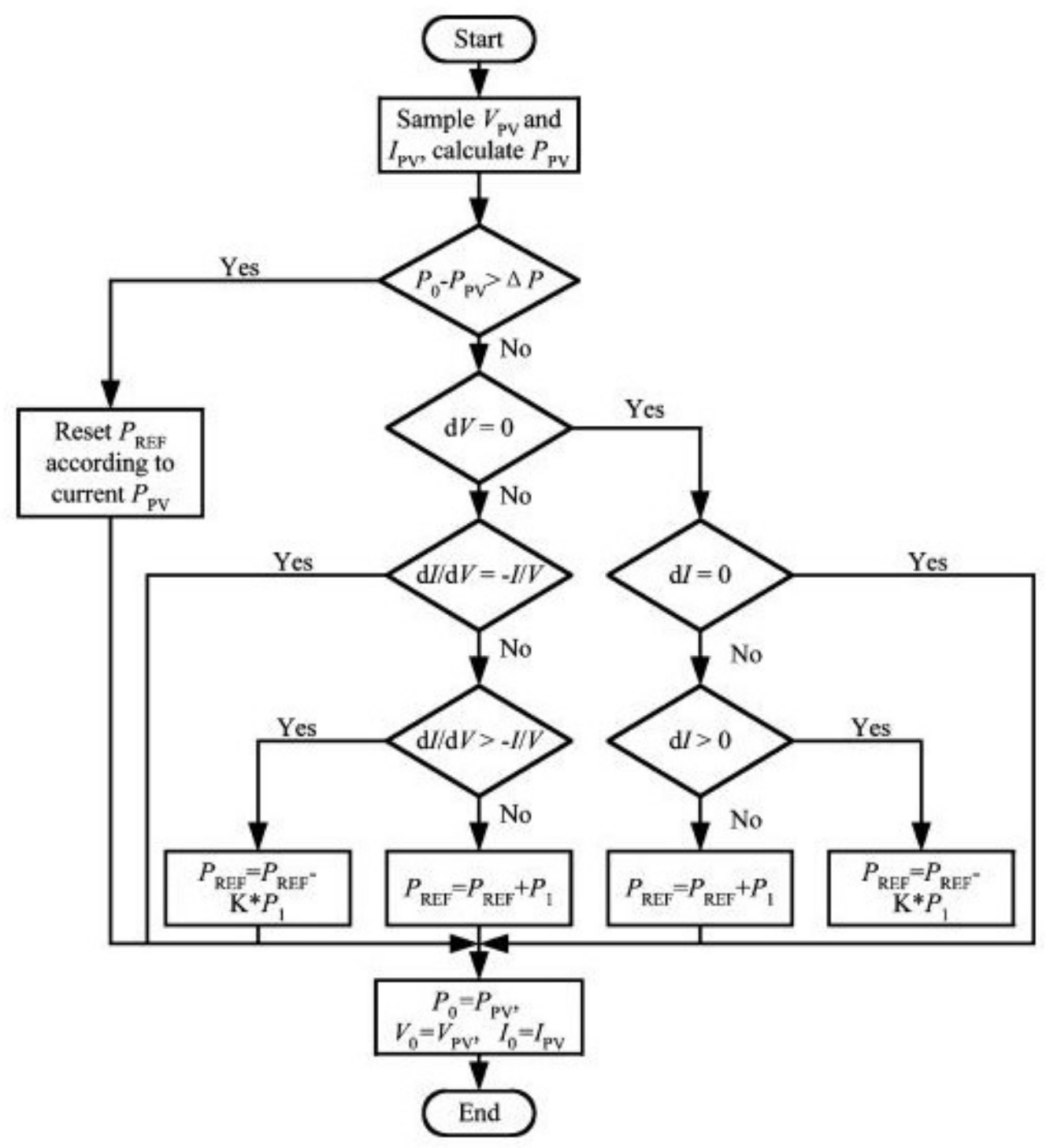

Figure 3.3: Flow chart of the MPPT method to determine $P^{*}[9]$.

LCL filter The conventional three-phase grid-connected inverter uses an LC filter or just an L filter to eliminate the high order harmonics. However with the power level of the inverter advanced to a new level, to eliminate the power loss of the inverter, the switching frequency of the power electronic devices will become lower, this will lead to the increase of high order harmonics of the grid side. As a result, to meet the total harmonic distortion (THD) standard, the inductance will become very high if 
one only use the L filter. This will lead to a series of problems which will cause not only higher cost and larger size for the system, but also the increase of the inductance affecting the system dynamically. The replacement of the L filter by an LCL filter has been one of the most modern solutions for the above problems recently. An LCL filter shows better ability to suppress the high order harmonics and can achieve a better result with a lower total inductance. However, the LCL filter is a third order system with multi-variables, and this will lead to a higher request to the control of this system.

Principle of PWM control [16] The main principle of the PWM (Pulse Width Modulation) technique is: use a series of pulses with the same amplitude but different width which is generated from the semiconductor switching devices (power electronics devices) to replace the sinusoidal wave (SPWM) or other required waveforms. The control signal $d_{a, b, c}(t)$ is the duty cycle of the output waveform. With a certain rule to modulate this duty cycle, both the output voltage and the output frequency of the inverter can be regulated, Figure 3.4 shows the generation of SPWM waveforms. 


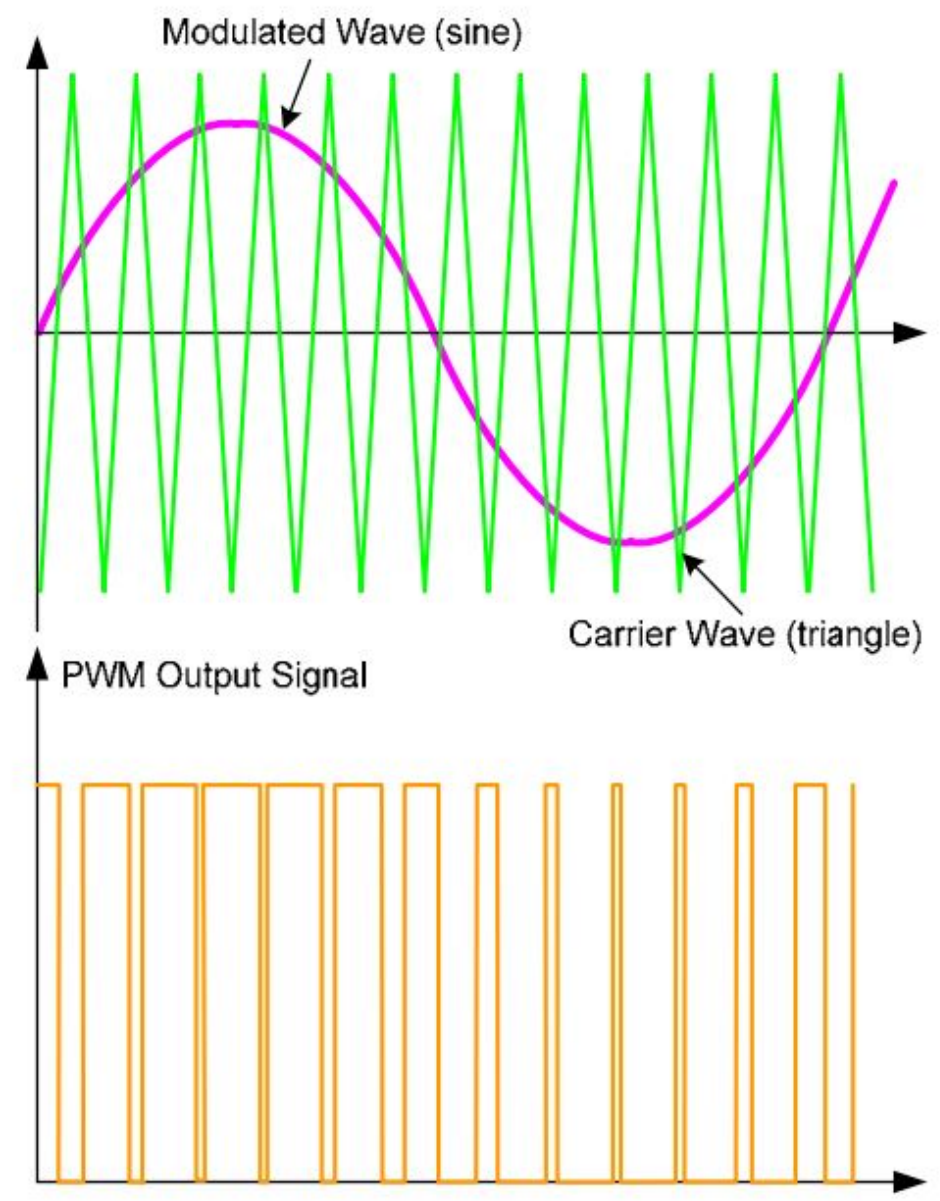

Figure 3.4: The generation of SPWM waveforms [16].

With the basic principle of the SPWM generation above, the analog circuit to implement the principle above is easy to achieve, Figure.3.5 is the analog circuit to generate a desired SPWM waveform. 


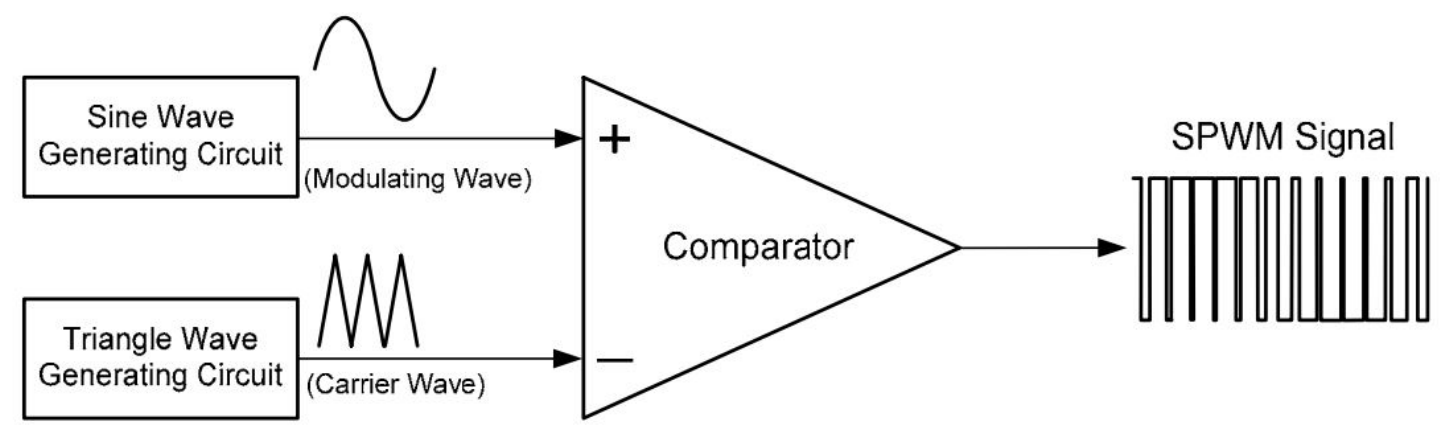

Figure 3.5: Analog scheme for SPWM implementation [16].

For three-phase inverter systems, the SPWM signals to trigger the six power switches are generated by comparison of the three phase since waves with the same triangular wave shown in Figure 3.6.

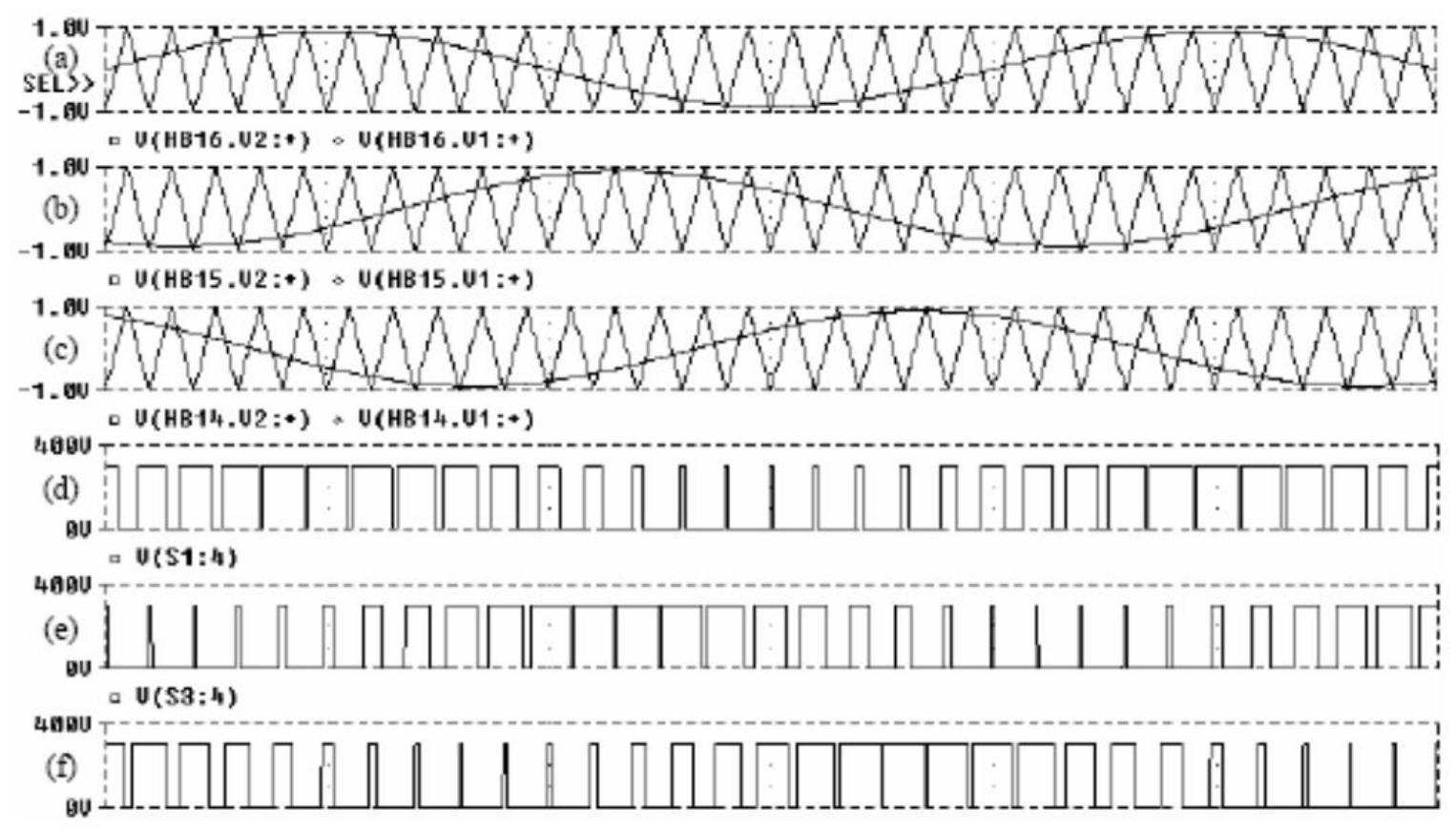

Figure 3.6: Three-phase SPWM waveforms [16].

The vector PWM (SVPWM) waveform is realized by the combination of different switching modes of the inverter. In a three-phase inverter, if 1 is defined as the positive half of the DC-bus voltage and 0 as the negative half (both are referred to 
the neutral point), there are eight switch states for the six power switches shown in Figure 3.7. Therefore eight voltage vectors (the active vectors $\vec{U}_{1} \sim \vec{U}_{6}$, and zero vectors $\vec{U}_{0}, \vec{U}_{7}$ ) can be correspondingly defined to form the vector space which is divided into six sectors shown in Figure 3.8.

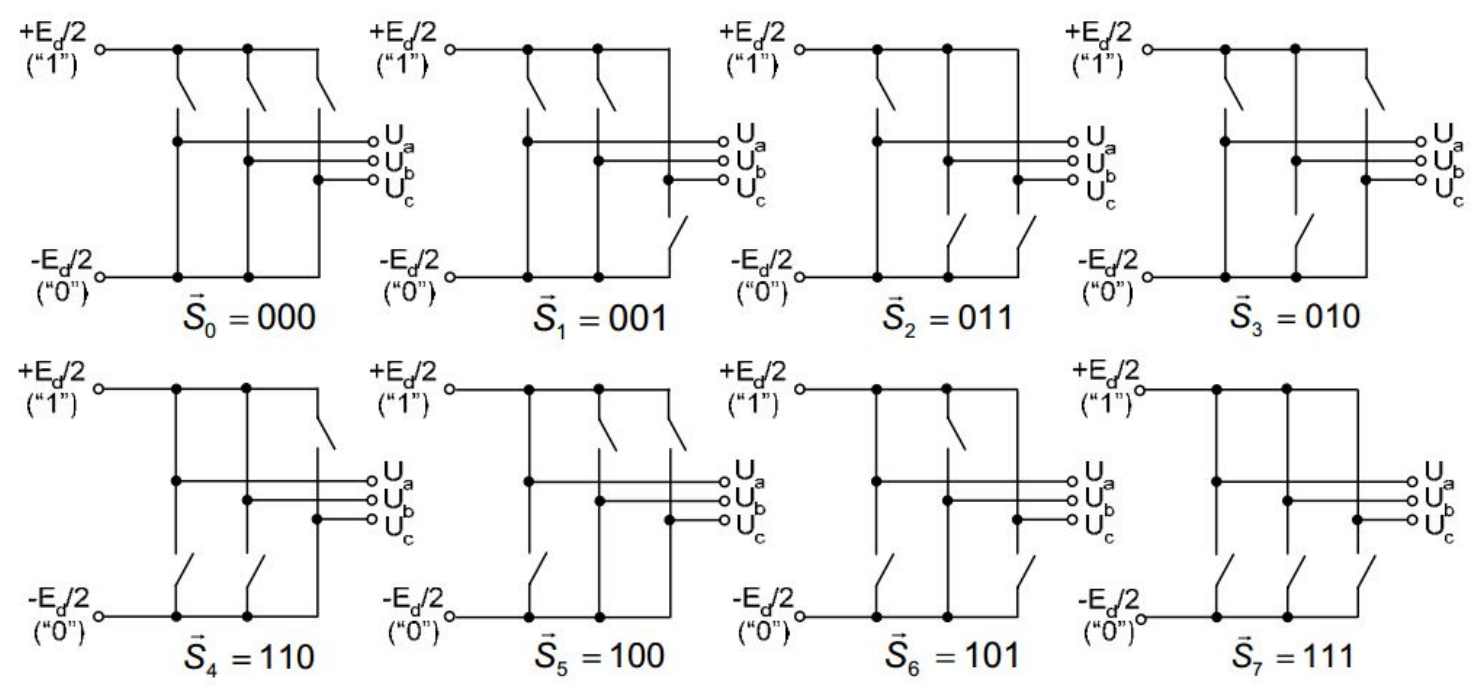

Figure 3.7: Eight switch states [16].

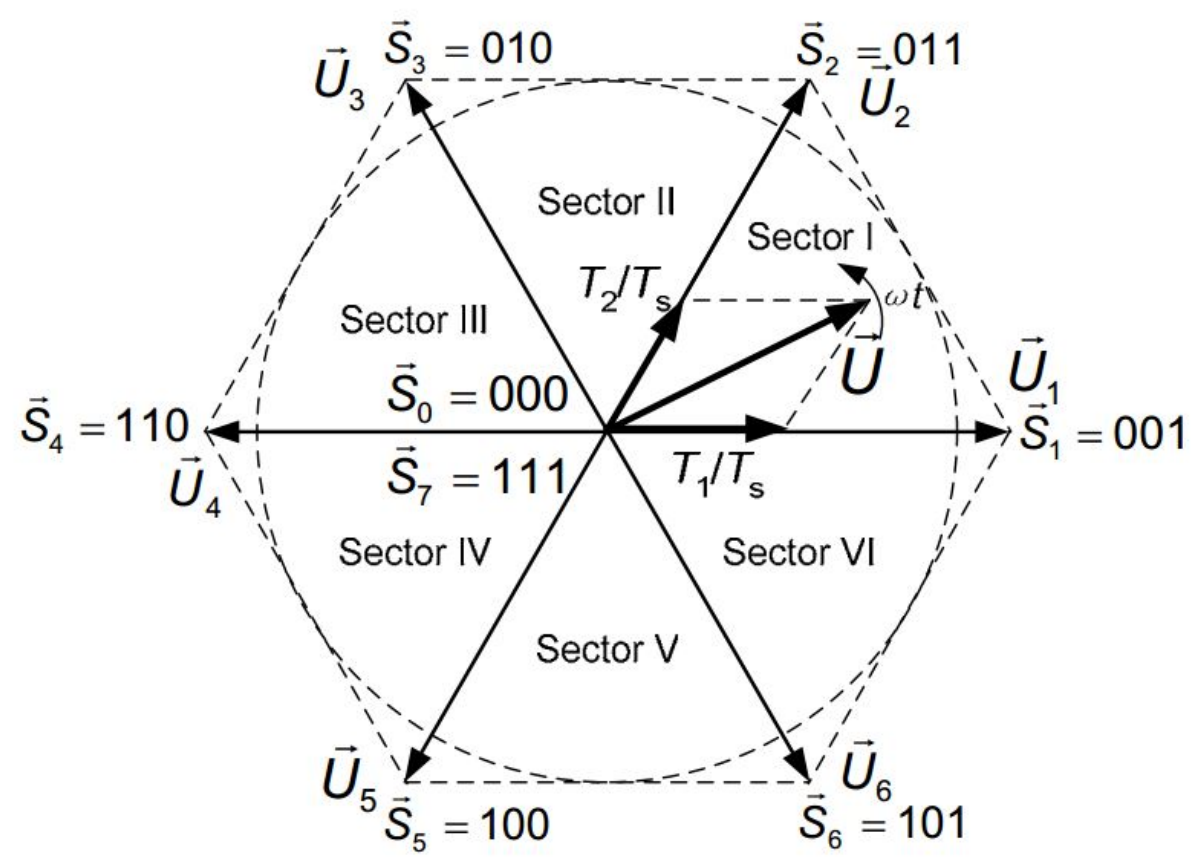

Figure 3.8: Voltage vector space [16]. 
Grid-Connecting Process Before discussing the current controller for the grid connected inverter system, the process before the inverter is connected to the grid should be introduced. Being one of the crucial technical aspects in the inverter control problem, the inverter system first should generate the voltage that has some related magnitude, same frequency and same phase as the grid and then can be connected to the grid. The grid-connecting process follows two steps:

Step 1 The inverter should first start working isolated from the grid and generate the three phase voltage that has the same magnitude as the grid side voltage by a transformer. The maximum tolerance error of the inverter output voltage is $10 \%$ of the rated voltage.

Step 2 With the inverter generating the same magnitude three phase voltage, the phase tracking method is used to give the closing signal [22], [23]. The basic principle is to connect the inverter to the grid at the moment when the inverter system output has the same frequency and same phase as the grid.

With above steps accomplished, the inverter system can be successfully connected to the grid. Figure 3.9 shows the block diagram of the control of the grid-connection process. In this thesis, we will be considering mainly about the current control problem for the grid-connected system, which is after this grid connection process is accomplished. 


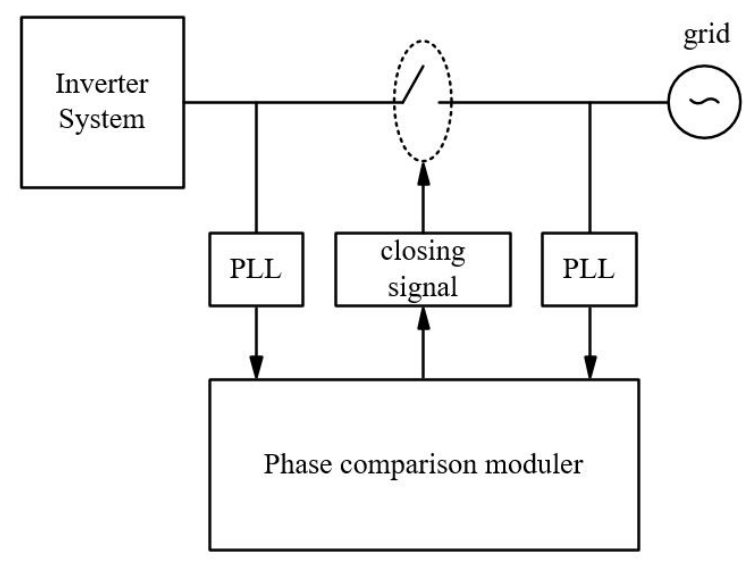

Figure 3.9: Scheme diagram for inverter-grid connecting process.

\subsection{State-of-the-Art of Inverter Control}

To solve the control problem for PV inverter systems, there are many methods in recent years. While most of the control methods use classical control design such as PI control, there are some adaptive methods as well as other advanced methods towards this problem.

Adaptive Droop Control Approach In [5] they come up with an adaptive droop control method to the single-phase voltage source inverters operating in both gridconnected and island modes. They build the droop controller to directly control the power of $P, Q$ the system where $P$ represents the active output power and $Q$ represents the reactive power, and adaptively estimate the system parameters (phase the signals, impedance in the circuit, frequency of the signals) from the output voltage and current of the inverter to achieve the adaptive control objective. This is a novel method for the inverter control problem for both grid-connected and island modes and this method directly controls the system power, which makes the structure of the control system more straight forward. With the adaptive estimates of the system 
parameters, an adaptive control scheme is developed. However, the limitation of this research is that this control method is proposed for the single phase inverter and the adaptive method to estimate the system parameters is not suitable for three-phase problems. Moreover, they did not consider the harmonic elimination problem since it is only a single-phase system, which cannot be ignored when we design a controller for the three-phase systems.

Other Adaptive Control Approach In [6], they give an adaptive design for the three-phase inverter system in island mode. This research builds up an adaptive control scheme using a state feedback adaptive controller (a structure similar to the one used in this thesis). This design is proved that all closed-loop signals bounded and desired tracking error goes to zero. The adaptive control problem in this problem is a good adaptive approach towards the three-phase inverter system. However, this design did not consider the grid-connected case, which is the main problem for the three-phase inverter system. Also, they did not consider the elimination of the possible higher order harmonics.

PI+PR Control Approach In [7], the study gives a control design for the threephase inverter system. They used three control loops: a power loop gets the reference signal for the voltage, and last the voltage loop gets the reference signal for the current, then the current control loop gets the desired control signal for the system, the duty cycle signal. The combination of PI control and PR control is used in the control loop for the current loop and voltage loop. This method can deal with both grid-connected and island modes and meets the requirement for the Total Harmonic Distortion (THD). However, this research did not use any novel method to overcome the drawbacks of those control methods. They do not have the ability to make the output current signal tracking a reference signal which might vary through time and 
this method may be too complicated for three phase systems since there will be three control loop for each phase. Compared to the previous two methods introduced above, the controller structure in this research is much more complicated than the previous two.

Open Technical Issues From the discussion above, we can see that though there are many research results to develop novel control techniques for the inverter system, few of them are aimed at dealing with the uncertainties for the inverter system, especially for the PV inverter system. Moreover, most researches to develop a control scheme that used the adaptive principle for the inverter system were aimed at one specific problem in the inverter system each time. In this thesis, a systematical study adaptive control is conducted for the PV inverter system.

\subsection{System Dynamic Equations}

In this section, the state-space equation for the inverter system is derived. Figure 3.10 shows the basic structure of a typical grid-connected three-phase photovoltaic inverter [26], [27].

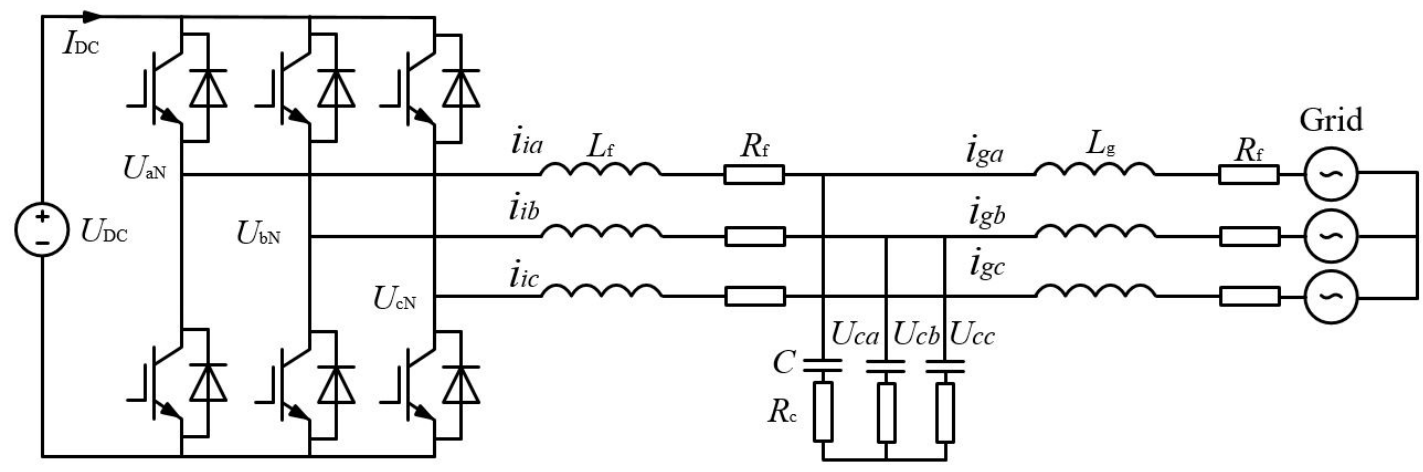

Figure 3.10: Structure of a three phase inverter 
where, $L_{f}$ represents the inductance of the inverter side, $L_{g}$ represents the inductance of the grid side, $C$ is the filter capacitor and $R_{f}, R_{g}$, and $R_{c}$ represent the Equivalent Series Resistance (ESR) of each part. $I_{P V} / U_{P V}$ is the input current/voltage for the inverter system, and $i_{i a, b, c}$ is the inverter side current and $i_{g a, b, c}$ is the grid side current. The control signal for the system $u(t)$ is the duty cycle of the PWM waveform, which was stated in Section 3.1. According to the Kirchhoff Current and Voltage laws, we can obtain the dynamic equations of the inverter under a stationary coordinate system as

$$
\begin{aligned}
\frac{\mathrm{d}}{\mathrm{d} t}\left[\begin{array}{l}
I_{i a} \\
I_{i b} \\
I_{i c}
\end{array}\right] & =\frac{u_{P V}}{\sqrt{3} L_{f}}\left[\begin{array}{l}
d_{a} \\
d_{b} \\
d_{c}
\end{array}\right]-\frac{R_{f}}{L_{f}}\left[\begin{array}{l}
I_{i a} \\
I_{i b} \\
I_{i c}
\end{array}\right]-\frac{R_{c}}{L_{f}}\left[\begin{array}{l}
I_{i a}-I_{g a} \\
I_{i b}-I_{g b} \\
I_{i c}-I_{g c}
\end{array}\right]-\frac{1}{L_{f}}\left[\begin{array}{l}
U_{a c} \\
U_{b c} \\
U_{c c}
\end{array}\right] \\
\frac{\mathrm{d}}{\mathrm{d} t}\left[\begin{array}{l}
I_{g a} \\
I_{g b} \\
I_{g c}
\end{array}\right] & =\frac{1}{L_{g}}\left[\begin{array}{l}
U_{a c} \\
U_{b c} \\
U_{c c}
\end{array}\right]+\frac{R_{g}}{L_{g}}\left[\begin{array}{l}
I_{i a} \\
I_{i b} \\
I_{i c}
\end{array}\right]+\frac{R_{c}}{L_{g}}\left[\begin{array}{l}
I_{i a}-I_{g a} \\
I_{i b}-I_{g b} \\
I_{i c}-I_{g c}
\end{array}\right]-\frac{1}{L_{g}}\left[\begin{array}{l}
U_{a N} \\
U_{b N} \\
U_{c N}
\end{array}\right] \\
\frac{\mathrm{d}}{\mathrm{d} t}\left[\begin{array}{c}
U_{a c} \\
U_{b c} \\
U_{c c}
\end{array}\right] & =-\frac{1}{C}\left[\begin{array}{l}
I_{i a}-I_{g a} \\
I_{i b}-I_{g b} \\
I_{i c}-I_{g c}
\end{array}\right],
\end{aligned}
$$

where $d_{a}, d_{b}, d_{c}$ are the duty cycles of the PWM waveform, that is, the control vector is $u(t)=\left[d_{a}, d_{b}, d_{c}\right]^{T}$.

Coordinate Transformation equation If we directly build the state space equation base on the equations above, the system will have nine states and three outputs, and we will need to calculate three reference signals for the system outputs. This will make the controller more difficult to design. Moreover, for the adaptive controller, more states will make the number of estimated parameters increase in an even faster rate. For the analysis of three-phase synchronous systems, people tend to use the direct-quadrature-zero $(D Q Z)$ transform to change the vectors in the a-b-c reference frame to the d-q-o reference frame [28], [29]. For our inverter system, since there exists no neutral line connection, the $\mathrm{Z}$ component is identically zero. The $D Q Z$ transformation matrix is 


$$
\begin{gathered}
T=\sqrt{\frac{2}{3}}\left[\begin{array}{ccc}
\cos (\omega t) & \cos \left(\omega t-\frac{2 \pi}{3}\right) & \cos \left(\omega t+\frac{2 \pi}{3}\right) \\
\cos (\omega t) & \cos \left(\omega t-\frac{2 \pi}{3}\right) & \cos \left(\omega t+\frac{2 \pi}{3}\right) \\
\frac{1}{\sqrt{2}} & \frac{1}{\sqrt{2}}
\end{array}\right], \\
{\left[\begin{array}{l}
x_{d} \\
x_{q} \\
x_{o}
\end{array}\right]=T\left[\begin{array}{l}
x_{a} \\
x_{b} \\
x_{c}
\end{array}\right] \Leftrightarrow\left[\begin{array}{l}
x_{a} \\
x_{b} \\
x_{c}
\end{array}\right]=T^{-1}\left[\begin{array}{l}
x_{d} \\
\left.x_{q}\right] \\
x_{o}
\end{array}\right]}
\end{gathered}
$$

where $T T^{T}=I$. Applying the $D Q Z$ Transformation above, we can change the three phase equations (3.1)-(3.3) from the a-b-c reference to the d-q-o reference frame (3.6)-(3.8). With the fact that there is no neutral line connection, the terms associates with the axis $o$ will become identically zero. As a result, we will obtain the new state equations with three less states. This will make our adaptive control design simpler.

$$
\begin{aligned}
\frac{\mathrm{d}}{\mathrm{d} t}\left[\begin{array}{l}
I_{i d} \\
I_{i q}
\end{array}\right] & =\frac{u_{P V}}{\sqrt{3} L_{f}}\left[\begin{array}{l}
d_{d} \\
d_{q}
\end{array}\right]-\frac{R_{f}}{L_{f}}\left[\begin{array}{l}
I_{i d} \\
I_{i q}
\end{array}\right]-\frac{R_{c}}{L_{f}}\left[\begin{array}{l}
I_{i d}-I_{g d} \\
I_{i q}-I_{g q}
\end{array}\right]-\frac{1}{L_{f}}\left[\begin{array}{c}
U_{P V} \\
U_{q c}
\end{array}\right]-\omega\left[\begin{array}{c}
-I_{q} \\
I_{d}
\end{array}\right] \\
\frac{\mathrm{d}}{\mathrm{d} t}\left[\begin{array}{c}
I_{g d} \\
I_{g q}
\end{array}\right] & =\frac{1}{L_{g}}\left[\begin{array}{c}
U_{P V} \\
U_{q c}
\end{array}\right]+\frac{R_{g}}{L_{g}}\left[\begin{array}{l}
I_{i d} \\
I_{i q}
\end{array}\right]+\frac{R_{c}}{L_{g}}\left[\begin{array}{c}
I_{i d}-I_{g d} \\
I_{i q}-I_{g q}
\end{array}\right]-\frac{1}{L_{g}}\left[\begin{array}{c}
U_{d N} \\
U_{q N}
\end{array}\right]-\omega\left[\begin{array}{c}
-I_{g q} \\
I_{g d}
\end{array}\right] \\
\frac{\mathrm{d}}{\mathrm{d} t}\left[\begin{array}{c}
U_{P V} \\
U_{q c}
\end{array}\right] & =-\frac{1}{C}\left[\begin{array}{c}
I_{i d}-I_{g d} \\
I_{i q}-I_{g q}
\end{array}\right]-\omega\left[\begin{array}{c}
-U_{q c} \\
U_{P V}
\end{array}\right] .
\end{aligned}
$$

State Space Form Dynamic Equation By Rewriting (3.6)-(3.8) in the state space form, we have

$$
\dot{x}(t)=A x(t)+B u(t)+B_{d} \delta(t), y(t)=C x(t),
$$


where

$$
\begin{aligned}
& x=\left[\begin{array}{llllll}
I_{i d} & I_{i q} & I_{g d} & I_{g q} & U_{P V} & U_{q c}
\end{array}\right]^{T}, \quad u=\left[\begin{array}{ll}
d_{d} & d_{q}
\end{array}\right]^{T}, \\
& A=\left[\begin{array}{cccccc}
-\frac{R_{f}+R_{c}}{L_{f}} & \omega & \frac{R_{c}}{L_{f}} & 0 & -\frac{1}{L_{f}} & 0 \\
-\omega & -\frac{R_{f}+R_{c}}{L_{f}} & 0 & \frac{R_{c}}{L_{f}} & 0 & -\frac{1}{L_{f}} \\
\frac{R_{c}+R_{g}}{L_{g}} & 0 & -\frac{R_{c}}{L_{g}} & \omega & \frac{1}{L_{g}} & 0 \\
0 & \frac{R_{c}+R_{g}}{L_{g}} & -\omega & -\frac{R_{c}}{L_{g}} & 0 & \frac{1}{L_{g}} \\
\frac{1}{C} & 0 & -\frac{1}{C} & 0 & 0 & \omega \\
0 & \frac{1}{C} & 0 & -\frac{1}{C} & -\omega & 0
\end{array}\right], \\
& B=\left[\begin{array}{cccccc}
\frac{U_{P V}}{\sqrt{3} L_{f}} & 0 & 0 & 0 & 0 & 0 \\
0 & \frac{U_{P V}}{\sqrt{3} L_{f}} & 0 & 0 & 0 & 0
\end{array}\right]^{T}, B_{d}=\left[\begin{array}{cccccc}
0 & 0 & -\frac{1}{L_{g}} & 0 & 0 & 0 \\
0 & 0 & 0 & -\frac{1}{L_{g}} & 0 & 0
\end{array}\right]^{T}, \\
& C=\left[\begin{array}{cccccc}
0 & 0 & 1 & 0 & 0 & 0 \\
0 & 0 & 0 & 1 & 0 & 0
\end{array}\right], \quad d=\left[\begin{array}{ll}
U_{d N} & U_{q N}
\end{array}\right]^{T} \text {. }
\end{aligned}
$$

where $u(t)=\left[d_{d}, d_{q}\right]^{T}$ is the control signal, $d_{d}$ and $d_{q}$ are the duty cycles in the d-q axis, and $\delta$ is the grid side voltage, in these state-space equations we consider it to be the disturbance of the system.

Remark 3.1. Notice that this model is derived for the three-phase grid-connected PV inverter. For the three phase PV inverter that works without grid influence, the state space form dynamic equation is:

$$
\dot{x}(t)=A x(t)+B u(t), y(t)=C x(t),
$$

where $A, B, C$ has the same value as the system stated in (3.9). This is the same as the inverter system (3.9) with disturbance term $d=0$ indicating that the grid side 
voltage is zero, that is, the inverter is isolated from the grid.

\subsection{Control Problem Formulation}

Based on the requirements for the inverter system, the control objective is formed in this section.

Control Objectives To deal with the system uncertainties which are discussed in Chapter 1 and characterized by unknown parameters in the matrices $A, B, C$ and $B_{d}$, we need to design an adaptive controller for the system (3.9) or (3.14), to achieve the following objectives:

(i) The adaptive control signal $\mathrm{u}(\mathrm{t})$ for the system (3.9) makes $\mathrm{y}(\mathrm{t})$ track $y_{m}(t)$, where

$$
\begin{aligned}
y(t) & =\left[\begin{array}{ll}
I_{g d}(t) & I_{g q}(t)
\end{array}\right]^{T} \\
y_{m}(t) & =\left[\begin{array}{ll}
I_{g d}^{*}(t) & 0
\end{array}\right]^{T} ;
\end{aligned}
$$

with the value of $I_{g d}^{*}$ being obtained from the inverter requirement which is discussed in the later part of this section.

(ii) The adaptive control signal $\mathrm{u}(\mathrm{t})$ should have the ability to reject the disturbance $\delta(t)$ in $(3.11)$, where $\delta(t)$ has the form

$$
\delta(t)=\left[\begin{array}{c}
U_{d N}(t) \\
U_{q N}(t)
\end{array}\right]=\left[\begin{array}{c}
U_{d 1 N}+U_{d 6 c N} \cos (6 \omega t)+U_{d 6 s N} \sin (6 \omega t)+\ldots \\
U_{q 6 c N} \cos (6 \omega t)+U_{q 6 s N} \sin (6 \omega t)+\ldots
\end{array}\right]
$$

This disturbance represents the grid side voltage and its harmonic components which will have some negative effects for the inverter system. 
Inverter System Requirements These control objectives are consistent with the goals that a PV inverter needs to achieve. A good PV grid-connected inverter needs to meet the following requirements:

(1) The output current should have the same frequency and same phase with the grid side voltage. This function can be achieved by obtaining the frequency and phase of the system by the phase lock loop (PLL) module, where after the desired output signals are obtained, apply $i_{d} i_{q}$ back to the inverse online DQZ transform action. Then, we can ensure the input-output signals to have same frequency and phase.

(2) The system should have some robustness to the system uncertainties and disturbance. This means that the system should be able to work under some uncertainties. For instance, the system should be able to handle some changes of the system parameters, or the change of the grid side voltage.

(3) The quality of the output current should meet some requirement. The IEEE 1527 standard allows a limit of 5\% for current total harmonic distortion (THD) factor with individual limits of $4 \%$ for each add harmonics from $3^{\text {rd }}$ to $9^{\text {th }}$ and $2 \%$ for $11^{\text {th }}$ to $15^{\text {th }}$ while the European standard IEC61727 suggests similar. The harmonics mainly come from the grid side. We can write the grid side voltage with the harmonics from the grid as

$$
\left[\begin{array}{c}
U_{a N}(t) \\
U_{b N}(t) \\
U_{c N}(t)
\end{array}\right]=\left[\begin{array}{c}
U_{a 1 N} \cos (\omega t)-U_{a 5 N} \cos (5 \omega t)+U_{a 7 N} \cos (7 \omega t)+\ldots \\
U_{b 1 N} \cos \left(\omega t-\frac{2 \pi}{3}\right)-U_{b 5 N} \cos \left(5\left(\omega t-\frac{2 \pi}{3}\right)\right)+U_{b 7 N} \cos \left(7\left(\omega t-\frac{2 \pi}{3}\right)\right)+\ldots \\
U_{c 1 N} \cos \left(\omega t+\frac{2 \pi}{3}\right)-U_{c 5 N} \cos \left(5\left(\omega t+\frac{2 \pi}{3}\right)\right)+U_{c 7 N} \cos \left(7\left(\omega t+\frac{2 \pi}{3}\right)\right)+\ldots
\end{array}\right] .
$$

The higher order of harmonics such as $11^{\text {th }}, 13^{\text {th }}$ and $17^{\text {th }}$ and $19^{\text {th }} \ldots$, can be 
eliminated by the LCL filter of the system. However, as for the lower-order harmonics, like $5^{\text {th }}$ and $7^{\text {th }}$, they cannot be eliminated just by only using the filters and will influence the system behavior. From the system state equation in (3.9), $\delta(t)$ represents the grid side disturbance. By applying the DQZ transform, the harmonics in the above equation can be rewritten in the rotating coordinate form, which is the grid side disturbance:

$$
\delta(t)=\left[\begin{array}{c}
U_{d N}(t) \\
U_{q N}(t)
\end{array}\right]=\left[\begin{array}{c}
U_{d 1 N}+U_{d 6 c N} \cos (6 \omega t)+U_{d 6 s N} \sin (6 \omega t)+\ldots \\
U_{q 6 c N} \cos (6 \omega t)+U_{q 6 s N} \sin (6 \omega t)+\ldots
\end{array}\right]
$$

From the control point of view, elimination of the harmonics of the output current is equivalent to rejection of the grid side disturbance $\delta(t)$.

(4) The output only contains active power but no reactive power. The output power of the inverter is

$$
\begin{aligned}
P & =\frac{2}{3}\left(I_{g d} U_{d N}+I_{g q} U_{q N}\right) \\
Q & =\frac{2}{3}\left(I_{g q} U_{d N}+I_{g d} U_{q N}\right) .
\end{aligned}
$$

Since our primary concern is to make the output current smooth, under threephase balance condition, we can consider all the higher order terms in (3.11) going to zero when we calculate the output power of the system. With this assumption, (3.12) and (3.13) can be rewritten as

$$
\begin{aligned}
& P=\frac{2}{3}\left(I_{g d} U_{d N}+I_{g q} U_{q N}\right)=\frac{2}{3} I_{g d} U_{d 1 N} \\
& Q=\frac{2}{3}\left(I_{g q} U_{d N}+I_{g d} U_{q N}\right)=\frac{2}{3} I_{g q} U_{d 1 N} .
\end{aligned}
$$

From the above equations, $I_{g q}=0$ is needed to achieve no reactive power. From 
the control point of view, no reactive power is equivalent to letting $I_{g q}$ to track zero. Also, we want the system to work at the maximum power point, with the MPPT module which we introduced in 2.1, where the desired active power $P^{*}(t)$ is obtained. Thus, the reference current signal for $I_{g d}$ is

$$
I_{g d}^{*}(t)=\frac{2 P^{*}(t)}{3 U_{d 1 N}}
$$

As a result, the control objective stated above meets all the requirements for the three phase inverter system. With this control objective in mind, we can start the adaptive control design. 


\section{Chapter 4}

\section{State Feedback Adaptive Control}

\section{Scheme}

In this chapter, a state feedback output tracking MRAC scheme is derived to control the main circuit of the three-phase PV inverter. The control scheme is first designed for the case where the PV inverter works without the grid side influence, and then designed for the general case where the PV inverter works with the gridconnection. Simulation results are shown at the end of the chapter to verify the control designs.

\subsection{Problem Formulation}

Consider the linear model for the three phase PV inverter system

$$
\dot{x}(t)=A x(t)+B u(t)+B_{d} \delta(t), y(t)=C x(t),
$$

where $A \in R^{6 \times 6}, B \in R^{6 \times 2}, B_{d} \in R^{6 \times 2}$, and $C \in R^{2 \times 6}$ are unknown constant matrices, with $x(t) \in R^{6}, y(t) \in R^{2}$ being available for measurement. They are 
obtained from Section 3.3. In this model, $\delta(t) \in R^{2}$ is an unmatched disturbance of the system because $B$ and $B_{d}$ are linearly independent, which can be written in the form

$$
\begin{aligned}
\delta(t) & =\left[\begin{array}{c}
\delta_{10}+\sum_{k=1}^{6} \delta_{1 k} f_{1 k}(t) \\
\delta_{20}+\sum_{k=1}^{6} \delta_{2 k} f_{2 k}(t)
\end{array}\right] \\
& =\left[\begin{array}{c}
U_{d N}(t) \\
U_{q N}(t)
\end{array}\right]=\left[\begin{array}{c}
U_{d 1 N}+U_{d 6 c N} \cos (6 \omega t)+U_{d 6 s N} \sin (6 \omega t)+\ldots \\
U_{q 6 c N} \cos (6 \omega t)+U_{q 6 s N} \sin (6 \omega t)+\ldots
\end{array}\right] .
\end{aligned}
$$

Notice that $B_{d} \neq \alpha B$ for any $\alpha \in R^{2 \times 2}$, for the obtained form of $B$ and $B_{d}$ of the three phase grid-connected PV inverter system. The physical meaning of the state space equation (4.1) is that the dynamics of the inverter system states include the current from the inverter side, the current from the grid side and the voltage of the filter capacitor. The control input signal $u(t)$ is the duty cycle that is applied to the PWM wave generator. The disturbance $\delta(t)$ represents the grid side influence for the inverter system. This grid side influence can either be the influence of the grid side fundamental frequency voltage or the grid side polluted voltage which contains some harmonic components.

The control objective for this adaptive control problem with disturbance compensation is to design an adaptive controller to generate $u(t)$ to guarantee the closed-loop signal boundedness and to make the output signal $y(t)$ to track a chosen reference signal generated from a reference model:

$$
y_{m}(t)=W_{m}(s)[r](t)=\left[\begin{array}{c}
I_{g d}^{*} \\
I_{g q}^{*}
\end{array}\right] \in R^{2}, \quad t \geq 0
$$

where $W_{m}(s) \in R^{2 \times 2}$ is a stable transfer function matrix, and $r(t) \in R^{2}$ is the 
reference input signal which can be defined when $y_{m}(t)$ is chosen. For the control problem of three phase PV inverter system, we can always obtain the desired reference

signal $I_{g d}^{*}$ and $I_{g q}^{*}$, where $I_{g q}^{*}$ is always set to be zero, and from the discussion in Section 3.4 when the grid side is under three phase balance condition $I_{g d}^{*}=\frac{2 P^{*}(t)}{3 U_{d 1 N}}$, where $P^{*}$ can be obtained from the MPPT module. Note that when the grid side is operating under three phase unbalance situation, the state-of-art research can still obtain the desired value of $I_{g d}^{*}$ and $I_{g q}^{*}$ for this control problem.

\subsection{Basic Design Conditions}

Before beginning the adaptive control design, we need to show that the three phase PV inverter system stated in (4.1) meets the following design conditions for multivariable adaptive disturbance rejection design.

Basic Assumptions for MRAC Before introducing the design conditions, the definitions of the high-frequency gain matrix and interactor matrix are introduced.

Lemma 4.1. For any $M \times M$ strictly proper rational full-rank transfer matrix, there exists a lower-triangular polynomial matrix $\xi_{m}(s)$, defined as the modified left interactor matrix of $G(s)$, has the form

$$
\xi_{m}(s)=\left[\begin{array}{ccccc}
d_{1}(s) & 0 & \ldots & \ldots & 0 \\
h_{21}^{m}(s) & d_{2}(s) & 0 & \ldots & 0 \\
\vdots & \vdots & \vdots & \vdots & \vdots \\
h_{M 1}^{m}(s) & h_{M 2}^{m}(s) & \ldots & h_{M M-1}^{m}(s) & d_{M}(s)
\end{array}\right]
$$

where $h_{i j}^{m}(s), j=1,2, \ldots, M-1, i=2, \ldots, M$, are some polynomials and $d_{i}(s)$ are any chosen monic stable polynomials such that the high-frequency gain matrix of $G(s)$ 
defined as $K_{p}=\lim _{s \rightarrow \infty} \xi_{m}(s) G(s)$ is finite and nonsingular [32].

To meet the control objective, we need to assume

Assumption 1 All zeros of $G(s)=C(s I-A)^{-1} B$ are stable, and (A,B,C) is stabilizable and detectable.

Assumption $2 G(s)$ has a diagonal interactor matrix $\xi_{m}=\operatorname{diag}\left\{d_{1}(s), d_{2}(s)\right\}$ where, $d_{i}(s)=s^{\rho_{i}}+a_{i \rho_{i}-1}^{*} s^{\rho_{i}-1}+\cdots+a_{i 1}^{*} s+a_{i 0}^{*}, i=1,2$ are stable monic polynomials such that the high-frequency gain matrix

$$
K_{p}=\lim _{s \rightarrow \infty} \xi_{m}(s) G(s)=\left[\begin{array}{l}
C_{1} A^{\rho_{1}-1} B \\
C_{2} A^{\rho_{2}-1} B
\end{array}\right] \in R^{2 * 2}
$$

is finite and nonsingular, where, $\rho_{i}, i=1,2$, are the relative degrees of $\left(C_{i}, A, B\right)$.

To verify these assumptions for the PV inverter system, from the definition of $(\mathrm{A}, \mathrm{B}, \mathrm{C})$ in Section 3.3, by using Matlab we can prove that the controllability matrix $S_{n}$ and observability matrix $L_{n}$ are such that

$$
\begin{aligned}
S_{n} & =\left[\begin{array}{lllll}
B & A B & A^{2} B & \ldots & A^{5} B
\end{array}\right], \operatorname{rank}\left(S_{n}\right)=6 \\
L_{n} & =\left[\begin{array}{lllll}
C^{T} & (C A)^{T} & \left(C A^{2}\right)^{T} & \ldots & \left(C A^{5}\right)^{T}
\end{array}\right]^{T}, \operatorname{rank}\left(L_{n}\right)=6 .
\end{aligned}
$$

This means that $(\mathrm{A}, \mathrm{B}, \mathrm{C})$ for the three phase $\mathrm{PV}$ inverter system is controllable and observable, which means that $(\mathrm{A}, \mathrm{B}, \mathrm{C})$ is stabilizable and detectable.

For the system zeros, it is hard to obtain a generic solution to prove that all system zeros are stable due to the high order matrices of this inverter system. As a result, a set of system parameters is chosen for the inverter system (A,B,C). Table 4.1 in Section 4.5 gives a set of system parameters. By applying this set of parameter to the system, 
we can verify that for this system the zeros are $(-5.56+j 0.0031) \times 10^{5},(-5.56-$ j0.0031) $\times 10^{5}$, they are indeed stable. Thus the system satisfies Assumption 1 .

As for Assumption 2, we can verify that for the inverter system $(A, B, C)$ which is stated in Section 3.3, it follows that

$$
\begin{gathered}
C B=\left[\begin{array}{ll}
0 & 0 \\
0 & 0
\end{array}\right] \\
C A B=\left[\begin{array}{cc}
\frac{\sqrt{3} * U_{P V}\left(R_{c}+R_{g}\right)}{3 L_{f} L_{g}} & 0 \\
0 & \frac{\sqrt{3} * U_{P V}\left(R_{c}+R_{g}\right)}{3 L_{f} L_{g}}
\end{array}\right] .
\end{gathered}
$$

Since all the system parameters are nonzero, the above equation means that the three phase PV inverter system has relative degrees $\rho_{i}=2, i=1,2$, and $K_{p}=C A B$ is a diagonal matrix with positive diagonal elements.

With these verifications above, we can obtain the following proposition for the three-phase inverter system.

Proposition 4.1. For the three phase inverter system with LCL filter stated in (4.1), the high-frequency gain matrix is

$$
K_{p}=\lim _{s \rightarrow \infty} \xi_{m}(s) G(s)=C A B=\left[\begin{array}{cc}
\frac{\sqrt{3} * U_{P V}\left(R_{c}+R_{g}\right)}{3 L_{f} L_{g}} & 0 \\
0 & \frac{\sqrt{3} * U_{P V}\left(R_{c}+R_{g}\right)}{3 L_{f} L_{g}}
\end{array}\right]
$$

and $G(s)$ has a diagonal interactor matrix $\xi_{m}=\operatorname{diag}\left\{d_{1}(s), d_{2}(s)\right\}$, where $d_{i}(s)=$ $s^{2}+a_{i 1}^{*} s+a_{i 0}^{*}$ are stable monic polynomials.

This result can be used in the control design for three-phase inverter systems. 


\subsection{Adaptive Control for Inverters without Dis- turbance}

Before working on the case with disturbance, we first consider the control design for the three phase PV inverter which works without grid influence, that is, isolated from the grid. With no grid connection, there is no disturbance $\delta(t)$ in the system, and the system (4.1) becomes

$$
\dot{x}(t)=A x(t)+B u(t), y(t)=C x(t)
$$

where $A \in R^{6 \times 6}, B \in R^{6 \times 2}$, and $C \in R^{2 \times 6}$ are unknown constant matrices, with $x(t) \in R^{6}, y(t) \in R^{2}$ available for measurement. The physical meaning of this model is, that the inverter system works isolated from the grid, which can be considered as the operation situation before the inverter is connected to the grid. Solving this control problem is a good start before working on the grid connected inverter system.

\subsubsection{Nominal Control Design}

Before starting the adaptive control design, first we will consider the case where all the system parameters are known, that is, the state feedback output tracking model reference control, to develop the controller structure for adaptive control.

Nominal Controller With the knowledge of system parameters, the nominal state feedback output tracking controller is

$$
u(t)=K_{1}^{*} x(t)+K_{2}^{*} r(t),
$$


where $K_{1}^{*} \in R^{2 \times 6}$ and $K_{2}^{*} \in R^{2 \times 2}$ are designed for output tracking.

For the three phase PV inverter system under Assumption 1, consider the relative degrees $\rho_{i}$ for the system $\left(C_{i}, A, B\right)$. With the form of the system shown in Section 3.2, we have $\rho_{i}=2, i=1,2$. The system equations are

$$
\dot{x}(t)=A x(t)+B u(t), y_{i}(t)=C_{i} x(t), y(t)=\left[\begin{array}{c}
y_{1}(t) \\
y_{2}(t)
\end{array}\right]
$$

and the $2^{\text {th }}$ order derivative of $y_{i}(t)$ is

$$
y_{i}^{(2)}(t)=C_{i} A^{2} x(t)+C_{i} A B u(t)=C_{i} A^{2} x(t)+K_{p i} u(t),
$$

where $K_{p i}$ is given in (4.6), so that $K_{p}=\left[K_{p 1}, K_{p 2}\right]^{T}$, is an inverterable matrix. Choose the control law as

$$
u(t)=K_{P}^{-1} v(t), v(t)=\left[\begin{array}{ll}
v_{1}(t) & v_{2}(t)
\end{array}\right]^{T}
$$

Then (4.15) becomes

$$
y_{i}^{(2)}(t)=C_{i} A^{2} x(t)+v_{i}(t)
$$

With the choice of

$$
v_{i}(t)=-C A^{2} x(t)-a_{i 1}^{*} \dot{y}_{i}-a_{i 0}^{*} y(t)+r_{i}(t),
$$

we have from (4.17) that

$$
y^{(2)}(t)+a_{i 1}^{*} \dot{y}_{i}+a_{i 0}^{*} y(t)=r_{i}(t)
$$


which means $y_{i}(s)=\frac{1}{d_{i}(s)} r_{i}(s)$, that is,

$$
y(t)=W_{m}(s) r(t)
$$

From discussion above, with the fact that $\rho_{i}=2$ known, the signal $\mathrm{v}(\mathrm{t})$ can be expressd as

$$
v(t)=K_{0} x(t)+r(t)
$$

where $K_{0}=\left[k_{01}^{T}, k_{02}^{T}\right]$ with

$$
k_{0 i}^{T}=-C_{i} A^{2}-a_{i 1}^{*} C_{i} A-a_{i 0}^{*} C_{i}, i=1,2
$$

Comparing with the definition of $u(t)$ in (4.13), we have

$$
u(t)=K_{p}^{-1}\left(K_{0} x(t)+r(t)\right)=K_{1}^{*} x(t)+K_{2}^{*} r(t)
$$

which leads to

$$
K_{1}^{*}=K_{p}^{-1} K_{0}, K_{2}^{*}=K_{p}^{-1}
$$

From the derivation above, we show that there exists a state feedback control law to achieve the desired output tracking:

$$
y(s)=C\left(s I-A-B K_{1}^{* T}\right)^{-1} B K_{2}^{* T} r(s)=W_{m}(s) r(s) .
$$

Matching Property of $K_{1}^{*}$ and $K_{2}^{*}$ From (4.25), we can find the matching condition to find $K_{1}^{*}, K_{2}^{*}$

$$
C\left(s I-A-B K_{1}^{* T}\right)^{-1} B K_{2}^{* T}=W_{m}(s) .
$$


With the derivation above, we can propose the following proposition

Proposition 4.2. There exist $K_{1}^{*} \in R^{6 \times 2}$ and $K_{2}^{*} \in R^{2 \times 2}$ such that the matching equation (4.26) holds if $\xi_{m}(s)$ is the modified interactor matrix of $G(s)=C(s I-$ $A)^{-1} B$.

With parameters satisfying the matching equation (4.26) above, in view of (4.25), we have that the nominal controller (4.23) ensures that all signals in the closed-loop system are bounded and $\lim _{t \rightarrow \infty}\left(y(t)-y_{m}(t)\right)=0$

\subsubsection{Adaptive Control Design}

With the nonminal controller obtained, in this subsection the state feedback output tracking model reference control design is developed for the inverter with unknown parameters, working without the grid side influence.

Controller Structure The adaptive version of the nonminal controller (4.13) is

$$
u(t)=K_{1}^{T}(t) x(t)+K_{2}(t) r(t)
$$

where $K_{1} \in R^{6 \times 2}$ and $K_{2} \in R^{2 \times 2}$ are estimates of $K_{1}^{*}$ and $K_{2}^{*}$.

Tracking Error Equation Apply the adaptive controller to the plant (4.12), we can obtain the following closed-loop system

$$
\begin{aligned}
& \dot{x}(t)=\left(A+B K_{1}^{* T}\right) x(t)+B K_{2}^{*} r(t)+B\left(K_{1}^{T}-K_{1}^{* T}\right) x(t)+B\left(K_{2}-K_{2}^{*}\right) r(t) \\
& y(t)=C x(t) .
\end{aligned}
$$

From the definition of the reference signal (4.4), the parameter matching condition (4.26), and this obtained closed-loop system, we can derive the output tracking error 
equation as

$$
\begin{aligned}
e(t) & =y(t)-y_{m}(t) \\
& =W_{m}(s) K_{2}^{*-1}\left[\tilde{\Theta}^{T} \omega\right](t)+C e^{\left(A+B K_{1}^{* T}\right) t} x(t),
\end{aligned}
$$

where

$$
\begin{aligned}
\Theta^{* T} & =\left[K_{1}^{* T}, K_{2}^{*}\right] \\
\omega(t) & =\left[x^{T}(t), r^{T}(t)\right]^{T} \\
\Theta^{T}(t) & =\left[K_{1}^{T}(t), K_{2}(t)\right] \\
\tilde{\Theta}(t) & =\Theta(t)-\Theta^{*}
\end{aligned}
$$

The term $C e^{\left(A+B K_{1}^{* T}\right) t} x(t)$ converge to zero exponentially fast due to the stability of $A+B K_{1}^{* T}$. As a result we can just write the output tracking error as

$$
e(t)=y(t)-y_{m}(t)=W_{m}(s) K_{2}^{*-1}\left[\tilde{\Theta}^{T} \omega\right](t)
$$

Estimation Error Equation To develop adaptive law to update controller parameter $\Theta(t)$, we need further define the estimation error. First choose the filter $h(s)=\frac{1}{f(s)}$, where $f(s)$ is a stable polynomial $f(s)=s^{2}+f_{1} s+f_{0}$ of degree 2 . Then we can define the estimation error as

$$
\varepsilon(t)=\xi_{m}(s) h(s)\left[y-y_{m}\right](t)+K_{p}(t) \xi(t)=\xi_{m}(s) h(s)[e](t)+K_{p}(t) \xi(t) .
$$


Here $K_{p}(t)$ is the estimate of the high-frequency gain matrix $K_{p}^{*}$ in Assumption 2, $\xi_{m}(t)$ is the diagonal interactor matrix $\xi_{m}(s)=\operatorname{diag}\left\{d_{1}(s), d_{2}(s)\right\}$, and

$$
\begin{aligned}
& \xi(t)=\Theta^{T}(t) \zeta(t)-h(s)[u](t) \\
& \zeta(t)=h(s)[\omega](t)
\end{aligned}
$$

With the output tracking error equation (4.36), we can rewrite the estimation error as

$$
\varepsilon(t)=K_{p}^{*} \tilde{\Theta}(t) \zeta(t)+\tilde{K}_{p}(t) \xi(t), \tilde{K}_{p}(t)=K_{p}(t)-K_{p}^{*}
$$

Adaptive Laws Based on the estimation error equation (4.40), the adaptive parameter update laws are chosen as

$$
\begin{aligned}
& \dot{\Theta}^{T}(t)=-\frac{\Gamma_{1} \varepsilon(t) \zeta^{T}(t)}{m^{2}(t)} \\
& \dot{K}_{p}(t)=-\frac{\Gamma_{2} \varepsilon(t) \xi^{T}(t)}{m^{2}(t)}
\end{aligned}
$$

where $\Gamma_{i}=\Gamma_{i}^{T}>0 i=1,2, m^{2}(t)=1+\zeta^{T}(t) \zeta(t)+\xi^{T}(t) \xi(t)$.

Stability Analysis With the adaptive control design above, we can prove that the following lemma is true for our parameter adaptive law.

Lemma 4.2. The adaptive laws (4.41)-(4.42) guarantee that

(i) $\Theta(t) \in L^{\infty}, K_{p}(t) \in L^{\infty}, \frac{\varepsilon(t)}{m(t)} \in L^{2} \cap L^{\infty}$;

(ii) $\dot{\Theta}(t) \in L^{\infty} \cap L^{2}, \dot{K}_{p}(t) \in L^{\infty} \cap L^{2}$.

Proof. Choose the positive definite function

$$
V=\frac{1}{2}\left(\operatorname{tr}\left[\tilde{K}_{p}^{T} \Gamma_{2}^{-1} \tilde{K}_{p}\right]+\operatorname{tr}\left[\tilde{\Theta}^{T} \Gamma_{1}^{-1} \tilde{\Theta}\right]\right)
$$


With the adaptive laws (4.41), (4.42), the time derivative of $\mathrm{V}$ can be derived as

$$
\dot{V}=-\frac{\varepsilon^{T}(t) \varepsilon(t)}{m^{2}(t)} \leq 0
$$

Thus, we can conclude that $\Theta(t) \in L^{\infty}, K_{p}(t) \in L^{\infty}, \frac{\varepsilon(t)}{m(t)} \in L^{2} \cap L^{\infty}, \dot{\Theta}(t) \in L^{\infty} \cap L^{2}$, $\dot{K}_{p}(t) \in L^{\infty} \cap L^{2}$

Based on this lemma, one can prove that the closed-loop system has the following desired properties.

Theorem 4.1. For the plant (4.12) with uncertainties for the system parameter under Assumption 1 and 2 and the reference model (4.4), the adaptive controller (4.27) with the adaptive update laws (4.41), (4.42) can guarantee all signals in the closedloop system are bounded and the output tracking error $e(t)=y(t)-y_{m}(t)$ satisfies $\lim _{t \rightarrow \infty} e(t)=0$.

With this theorem, the performance of the adaptive controller designed in this section can achieve output tracking of the given signal $y_{m}$.

\subsection{Adaptive Control for Grid-Connected PV In- verter}

For the PV inverter that works grid-connected, we consider the grid side voltage as the disturbance for our system. As a result, the three-phase grid connected PV inverter control problem is the adaptive disturbance rejection control problem. In this section, the state feedback output tracking MRAC control scheme with adaptive disturbance rejection ability is designed in comparison with the nominal state feedback design with disturbance rejection. The state-space equation for the inverter 
system with grid-connection is

$$
\dot{x}=A x+B u+B_{d} d, y=C x
$$

where $A, B, C$ and $B_{d}$ are the same as stated in Section 3.3.

\subsubsection{Nominal Control Design}

Similar to the previous section, before starting the adaptive control design, a nominal model reference controller is designed first.

Nominal Controller With the knowledge of system parameters and disturbance parameters, the nominal state feedback output tracking controller is

$$
u(t)=K_{1}^{*} x(t)+K_{2}^{*} r(t)+K_{3}^{*}(t),
$$

where $K_{1}^{*} \in R^{2 \times 6}$ and $K_{2}^{*} \in R^{2 \times 2}$ are designed for output tracking and $K_{3}^{*}(t) \in R^{2}$ is for canceling the effect of the disturbance term $\delta(t)$ in the system.

For the three phase PV inverter system under Assumptions 1 and 2, consider the relative degree $v_{i}$ for the disturbance system $\left(C_{i}, A, B_{d}\right)$ and the relative degree $\rho_{i}$ for the control system $\left(C_{i}, A, B\right)$. With the form of the system shown in Section 2.2, we have $\rho_{i}=2, i=1,2$, and $v_{i}=1, i=1,2, \rho_{i}>v_{i}$.

The system equations are

$$
\dot{x}(t)=A x(t)+B u(t)+B_{d} \delta(t), y_{i}(t)=C_{i} x(t), y(t)=\left[\begin{array}{l}
y_{1}(t) \\
y_{2}(t)
\end{array}\right]
$$


The $2^{\text {nd }}$ order derivative of $y_{i}(t)$ is

$$
y_{i}^{(2)}(t)=C_{i} A^{2} x(t)+C_{i} A B u(t)+C_{i} A B_{d} \delta(t)+C_{i} B_{d} \dot{\delta}(t)
$$

For $K_{p}=\left[K_{p 1}, K_{p 2}\right]^{T}$, where $K_{p i}$ is from (4.6), $K_{p}$ is an invertible matrix. Choose the control law as

$$
u(t)=K_{P}^{-1} v(t), v(t)=\left[\begin{array}{ll}
v_{1}(t) & v_{2}(t)
\end{array}\right]^{T}
$$

Then (4.48) becomes

$$
y^{(2)}(t)=C_{i} A^{2} x(t)+v_{i}(t)+C_{i} A B_{d} \delta(t)+C_{i} B_{d} \dot{\delta}(t)
$$

Choosing $v_{i}(t)$ as

$$
v_{i}(t)=-C A^{2} x(t)-a_{i 1}^{*} \dot{y}_{i}-a_{i 0}^{*} y(t)+r_{i}(t)-C_{i} A B_{d} \delta(t)-C_{i} B_{d} \dot{\delta}(t)
$$

and applying this $v_{i}(t)$ back to $(4.50)$, we have

$$
\begin{aligned}
& y_{i}^{(2)}(t)+a_{i 1}^{*} \dot{y}_{i}+a_{i 0}^{*} y(t)=r_{i}(t) \\
& y_{i}(s)=\frac{1}{d_{i}(s)} r_{i}(s)
\end{aligned}
$$

that is, we have

$$
y(t)=W_{m}(s) r(t)
$$

From discussion above, with the fact that $\rho_{i}-v_{i}=1$ known, we will need the 
knowledge of $\dot{\delta}(t)$ to design the controller. The signal $v(t)$ can be expressd as

$$
v(t)=K_{0} x(t)+r(t)+K_{d} \delta(t)+K_{d 1} \dot{\delta}(t)
$$

where

$$
\begin{aligned}
& K_{0}=\left[k_{01}^{T}, k_{02}^{T}\right]^{T}, k_{0 i}^{T}=-C_{i} A^{2}-a_{i 1}^{*} C_{i} A-a_{i 0}^{*} C_{i} \\
& K_{d}=\left[k_{d 1}^{T}, k_{d 2}^{T}\right]^{T}, k_{d i}=-C_{i} A B_{d} \\
& K_{d} 1=\left[k_{d 11}^{T}, k_{d 12}^{T}\right]^{T}, k_{d 1 i}=-C_{i} B_{d} .
\end{aligned}
$$

From the definition of $u(t)$ in (4.49), we have

$$
u(t)=K_{p}^{-1}\left(K_{0} x(t)+r(t)+K_{d} \delta(t)+K_{d 1} \dot{\delta}(t)\right)=K_{1}^{*} x(t)+K_{2}^{*} r(t)+K_{3}^{*}(t),
$$

that is,

$$
K_{1}^{*}=K_{p}^{-1} K_{0}, K_{2}^{*}=K_{p}^{-1}, K_{3}^{*}=K_{p}^{-1} K_{d 1}
$$

With the derivation above, the following theorem is obtained.

Theorem 4.2. For the MIMO system under Assumptions 1 and 2, with relative degree conditions $\rho_{i}>v_{i}$, there exist a state feedback control law to achieve disturbance rejection and output tracking: $y(t)=W_{m}(s) r(t)$ if the knowledge of the $\left(\rho_{i}-v_{i}\right)$ th derivative of the disturbance signal $\delta(t)$ is known.

Remark 4.1. When the relative degrees $\rho_{i}<v_{i}$ for all $i=1,2, \ldots, M$, then no disturbance rejection is needed because all the disturbance terms $C A^{\rho_{i}-1} B_{d} \delta(t)$ will becomes zero and this will make $K_{3}^{*}=0$ as $K_{d}=0$. As a result the controller will becomes $u(t)=K_{1}^{*} x(t)+K_{2}^{*} r(t)$. 
Matching Properties for $K_{1}^{*}, K_{2}^{*}, K_{3}^{*}(t)$ From (4.25), we can find the matching properties $K_{1}^{*}, K_{2}^{*}$ and $K_{3}^{*}$ :

$$
\begin{aligned}
& C\left(s I-A-B K_{1}^{* T}\right)^{-1} B K_{2}^{* T}=W_{m}(s) \\
& W_{m}(s) K_{2}^{*-1} K_{3}^{*}(s)+C\left(s I-A-B K_{1}^{* T}\right)^{-1} B_{d} d(s)=0 .
\end{aligned}
$$

There exist constant matrices $K_{1}^{*} \in R^{6 \times 2}$ and $K_{2}^{*} \in R^{2 \times 2}$, and a nonsingular matrix function $K_{3}^{*}(t) \in R^{2} 2 \times 2$ that satisfy the matching equations above.

With parameters satisfying the matching condition above, substituting the matching equations to (4.54), we have that the nominal controller (4.59) ensures that all signals in the closed-loop system are bounded and $\lim _{t \rightarrow \infty}\left(y(t)-y_{m}(t)\right)=0$

Parameterlization of $K_{3}^{*}(t)$ Before the adaptive control design, futher linear parameterization for the term $K_{3}^{*}(t)$ is needed. For the disturbance discribed in (4.2), the elements in $\delta_{j}(t)$ can be characterized as

$$
\delta_{j}(t)=\delta_{j 0}+\sum_{k=1}^{q} \delta_{j k} f_{j k}(t), j=1,2
$$

where $\delta_{j 0}$ and $\delta_{j k}$ are unknown constants and

$$
f_{j}(t)=[\cos (6 \omega t), \sin (6 \omega t), \cos (12 \omega t), \sin (12 \omega t)], j=1,2,
$$

are some known sinucoidal signals. With the property of the derivative of sinucoidal signals, we have that the derivative of the disturbance $\delta(t)$ will have the same form with (4.63), that is

$$
\dot{\delta}_{j}(t)=\delta_{j 0}^{\prime}+\sum_{k=1}^{q} \delta_{j k}^{\prime} f_{j k}(t), j=1,2
$$


where $\delta_{j 0}^{\prime}, \delta_{j k}^{\prime}$ are unknown constants and $f_{j}(t)$ stays the same. Since the disturbance and its derivative have the same form. We have

$$
\begin{aligned}
K_{3}^{*}(t) & =K_{p}^{-1}\left(K_{d} \delta(t)+K_{d 1} \dot{\delta}(t)\right) \\
& =K_{p}^{-1}\left(K_{d} \Phi^{*} f(t)+K_{d 1} \Phi^{\prime *} f(t)\right)=K_{3 f}^{*} f(t),
\end{aligned}
$$

where

$$
\begin{aligned}
\delta(t) & =\Phi^{* T} f(t), \\
f(t) & =[1, \cos (6 \omega t), \sin (6 \omega t), \cos (12 \omega t), \sin (12 \omega t), \\
1, \cos (6 \omega t), \sin (6 \omega t), \cos (12 \omega t), \sin (12 \omega t)]^{T} \in R^{10}, & \\
\Phi^{*} & =\left[\begin{array}{ll}
\phi_{1}^{* T} & 0_{(5)}^{T} \\
0_{(5)}^{T} & \phi_{2}^{T *}
\end{array}\right] \in R^{2 \times 10}, \\
\phi_{i} & =\left[\begin{array}{ll}
\delta_{i 0}, \ldots, \delta_{i 5}
\end{array}\right] \in R^{5} \\
\dot{\delta}(t) & =\tilde{\Phi}^{* T} f(t), \\
\Phi^{* *} & =\left[\begin{array}{ll}
\phi_{1}^{\prime * T} & 0_{(5)}^{T} \\
0_{(5)}^{T} & \phi_{2}^{T *}
\end{array}\right] \in R^{2 \times 10}, \\
\phi_{i}^{\prime} & =\left[\begin{array}{ll}
\delta_{i 0}^{\prime}, \ldots, \delta_{i 5}^{\prime}
\end{array}\right] \in R^{5} \\
K_{3 f}^{*} & =K_{p}^{-1}\left(K_{d} \Phi^{*}+K_{d 1} \Phi^{\prime *}\right) .
\end{aligned}
$$

With the derivation above, we can propose the following proposition

Proposition 4.3. The disturbance signal $\delta(t)$ in (4.63) being the summation of sinusoidal signals, the disturbance related control parameter $K_{3}^{*}(t)=K_{p}^{-1}\left(K_{d} \delta(t)+\right.$ $\left.K_{d 1} \dot{\delta}(t)\right)$ can be parameterized to the form $K_{3 f}^{*} f(t)$, where $f(t)$ contains all the fundamental sinusoidal signals. 
Next, the adaptive version of the control scheme is studied for systems witout the knowledge of system and disturbance parameters.

\subsubsection{Adaptive Disturbance Rejection Design}

Based the nominal controller with disturbance rejection obtained from the previous section, in this subsection a state feedback output tracking MRAC scheme with disturbance rejection, for the three-phase PV inverter working in the grid-connected mode, is proposed.

Controller Structure The adaptive controller has the same structure as the nonminal controller (4.13):

$$
u(t)=K_{1}^{T}(t) x(t)+K_{2}(t) r(t)+K_{3}(t),
$$

where $K_{1} \in R^{6 \times 2}, K_{2} \in R^{2 \times 2}$ and $K_{3}(t) \in R^{2}$ are estimates of $K_{1}^{*}, K_{2}^{*}$ and $K_{3}^{*}(t)$. In view of (4.46), we use

$$
K_{3}(t)=K_{3 f}(t) f(t)
$$

where $K_{3 f}(t)$ is the estimate of $K_{3 f}^{*}$ in $(4.76)$.

Tracking Error Equation Apply the adaptive controller to the plant (4.1), we can obtain the following closed-loop system

$$
\begin{aligned}
\dot{x}(t) & =\left(A+B K_{1}^{* T}\right) x(t)+B K_{2}^{*} r(t)+B K_{3}^{*}(t)+B_{d} \delta(t)+B\left(K_{1}^{T}-K_{1}^{* T}\right) x(t) \\
& +B\left(K_{2}-K_{2}^{*}\right) r(t)+B\left[K_{3}(t)-K_{3}^{*}(t)\right] \\
y(t) & =C x(t) .
\end{aligned}
$$


From the definition of the reference signal (4.4), the parameter matching properties (4.61)-(4.62), and this obtained closed-loop system, we can obtain the output tracking error equation as

$$
\begin{aligned}
e(t) & =y(t)-y_{m}(t) \\
& =W_{m}(s) K_{2}^{*-1}\left[\tilde{\Theta}^{T} \omega\right](t)+C e^{\left(A+B K_{1}^{* T}\right) t} x(t),
\end{aligned}
$$

where

$$
\begin{aligned}
\Theta^{* T} & =\left[K_{1}^{* T}, K_{2}^{*}, K_{3 f}^{*}\right] \in R^{2 \times 13} \\
\omega(t) & =\left[x^{T}(t), r^{T}(t), f^{T}(t)\right]^{T} \in R^{13} \\
\Theta^{T}(t) & =\left[K_{1}^{T}(t), K_{2}(t), K_{3 f}^{T}\right] \in R^{2 \times 13} \\
\tilde{\Theta}(t) & =\Theta(t)-\Theta^{*}
\end{aligned}
$$

The term $C e^{\left(A+B K_{1}^{* T}\right) t} x(t)$ converge to zero exponentially fast due to the stability of $A+B K_{1}^{* T}$. As a result we can just write the output tracking error as

$$
e(t)=y(t)-y_{m}(t)=W_{m}(s) K_{2}^{*-1}\left[\tilde{\Theta}^{T} \omega\right](t)
$$

Estimation Error In order to develop the adaptive laws to update the controller parameter $\Theta(t)$, we need further define the estimation error. First choose the filter $h(s)=\frac{1}{f(s)}$, where $f(s)$ is a stable polynomial $f(s)=s^{2}+f_{1} s+f_{0}$ of degree 2 . Then we can define the estimation error as

$$
\varepsilon(t)=\xi_{m}(s) \Lambda(s)\left[y-y_{m}\right](t)+K_{p}(t) \xi(t)=\xi_{m}(s) \Lambda(s)[e](t)+K_{p}(t) \xi(t) .
$$


There $K_{p}(t)$ is the estimate of the high-frequency gain matrix $K_{p}^{*}$ in Assumption 2, $\xi_{m}(s)$ is the diagonal interactor matrix $\xi_{m}(s)=\operatorname{diag}\left\{d_{1}(s), d_{2}(s)\right\}$, and

$$
\begin{aligned}
& \xi(t)=\Theta^{T}(t) \zeta(t)-h(s)[u](t) \\
& \zeta(t)=h(s)[\omega](t) .
\end{aligned}
$$

With the output tracking error equation (4.82), we can rewrite the estimation error as

$$
\varepsilon(t)=K_{p}^{*} \tilde{\Theta}(t) \zeta(t)+\tilde{K}_{p}(t) \xi(t), \tilde{K}_{p}(t)=K_{p}(t)-K_{p}^{*}
$$

Adaptive Laws Based on the estimation error equation (4.92), the adaptive parameter update laws are chosen as

$$
\begin{aligned}
& \dot{\Theta}^{T}(t)=-\frac{\Gamma_{1} \varepsilon(t) \zeta^{T}(t)}{m^{2}(t)} \\
& \dot{K}_{p}(t)=-\frac{\Gamma_{2} \varepsilon(t) \xi^{T}(t)}{m^{2}(t)}
\end{aligned}
$$

where $\Gamma_{i}=\Gamma_{i}^{T}>0 i=1,2$, and $m^{2}(t)=1+\zeta^{T}(t) \zeta(t)+\xi^{T}(t) \xi(t)$.

Stability Analysis With the adaptive law design above, we can prove the following lemma is true for our adaptive laws.

Lemma 4.3. The adaptive laws (4.93), and (4.94) guarantee that

(i) $\Theta(t) \in L^{\infty}, K_{p}(t) \in L^{\infty}, \frac{\varepsilon(t)}{m(t)} \in L^{2} \cap L^{\infty}$;

(ii) $\dot{\Theta}(t) \in L^{\infty} \cap L^{2}, \dot{K}_{p}(t) \in L^{\infty} \cap L^{2}$.

Proof. Choose the positive definite function

$$
V=\frac{1}{2}\left(\operatorname{tr}\left[\tilde{K}_{p}^{T} \Gamma_{2}^{-1} \tilde{K}_{p}\right]+\operatorname{tr}\left[\tilde{\Theta}^{T} \Gamma_{1}^{-1} \tilde{\Theta}\right]\right)
$$


With the adaptive laws (4.93), (4.94), the time derivetive of $\mathrm{V}$ can be derived as

$$
\dot{V}=-\frac{\varepsilon^{T}(t) \varepsilon(t)}{m^{2}(t)} \leq 0 .
$$

Thus, we can conclude that $\Theta(t) \in L^{\infty}, K_{p}(t) \in L^{\infty}, \frac{\varepsilon(t)}{m(t)} \in L^{2} \cap L^{\infty}, \dot{\Theta}(t) \in L^{\infty} \cap L^{2}$, $\dot{K}_{p}(t) \in L^{\infty} \cap L^{2}$

Based on Lemma 4.3, one can ensure that the closed-loop system has the desired properties.

Theorem 4.3. For the plant (4.1) with uncertain system parameters and under Assumptions 1 and 2 and the reference model (4.4), the adaptive controller (4.74) with the adaptive update laws (4.93), and (4.94) guarantee all signals in the closedloop system are bounded and the output tracking error $e(t)=y(t)-y_{m}(t)$ satisfies $\lim _{t \rightarrow \infty} e(t)=0$.

With this theorem, the performance of the adaptive controller designed in this section can achieve output tracking of the given signal $y_{m}$. The physical meaning of this statement is, the output current can track a reference current signal asymptotically.

\subsection{Simulation Results}

In this section, the simulation results are presented for state feedback output tracking control schemes for inverters working without grid influence and with gridconnection respectively. 


\subsubsection{Inverter System Operating without Disturbance}

For the inverter system working without disturbance, the system state space equation is

$$
\dot{x}(t)=A x(t)+B u(t), y(t)=C x(t),
$$

where

$$
\begin{aligned}
& x(t)=\left[\begin{array}{llllll}
I_{i d} & I_{i q} & I_{g d} & I_{g q} & U_{P V} & U_{q c}
\end{array}\right]^{T}, u(t)=\left[\begin{array}{ll}
d_{d} & d_{q}
\end{array}\right], \\
& A=\left[\begin{array}{cccccc}
-88.89 & 314.16 & 33.33 & 0 & -1111.11 & 0 \\
-314.16 & -88.89 & 0 & 33.33 & 0 & -1111.11 \\
111.11 & 0 & -55.56 & 314.16 & 1851.85 & 0 \\
0 & 111.11 & -314.16 & -55.56 & 0 & 1851.85 \\
33333.33 & 0 & -33333.33 & 0 & 0 & 314.16 \\
0 & 33333.33 & 0 & -33333.33 & -314.16 & 0
\end{array}\right] \text {, } \\
& B=\left[\begin{array}{cccccc}
5.13 \times 10^{5} & 0 & 0 & 0 & 0 & 0 \\
0 & 5.13 \times 10^{5} & 0 & 0 & 0 & 0
\end{array}\right], \\
& C=\left[\begin{array}{llllll}
0 & 0 & 1 & 0 & 0 & 0 \\
0 & 0 & 0 & 1 & 0 & 0
\end{array}\right] \text {. }
\end{aligned}
$$

These nominal values are obtained by applying the system parameters in Table 4.1 to the system matrix $(A, B, C)$ in (3.11)-(3.13). This linear model can be treated as a test bed to do simulations systematically to verify the effectiveness of our proposed adaptive control scheme. 
Table 4.1: Simulation study parameter [17], [30]

\begin{tabular}{|l|l|l|}
\hline Parameter & Unit & Value \\
\hline Inductor $L_{f}$ & $(\mathrm{mH})$ & 0.9 \\
\hline Capacitor $C$ & $(\mu \mathrm{F})$ & 30 \\
\hline Inductor $L_{g}$ & $(\mathrm{mH})$ & 0.54 \\
\hline Resistance $R_{f}$ & $(\mathrm{~m} \Omega)$ & 0.05 \\
\hline Resistance $R_{g}$ & $(\mathrm{~m} \Omega)$ & 0.03 \\
\hline Resistance $R_{c}$ & $(\mathrm{~m} \Omega)$ & 0.05 \\
\hline Line-to-line grid voltage $U_{a, b, c}$ & $(\mathrm{~V})$ & 380 \\
\hline Grid frequency $\omega$ & $(\mathrm{rad} / \mathrm{s})$ & 314.15 \\
\hline Input voltage $U_{P V}$ & $(\mathrm{~V})$ & $750-820$ \\
\hline
\end{tabular}

Adaptive Control Design For the inverter system (4.104), it can be verified that the transfer matrix $G(s)=C(s I-A)^{-1} B$ has stable zeros: $z_{1}=(-5.56-j 0.0031) \times$ $10^{5}, z_{2}=(-5.56+j 0.0031) \times 10^{5}$. Also, this transfer matrix is strictly proper and full rank. Choosing the interactor matrix as $\xi_{m}(s)=\operatorname{diag}\left\{(s+1)^{2},(s+1)^{2}\right\}$, such that the high-frequency gain matrix is

$$
K_{p}=\lim _{s \rightarrow \infty} \xi_{m}(s) G(s)=\left[\begin{array}{cc}
5.7022 \times 10^{7} & 0 \\
0 & 5.7022 \times 10^{7}
\end{array}\right]
$$

which is positive finite and nonsingular. Then the reference model is chosen as

$$
W_{m}(s)=\xi_{m}^{-1}(s)=\operatorname{diag}\left\{\frac{1}{(s+1)^{2}}, \frac{1}{(s+1)^{2}}\right\}
$$

We also choose $f(s)=(s+1)^{2}$ and $h(s)=\frac{1}{(s+1)^{2}}$ for MRAC design.

Simulation Results Choose the initial control parameters 0.8 times of their true nominal values. Choose $\Gamma_{1}=\Gamma_{2}=0.1 I$. There are two cases of simulation results. In Case 1 , the system tracks a constant output $y_{m}=\left[I_{g d}^{*}, I_{g q}^{*}\right]^{T}$, where $I_{g d}^{*}=\frac{2 P^{*}}{3 U_{d} N}=$ $17 A, I_{g q}=0$. In Case 2 , the system tracks $y_{m}(t)$ which initially has the same value 
as in Case 1 and then increases as time goes.

Case 1: Choose $r(t)=[17,0]^{T}, y_{m}(0)=[17,0]^{T}$. In this way, $y_{m}$ is just constant signal. Figure $4.1 \mathrm{~b}$ shows the output current and the tracking error in the d-q axis. We can see that the system achieved desired tracking after 0.1 seconds. Figure $4.1 \mathrm{a}$ shows the three phase output currents, from which we can see that the system output current tracks $y_{m}(t)$ asymptotically.

Case 2: Choose $r(t)=[17,0]^{T} t \leq 1, r(t)=[20,0]^{T}, t>1$, this way, $y_{m}$ changes smoothly. Figure 4.2a shows the desired tracking signal $y_{m}(t)$ which is a slowly increasing signal. Figure. 4.2b show the output current and the tracking error in $\mathrm{d}-\mathrm{q}$ axis. This result shows that if $y_{m}(t)$ is a time variant signal, the adaptive controller designed for this situation can still achieve tracking. This is something that a classic controller cannot do.

The inverter system works with no grid influence is a simulation for the case where the inverter system have not been connected to the grid yet. This is a good start and practice design before considering the case where the inverter is actually connected in grid. The simulation results for these cases shows the good performance of the adaptive controller for the electrical circuit systems. 


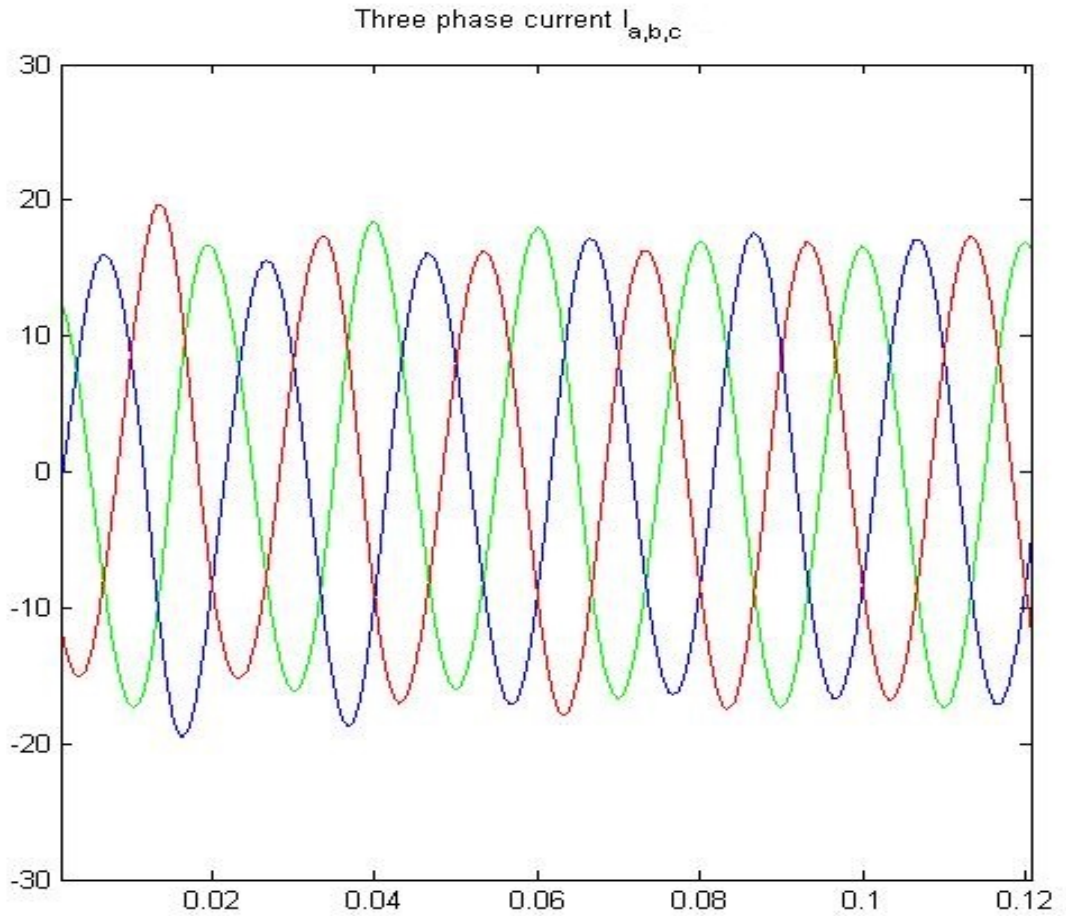

(a) The output current in the a-b-c reference frame.
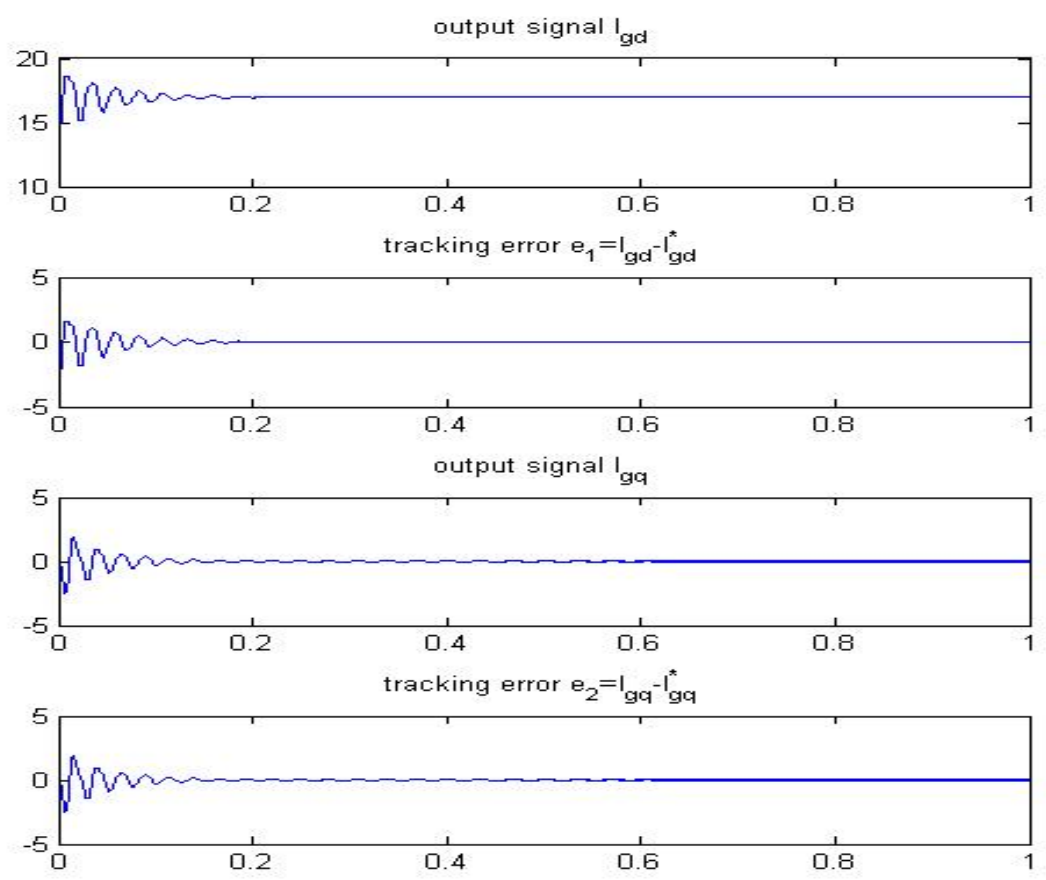

(b) The output current and tracking error of $I_{g d}, I_{g q}$.

Figure 4.1: System response for Case 1. 

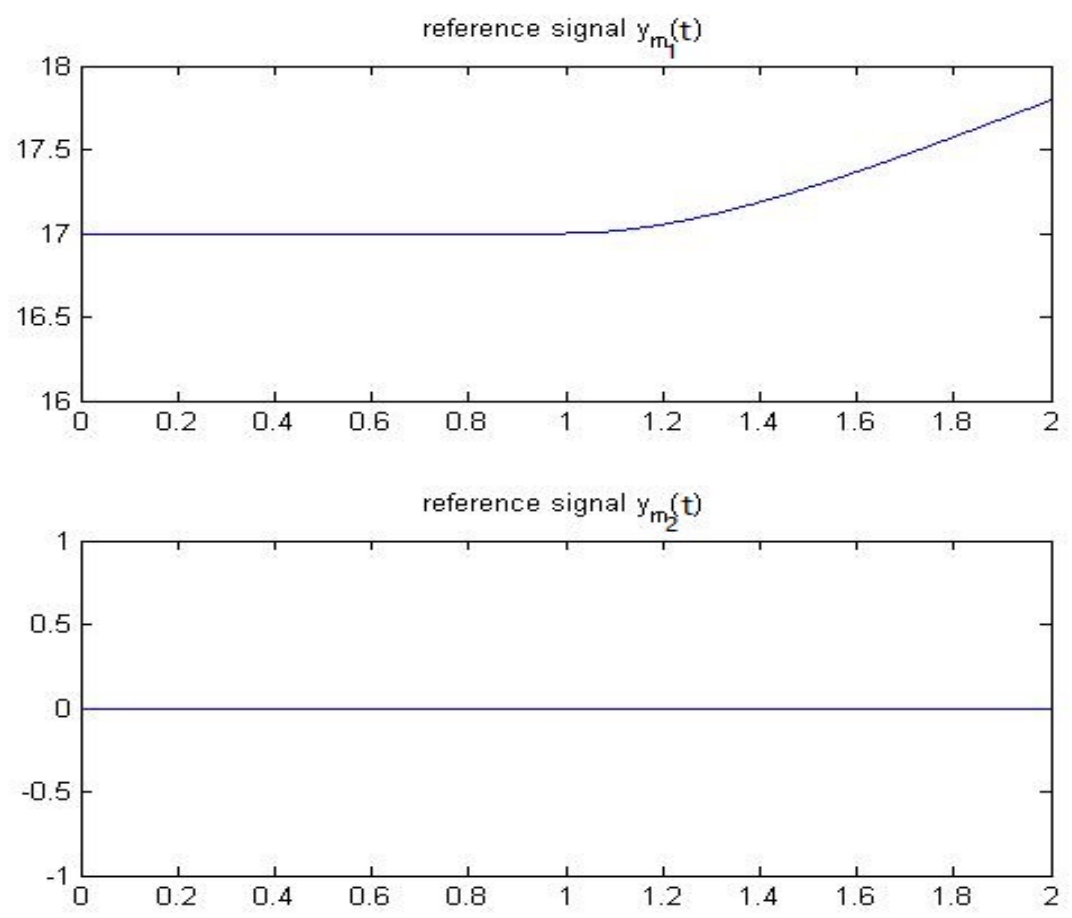

(a) The reference signal $y_{m}(t)$.
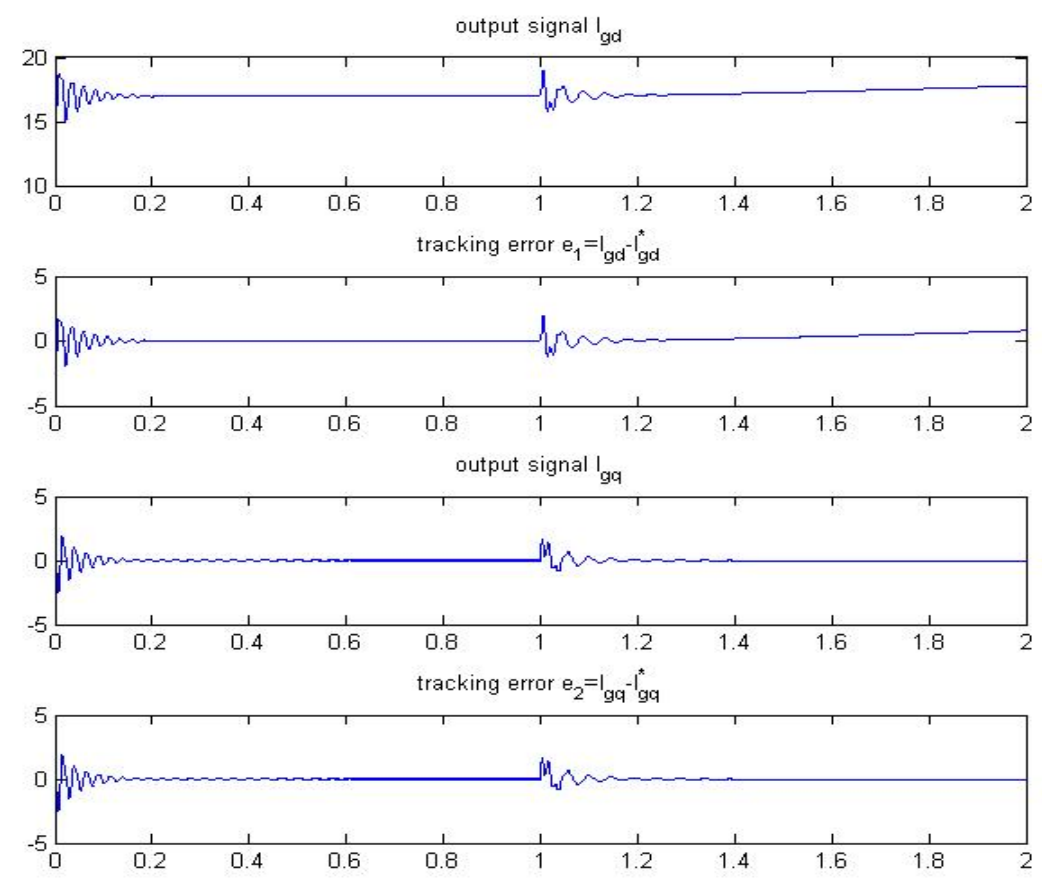

(b) The output current and tracking error of $I_{g d}, I_{g q}$.

Figure 4.2: System response for Case 2. 


\subsubsection{Inverter System Grid-Connected}

For the inverter system working grid-connected, the system state space equation is

$$
\dot{x}(t)=A x(t)+B u(t)+B_{d} \delta(t), y(t)=C x(t),
$$

where $A, B, C$ and the definitions of $x(t)$ and $u(t)$ are stated in Section 4.5.1. and

$$
\begin{aligned}
B_{d} & =\left[\begin{array}{cccccc}
0 & 0 & -1.28 \times 10^{4} & 0 & 0 & 0 \\
0 & 0 & 0 & -1.28 \times 10^{4} & 0 & 0
\end{array}\right]^{T}, \\
\delta(t) & =\left[\begin{array}{l}
U_{d N}(t) \\
U_{q N}(t)
\end{array}\right] .
\end{aligned}
$$

Adaptive Control Design For the inverter system (4.104), it can be verified that the transfer matrix $G(s)=C(s I-A)^{-1} B$ has stable zeros: $z_{1}=(-5.56-j 0.0031) \times$ $10^{5}, z_{2}=(-5.56+j 0.0031) \times 10^{5}$. Also, this transfer matrix is strictly proper and full rank. Choosing the interactor matrix as $\xi_{m}(s)=\operatorname{diag}\left\{(s+1)^{2},(s+1)^{2}\right\}$, such that the high-frequency gain matrix is

$$
K_{p}=\lim _{s \rightarrow \infty} \xi_{m}(s) G(s)=\left[\begin{array}{cc}
5.7022 \times 10^{7} & 0 \\
0 & 5.7022 \times 10^{7}
\end{array}\right],
$$

being positive finite and nonsingular and the matrix

$$
K_{d}=\lim _{s \rightarrow \infty} \xi_{m}(s) G_{d}(s)=\left[\begin{array}{cc}
-1.8519 \times 10^{3} & 0 \\
0 & -1.8519 \times 10^{3}
\end{array}\right],
$$


is finite. Then the reference model is chosen as

$$
W_{m}(s)=\xi_{m}^{-1}(s)=\operatorname{diag}\left\{\frac{1}{(s+1)^{2}}, \frac{1}{(s+1)^{2}}\right\}
$$

We also choose $f(s)=(s+1)^{2}$ and $h(s)=\frac{1}{(s+1)^{2}}$ for MRAC design.

Simulation Results Choose $\Gamma_{1}=\Gamma_{2}=0.1 I$. There are three cases of simulation results presented. Case 3 shows the inverter system working in a standard grid which means $\delta(t)=[310,0]^{T}$, for this case the ability of the adaptive controller to work normally in the grid is tested (with initial parameters equal to the true nominal parameters). Case 4 shows the inverter working in the standard grid with system parameters unknown (choosing the initial control parameters 0.8 times of their true nominal values), this will test the ability to deal with system uncertainties for the adaptive controller. Case 5 shows the inverter working in a polluted grid, i.e., the grid side voltage have some harmonic components. This case will show the disturbance rejection ability of the controller to work under unknown harmonic disturbance.

Case 3: Choose $r(t)=[17,0]^{T}, y_{m}(0)=[17,0]^{T}, \delta(t)=[310,0]^{T}$. Figure 4.3b shows the output current and the tracking error in the $d-q$ axis, from which we can see that for the inverter system works normally in the grid, the output grid side current can track the desired output current value. Figure 4.3a shows the three-phase output currents, from which we can see that the system achieved the desired output current. Figure 4.4 shows the fast Fourier transformation (FFT) analysis of the three-phase output current, where the sampling interval for the FFT analysis is $5 \times 10^{-5}$. We can see that by using our control method, the THD is $0.01 \%$ in this case. 


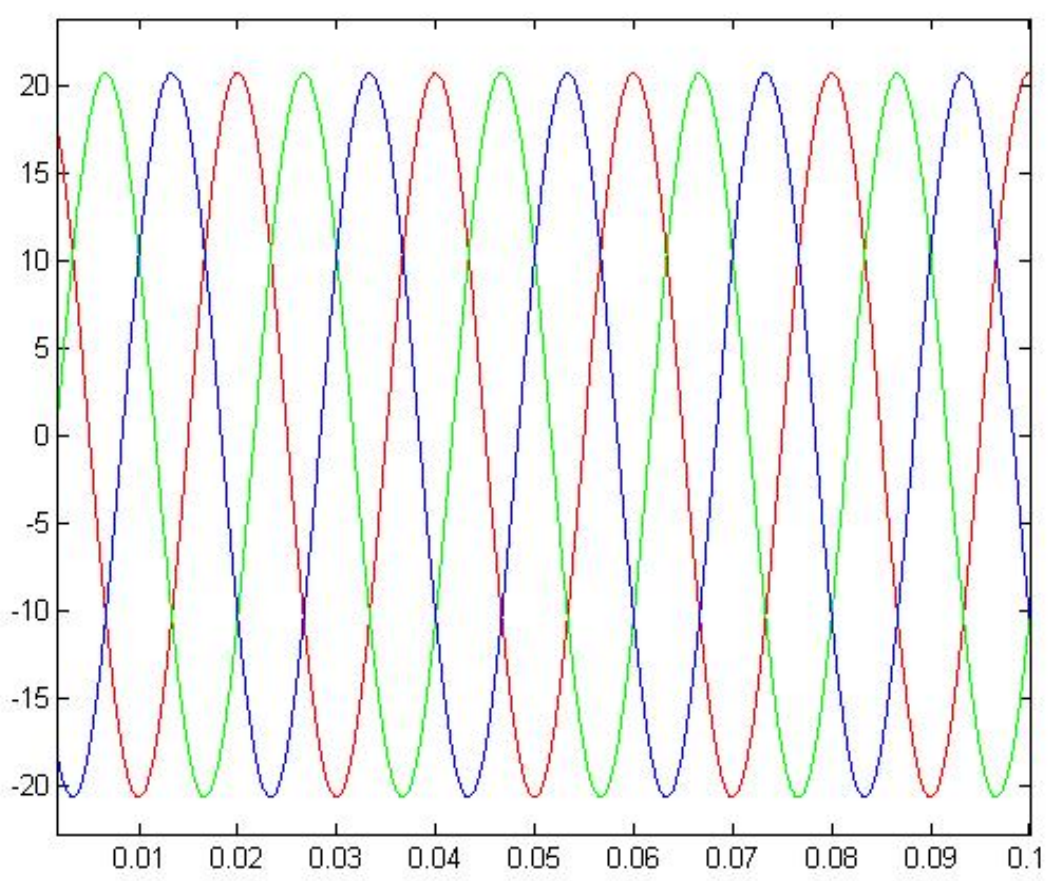

(a) The output current in the a-b-c reference frame.
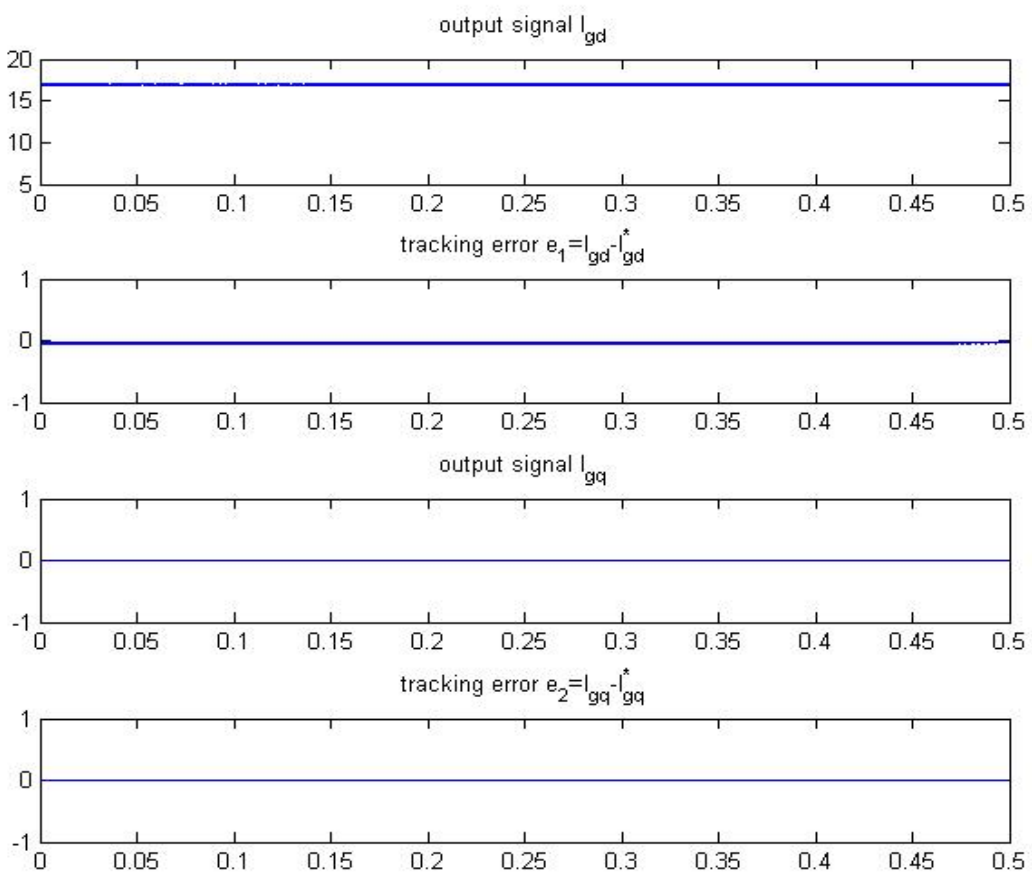

(b) The output current and tracking error of $I_{g d}, I_{g q}$.

Figure 4.3: System response for Case 3. 

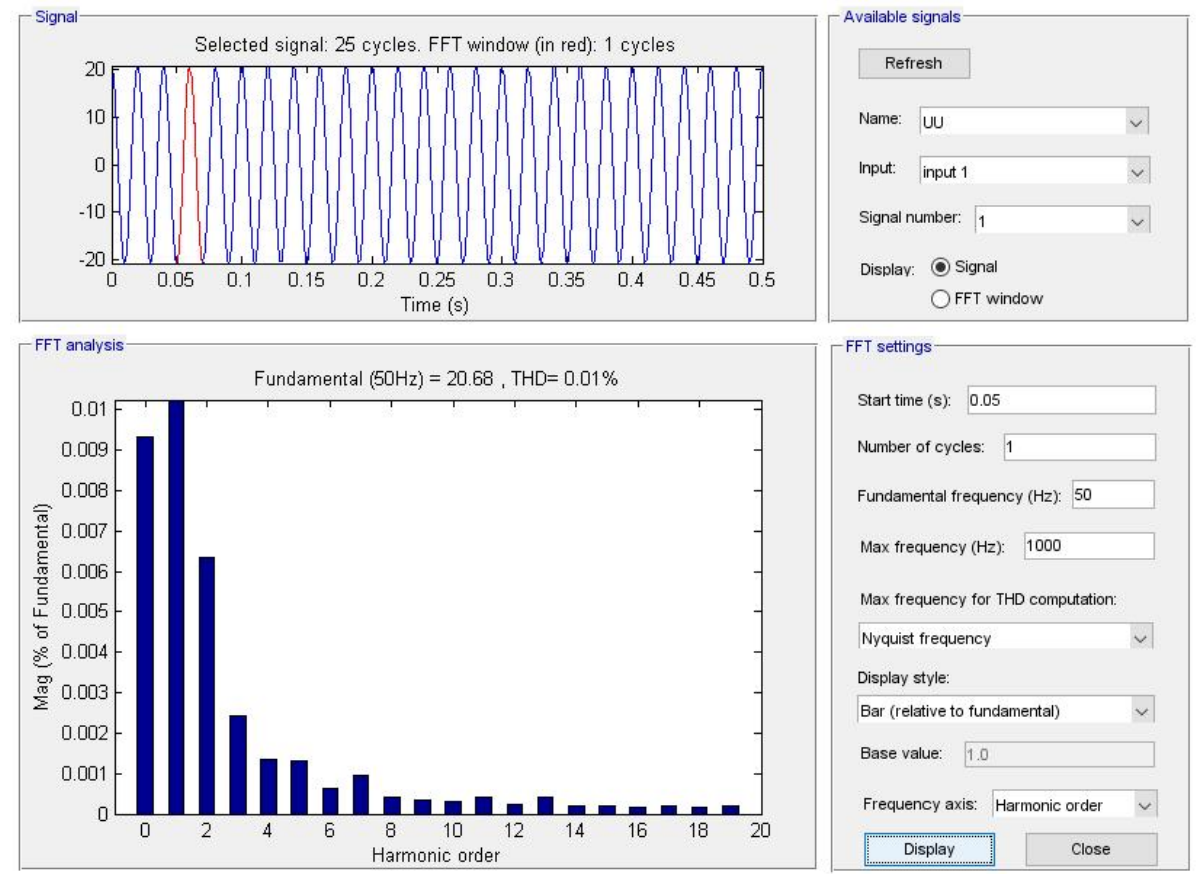

Figure 4.4: FFT analysis for the output current $I_{g a}$ for Case 3.

Case 4: Choose $r(t)=[17,0]^{T}, y_{m}(0)=[17,0]^{T}, \delta(t)=[310,0]^{T}$, all initial control parameters are 0.8 times of their true nominal values. Figure $4.5 \mathrm{~b}$ shows the output current and the tracking error in the $\mathrm{d}-\mathrm{q}$ axis, from which we can see that the system achieved desired tracking and disturbance rejection before 0.1 seconds. Figure 4.5a shows the three phase output current, from which we can see that the system achieved the desired output current. Figure 4.6 shows the FFT analysis of the output current, with the sampling interval $5 \times 10^{-5} \mathrm{~s}$. We can see that by using our control method, the THD in this case is $0.26 \%$. 


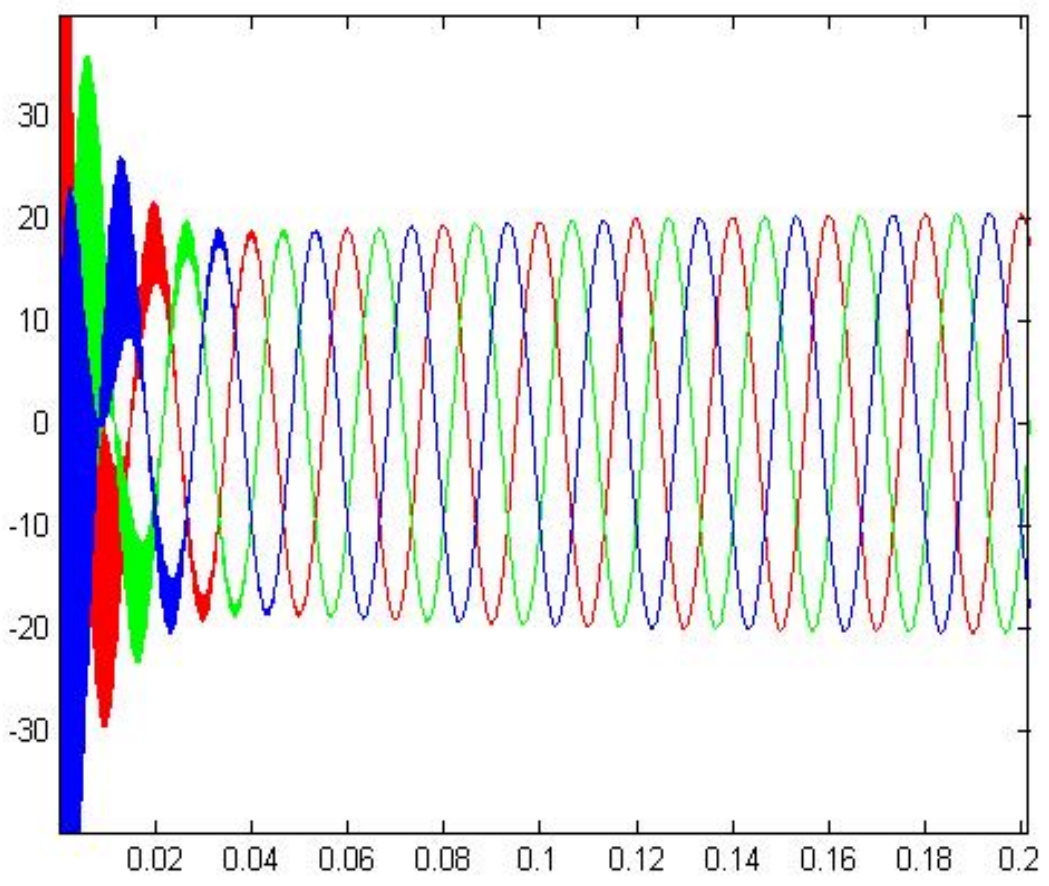

(a) The output current in the a-b-c reference frame.
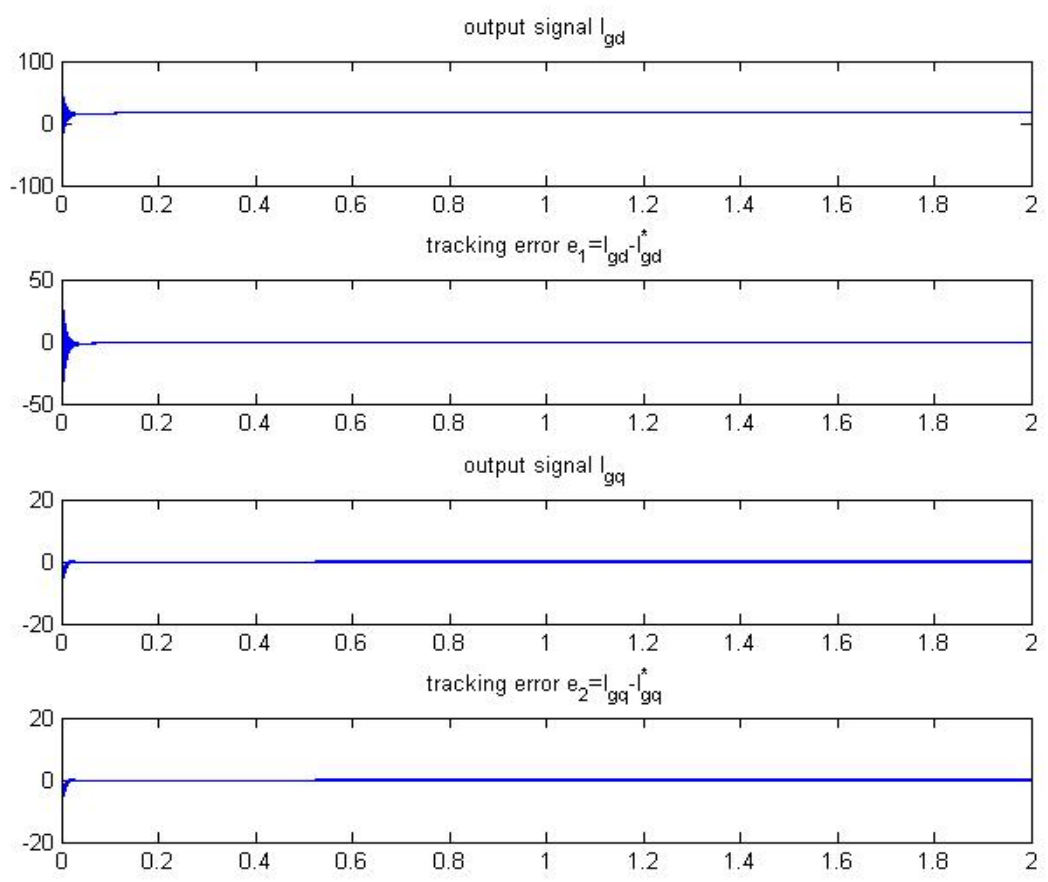

(b) The output current and tracking error of $I_{g d}, I_{g q}$.

Figure 4.5: System response for Case 4. 

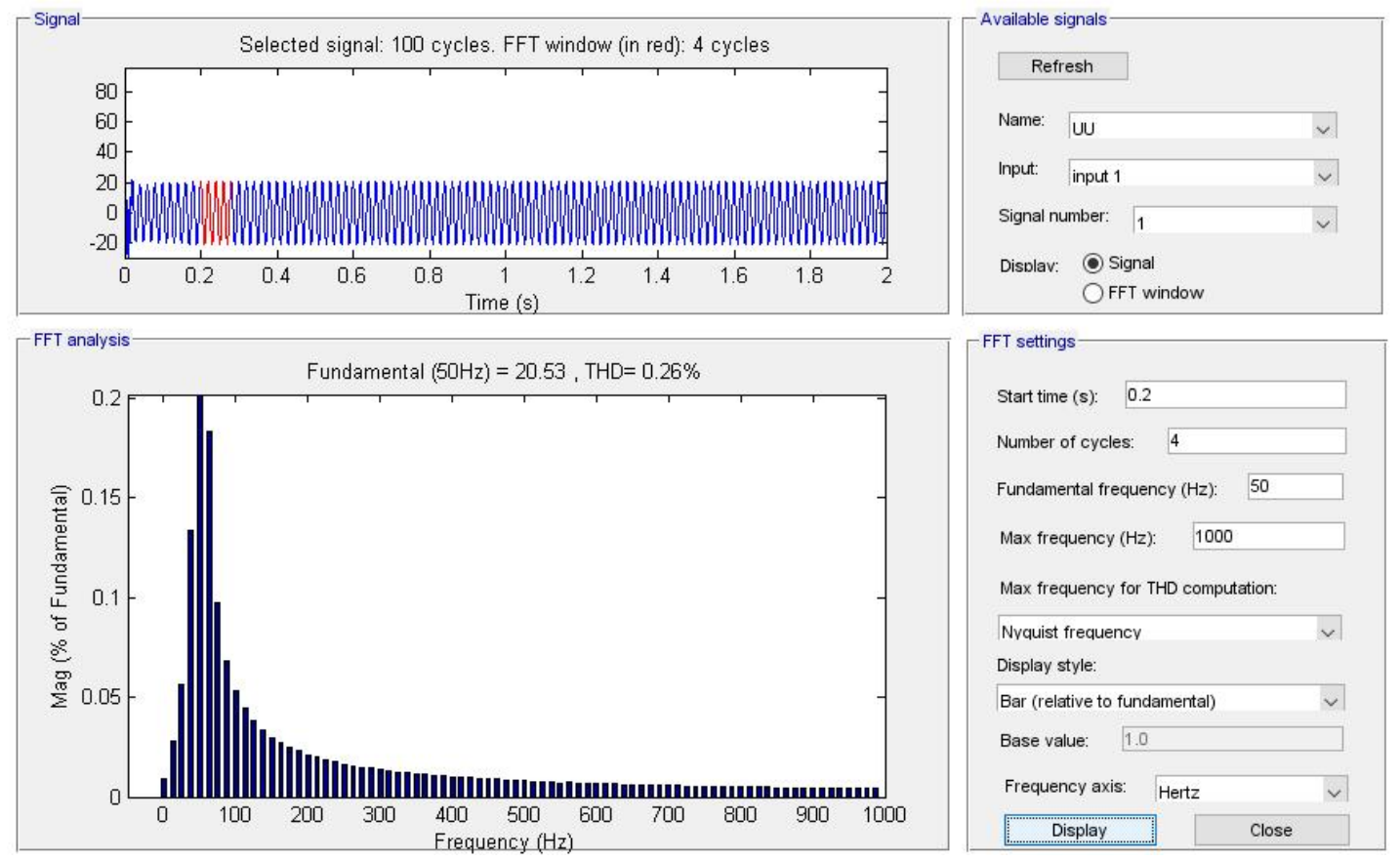

Figure 4.6: FFT analysis for the output current $I_{g a}$ for Case 3.

Case 5: Choose $r(t)=[17,0]^{T}, y_{m}(0)=[17,0]^{T}$. The polluted grid is considered as the disturbance

$$
\delta(t)=\left[\begin{array}{c}
310+10 \cos (6 \omega t)+10 \sin (6 \omega t)+5 \cos (12 \omega t)+5 \sin (12 \omega t) \\
10 \cos (6 \omega t)+10 \sin (6 \omega t)+5 \cos (12 \omega t)+5 \sin (12 \omega t)
\end{array}\right] .
$$

The THD of the grid side voltage is $6.2 \%$. Figure $4.7 \mathrm{~b}$ shows the output current and the tracking error in the d-q axis. Figure 4.7a shows the three phase output current. We can see that the system achieved the desired output current. Figure 4.8 shows the FFT analysis of the output current, with the sampling interval $5 \times 10^{-5}$ s. We can see that by using our control method, the THD, in this case, is $3.9 \%$, less than the grid side THD. 


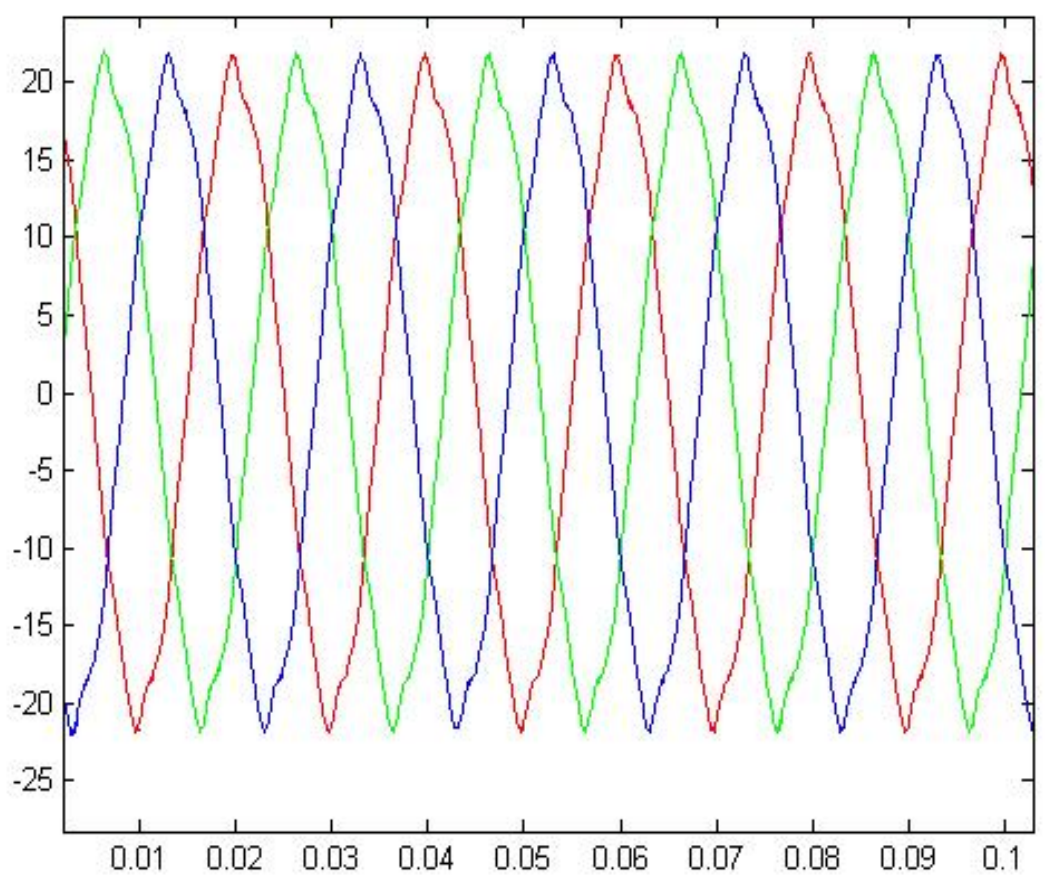

(a) The output current in the a-b-c reference frame.
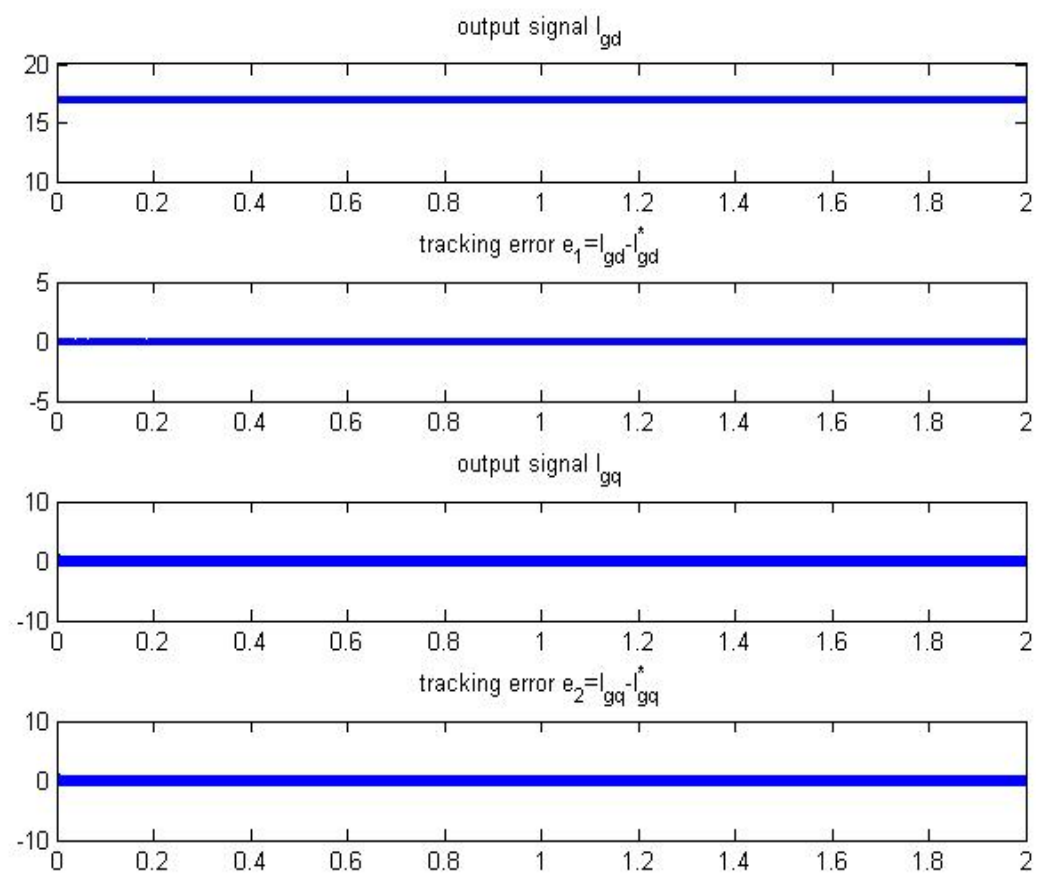

(b) The output current and tracking error of $I_{g d}, I_{g q}$.

Figure 4.7: System response for Case 5. 

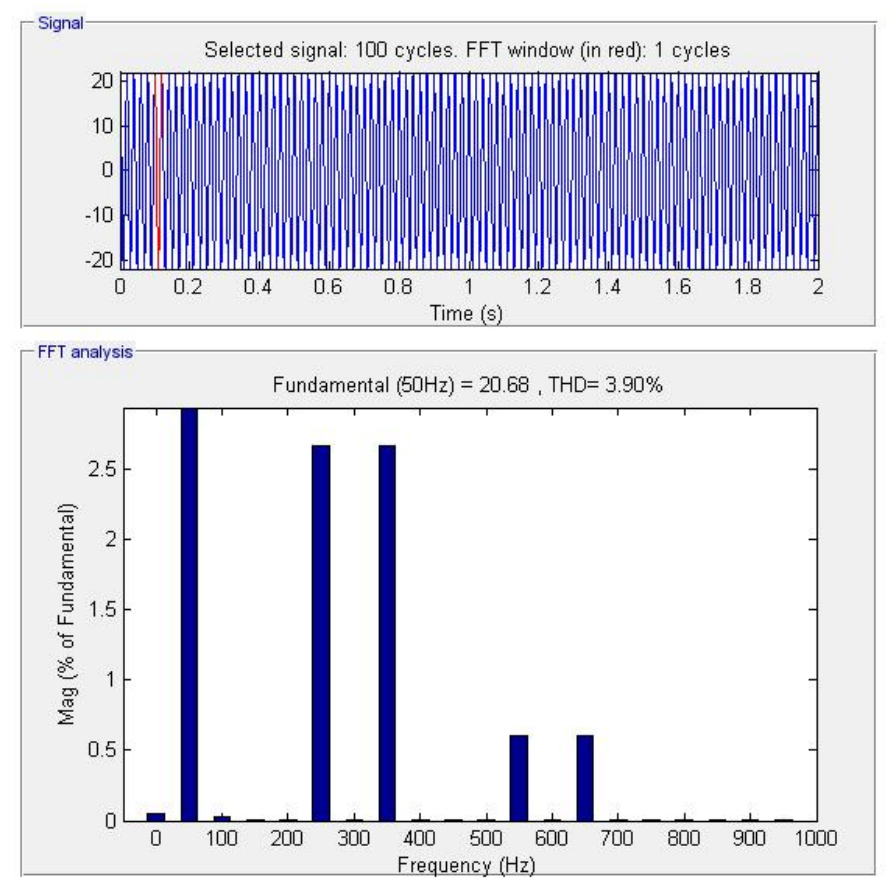
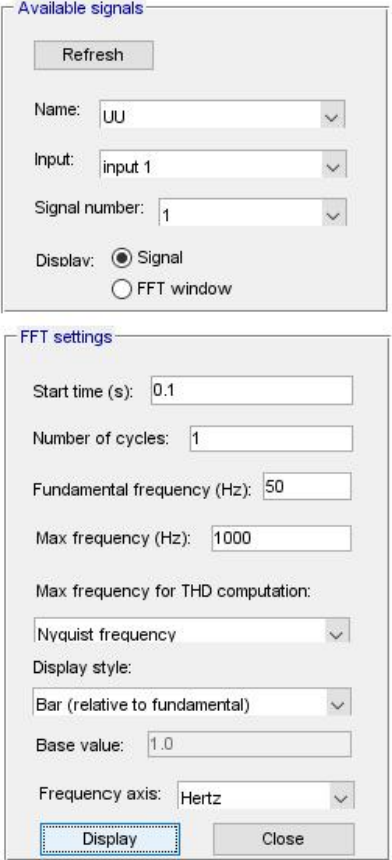

Figure 4.8: FFT analysis for the output current $I_{g a}$ for Case 5.

\subsection{Summary}

In this chapter, the state feedback adaptive control schemes for the three-phase inverter system working without grid influence and with grid-connected are designed respectively. Simulation results are presented to verify our designs. From the simulation result, we can conclude that the control objective is achieved. Firstly, our controller can normally work in a grid when there is no parameter uncertainty. Secondly, our controller can deal with the case where the system parameters and the disturbance parameters are unknown, and our controller can handle the tracking for the case where the reference signal is changing. As a comparison, a classical PI controller cannot handle a varying reference signal. Being able to track a time variant signal is useful in the control problem in the photovoltaic inverter systems due to the uncertainties of the system parameters for the photovoltaic power generation devices. 


\section{Chapter 5}

\section{Output Feedback Adaptive Control \\ Scheme}

In this chapter, a output feedback output tracking MRAC scheme is derived to control the main circuit of the three-phase PV inverter. Similarly, the control scheme for the case where the PV inverter working without the grid influence, and then designed for the general case where the PV inverter works with the grid-connection. Simulation results are shown at the end of the chapter to verify the control designs.

\subsection{Problem Formulation}

The system model has been derived in Chapter 3 and its restated here. Consider the linear model for the three-phase PV inverter system:

$$
\dot{x}(t)=A x(t)+B u(t)+B_{d} \delta(t), y(t)=C x(t),
$$

where $A \in R^{6 \times 6}, B \in R^{6 \times 2}, B_{d} \in R^{6 \times 2}$, and $C \in R^{2 \times 6}$ are unknown constant

matrices, with $x(t) \in R^{6}, y(t) \in R^{2}$ being available for measurement. They are 
obtained from Section 3.3. In this model, $\delta(t) \in R^{2}$ is an unmatched disturbance of the system because $B$ and $B_{d}$ are linearly independent, which can be written in the form

$$
\begin{aligned}
\delta(t) & =\left[\begin{array}{c}
\delta_{10}+\sum_{k=1}^{6} \delta_{1 k} f_{1 k}(t) \\
\delta_{20}+\sum_{k=1}^{6} \delta_{2 k} f_{2 k}(t)
\end{array}\right] \\
& =\left[\begin{array}{c}
U_{d N}(t) \\
U_{q N}(t)
\end{array}\right]=\left[\begin{array}{c}
U_{d 1 N}+U_{d 6 c N} \cos (6 \omega t)+U_{d 6 s N} \sin (6 \omega t)+\ldots \\
U_{q 6 c N} \cos (6 \omega t)+U_{q 6 s N} \sin (6 \omega t)+\ldots
\end{array}\right] .
\end{aligned}
$$

Notice that $B_{d} \neq \alpha B$ for any $\alpha \in R^{2 * 2}$, for the obtained form of $B$ and $B_{d}$ of the three-phase grid-connected PV inverter system.

The control objective for this adaptive control problem with disturbance compensation is to design an adaptive controller to generate $u(t)$ to guarantee the closed-loop signal boundedness and to make the output signal $y(t)$ to track a chosen reference signal generated from a reference model:

$$
y_{m}(t)=W_{m}(s)[r](t)=\left[\begin{array}{c}
I_{g d}^{*} \\
I_{g q}^{*}
\end{array}\right] \in R^{2}, \quad t \geq 0
$$

where $W_{m}(s) \in R^{2 \times 2}$ is a stable transfer function matrix, and $r(t) \in R^{2}$ is the reference input signal which can be defined when $y_{m}(t)$ is chosen. For the control problem of three phase PV inverter system, we can always obtain the desired reference signal $I_{g d}^{*}$ and $I_{g q}^{*}$, where $I_{g q}^{*}$ is always set to be zero. From the discussion in Section 3.4 when the grid side is under three phase balance condition $I_{g d}^{*}=\frac{2 P^{*}(t)}{3 U_{d 1 N}}$, where $P^{*}$ can be obtained from the MPPT module. Note that when the grid side is operating under three phase unbalance situation, the state-of-art research can still obtain the desired value of $I_{g d}^{*}$ and $I_{g q}^{*}$ for this control problem. 


\subsection{Basic Design Conditions}

The two design conditions in Section 4.2 for state feedback design is still needed in output feedback design, which is restated here. They have been verified in Section 4.2 .

Assumption 1 All zeros of $G(s)=C(s I-A)^{-1} B$ are stable, and (A,B,C) is stabilizable and detectable.

Assumption $2 G(s)$ is strictly proper with full rank and has a diagonal interactor matrix $\xi_{m}=\operatorname{diag}\left\{d_{1}(s), d_{2}(s)\right\}$, where $d_{i}(s)=s^{\rho_{i}}+a_{i \rho_{i}-1}^{*} s^{\rho_{i}-1}+\cdots+a_{i 1}^{*} s+a_{i 0}^{*}, i=1,2$, are stable monic polynomials such that the high-frequency gain matrix

$$
K_{p}=\lim _{s \rightarrow \infty} \xi_{m}(s) G(s)=\left[\begin{array}{l}
C_{1} A^{\rho_{1}-1} B \\
C_{2} A^{\rho_{2}-1} B
\end{array}\right] \in R^{2 * 2}
$$

is finite and nonsingular, where $\rho_{i}, i=1,2$, are the relative degrees of $\left(C_{i}, A, B\right)$.

\subsection{Adaptive Control Design for Three-Phase PV Inverter without Disturbance}

In parallel with Section 4.3, in this section, we will first consider the output feedback control problem for the three-phase inverter system working without the influence from the grid, which is

$$
\dot{x}(t)=A x(t)+B u(t), y(t)=C x(t),
$$


where $A \in R^{6 \times 6}, B \in R^{6 \times 2}$, and $C \in R^{2 \times 6}$ are unknown constant matrices, with $x(t) \in R^{6}, y(t) \in R^{2}$ available for measurement. The physical meaning of this statement is that the inverter system works isolated from the grid, which can be considered as the operation situation before the inverter is connected to the grid. Solving this control problem is a good start before working on the grid-connected inverter system.

The input-output form of the above system is obtained as

$$
y(t)=G_{0}(s)[u](t)
$$

where $G_{0}=C(s I-A)^{-1} B$.

\subsubsection{Nominal Control Design}

In this subsection, the nominal control design is derived for the inverter system working without grid influence.

Nominal Controller With the knowledge of system parameters, the output feedback output tracking MRAC controller is

$$
u^{*}(t)=\Theta_{1}^{* T} \omega_{1}(t)+\Theta_{2}^{* T} \omega_{2}(t)+\Theta_{20}^{*} y(t)+\Theta_{3}^{*} r(t)
$$

where $\Theta_{1}^{*}, \Theta_{2}^{*} \in R^{2 * 6}, \Theta_{20}^{*}, \Theta_{3} \in R^{2 \times 2}, \omega_{1}(t)=F(s)[u](t), \omega_{2}(t)=F(s)[y](t)$ and $F(s)=\frac{1}{A(s)}$, where $A(s)$ is a monic stable polynomial of degree 2 . 
Parameter Matching Property The parameters $\Theta_{1}^{*}, \Theta_{2}^{*}, \Theta_{20}^{*}$ and $\Theta_{3}^{*}$ for the plant (5.1) is chosen to have the following matching property

$$
I-\Theta_{1}^{* T} F(s)-\Theta_{2}^{* T} F(s) G_{0}(s)-\Theta_{20}^{*} G_{0}(s)=\Theta_{3}^{*} W_{m}^{-1}(s) G_{0}(s),
$$

where $G_{0}(s)=C(s I-A)^{-1} B$, and $F(s)=\frac{1}{A(s)}$ is the stable filter defined above. With this matching property, one can prove that the controller designed in this section satisfies the following theorem.

Theorem 5.1. With parameters $\Theta_{1}^{*}, \Theta_{2}^{*}, \Theta_{20}^{*}$ and $\Theta_{3}^{*}$ satisfying the matching property (5.9), the controller (5.8) ensures that all signals in the closed-loop system are bounded and

$$
\lim _{t \rightarrow \infty}\left(y(t)-y_{m}(t)\right)=0
$$

Proof. Operating $u(t)$ on both side of (5.9), we have

$$
u(t)-\Theta_{1}^{* T} F(s)[u](t)-\Theta_{2}^{* T} F(s) G_{0}(s)[u](t)-\Theta_{20}^{*} G_{0}(s)[u](t)=\Theta_{3}^{*} W_{m}^{-1} G_{0}(s)[u](t) .
$$

Substituting the system description (5.7): $y(t)=G_{0}(s)[u](t)$, we have

$$
u(t)-\Theta_{1}^{* T} F(s)[u](t)-\Theta_{2}^{* T} F(s)[y](t)-\Theta_{20}^{*}[y](t)-\Theta_{3}^{*} W_{m}^{-1}[y](t)=0 .
$$

Applying the output feedback controller defined in (5.8) to the equation above, we have

$$
\Theta_{3}^{*} W_{m}(s)^{-1}\left[y-y_{m}\right](t)=0
$$

Since $W_{m}(s)^{-1}$ is Hurwitz, $\Theta_{3}=K_{p}^{-1}$. We have $y(t) \in L^{\infty}$ and

$$
\lim _{t \rightarrow \infty}\left(y(t)-y_{m}(t)\right)=0
$$


With the satisfaction of the theorem above, the nominal controller designed in this subsection can achieve the desired tracking.

\subsubsection{Output Feedback Adaptive Control design}

Based on the nominal controller, in this subsection an output feedback adaptive controller is derived for the three-phase PV inverter with unknown parameters, working isolated from the grid.

Controller Structure The adaptive version of the nonminal controller (5.8) is

$$
u(t)=\Theta_{1}^{T}(t) \omega_{1}(t)+\Theta_{2}^{T}(t) \omega_{2}(t)+\Theta_{20}(t) y(t)+\Theta_{3}(t) r(t)
$$

where $\Theta_{1}(t), \Theta_{2}(t), \Theta_{20}(t)$ and $\Theta_{3}(t)$ are estimates of the system parameters $\Theta_{1}^{*}, \Theta_{2}^{*}, \Theta_{20}^{*}$ and $\Theta_{3}^{*}$.

Tracking Error Equation From (5.12), we can obtain the following equation

$$
u(t)-\Theta_{1}^{* T} \omega_{1}(t)-\Theta_{2}^{* T} \omega_{2}(t)-\Theta_{20}^{*} y(t)-\Theta_{3}^{*} r(t)=\Theta_{3}^{*} \xi_{m}(s)[e](t)
$$

where $e(t)$ is the output tracking error $e(t)=y(t)-y_{m}(t)$. It follows that

$$
\xi_{m}(s)[e](t)=\Theta_{3}^{*-1} \tilde{\Theta}^{T}(t) \omega(t)
$$


where we define

$$
\begin{aligned}
\Theta^{*} & =\left[\Theta_{1}^{* T}, \Theta_{2}^{* T}, \Theta_{20}^{*}, \Theta_{3}^{*}\right]^{T} \\
\Theta(t) & =\left[\Theta_{1}(t), \Theta_{2}(t), \Theta_{20}(t), \Theta_{3}(t)\right]^{T} \\
\omega(t) & =\left[\omega_{1}^{T}(t), \omega_{2}^{T}(t), y(t), r(t)\right]^{T} \\
\tilde{\Theta}(t) & =\Theta(t)-\Theta^{*} .
\end{aligned}
$$

Choose the same $h(s)=\frac{1}{f(s)}$ as the one defined in Section 4.4.2. Then define the filtered tracking error as

$$
\bar{e}(t)=\xi_{m}(t) h(s)[e](t) .
$$

Then, we have

$$
\bar{e}(t)=\Theta_{3}^{*-1} h(s)\left[\tilde{\Theta}^{T} \omega\right](t)
$$

Estimation Error Equation Introducing an estimation error

$$
\varepsilon(t)=\bar{e}(t)+\Psi(t) \xi(t)
$$

where $\Psi(t)$ is the estimate of $\Theta_{3}^{*-1}$ and

$$
\xi(t)=\Theta^{T}(t) \zeta(t)-h(s)\left[\Theta^{T} \omega\right](t), \zeta(t)=h(s)[\omega](t) .
$$

Adaptive Laws The adaptive laws for parameter estimates are chosen as

$$
\begin{aligned}
\dot{\Theta}^{T}(t) & =-\frac{\Gamma_{1} \varepsilon(t) \zeta^{T}(t)}{m^{2}(t)} \\
\dot{\Psi}(t) & =-\frac{\Gamma_{2} \varepsilon(t) \xi(t)}{m^{2}(t)}
\end{aligned}
$$

where $m^{2}(t)=1+\zeta^{T}(t) \zeta(t)+\xi^{T}(t) \xi(t), \Gamma_{1}=\Gamma_{1}^{T} \geq 0, \Gamma_{2}=\Gamma_{2}^{T} \geq 0$. 
The above adaptive laws ensure the same parameter estimation properties in Lemma 4.2. We can derive the following theorem.

Theorem 5.2. For the plant (5.6) under assumption 1 and 2 and the reference model (5.4). The adaptive controller (5.15) with the adaptive update law (5.26)-(5.27), all signals in the closed-loop system are bounded and the tracking error $e(t)=y(t)-y_{m}(t)$ satiefies $\lim _{t \rightarrow \infty} e(t)=0$.

Based on this theorem, we have that the adaptive controller designed in this section can achieve desired tracking.

\subsection{Adaptive Control Design for Three-Phase Grid- connected PV Inverter}

In this section, we will first consider the output feedback control problem for the three-phase grid-connected inverter system (5.6), which is

$$
\dot{x}(t)=A x(t)+B u(t)+B_{d} \delta(t), y(t)=C x(t)
$$

where $A \in R^{6 \times 6}, B \in R^{6 \times 2}$, and $C \in R^{2 \times 6}$ are unknown constant matrices, with $x(t) \in R^{6}, y(t) \in R^{2}$ are some available for measurement.

The input-output form of the above system is obtained as

$$
y(t)=G_{0}(s)[u](t)+\bar{y}(t)
$$

where $G_{0}=C(s I-A)^{-1} B$ and $\bar{y}(t)=G_{d}(s) \delta(t)=C(s I-A)^{-1} B_{d} \delta(t)$ is the output for the disturbance. Note that $G_{0}(s)[u](t) \triangleq \mathcal{L}^{-1}\left[G_{0}(s) u(s)\right]$. 


\subsubsection{Nominal Control Design}

Before starting the adaptive control design, the case where all the system parameters are known, which is the nominal control case, is considered.

Nominal Controller Structure With the knowledge of system parameters, the output feedback output tracking MRAC controller is

$$
u^{*}(t)=\Theta_{1}^{* T} \omega_{1}(t)+\Theta_{2}^{* T} \omega_{2}(t)+\Theta_{20}^{*} y(t)+\Theta_{3}^{*} r(t)+\Theta_{4}^{*}(t)
$$

where $\Theta_{1}^{*}, \Theta_{2}^{*} \in R^{2 \times 6}, \Theta_{20}^{*}, \Theta_{3} \in R^{2 \times 2}$ and $\Theta_{4}^{*}(t) \in R^{2}$ is used to cancel the effects of the disturbance $\delta(t), \omega_{1}(t)=F(s)[u](t), \omega_{2}(t)=F(s)[y](t), F(s)=\frac{1}{A(s)}$, where $A(s)$ is a monic stable polynomial of degree 2 .

Matching Property The parameters $\Theta_{1}^{*}, \Theta_{2}^{*}, \Theta_{20}^{*}$ and $\Theta_{3}^{*}$ for the plant (5.6) are chosen to satisfy the matching property below

$$
I-\Theta_{1}^{* T} F(s)-\Theta_{2}^{* T} F(s) G_{0}(s)-\Theta_{20}^{*} G_{0}(s)=\Theta_{3}^{*} W_{m}^{-1}(s) G_{0}(s)
$$

where $G_{0}(s)=C(s I-A)^{-1} B$, and $F(s)=\frac{1}{A(s)}$ is the stable filter defined above.

With this matching property, substituting the controller to the plant (5.6), the closed-loop system becomes

$$
\begin{aligned}
y(t) & =G_{0}(s)\left[\Theta_{3}^{*} W_{m}(s) G_{0}(s)\right]^{-1}\left[\Theta_{2}^{* T} F(s) \bar{y}(t)+\Theta_{20}^{*} \bar{y}(t)+\Theta_{3}^{*} r(t)+\Theta_{4}^{*}(t)\right]+\bar{y}(t) \\
& =W_{m}(s)[r](t)+f_{p}(t),
\end{aligned}
$$


where $f_{p}(t)$ is the effect of the disturbance

$$
f_{p}(t)=W_{m}(s) \Theta_{3}^{*-1}\left[\Theta_{2}^{* T} F(s)[\bar{y}](t)+\Theta_{20}^{*} \bar{y}(t)+\Theta_{3}^{*} W_{m}^{-1}(s)[\bar{y}](t)+\Theta_{4}^{*}(t)\right]
$$

To cancel the effect of the disturbance $f_{p}(t)$, we can choose $\Theta_{4}^{*}(t)$ as

$$
\Theta_{4}^{*}(t)=-\Theta_{2}^{* T} F(s)[\bar{y}](t)-\Theta_{20}^{*} \bar{y}(t)-\Theta_{3}^{*} W_{m}^{-1}(s)[\bar{y}](t)
$$

With this choice of $\Theta_{4}^{*}(t)$ we can make $f_{p}(t)=0$.

Parameterlization for $\Theta_{4}^{*}(t)$ Rewrite (5.35) as

$$
\Theta_{4}^{*}(t)=-\Theta_{2}^{* T} F(s) G_{d}(s)[d](t)-\Theta_{20}^{*} G_{d}(s)[d](t)-\Theta_{3}^{*} W_{m}^{-1}(s) G_{d}(s)[d](t) .
$$

Notice that in the term $\Theta_{3}^{*} W_{m}^{-1}(s) G_{d}(s)[d](t), W_{m}^{-1}(s) G_{d}(s)$ is not proper. Hence, to parameterlize $\Theta_{4}^{*}(t)$, the knowledge of $\dot{\delta}(t)$ is needed. With the proof in [4], we have

$$
\begin{aligned}
\Theta_{4}^{*}(t) & =P_{\alpha 1}(s) F(s)[d](t)+Q(s)[d](t) \\
& =P_{\alpha 1}(s) F(s)[d](t)+P_{\alpha 2}(s) F(s)[d](t)+P_{\alpha 3}(s) F(s)[\dot{d}](t) \\
& =P_{\alpha}(s) F(s)\left[\Phi^{*} f+\tilde{\Phi}^{*} f\right](t) \\
& =P_{\alpha}(s) F(s) \bar{\Phi}^{*} f(t),
\end{aligned}
$$

where $P_{\alpha i}(s) F(s), i=1,2,3$ is proper, $Q(s)$ has relative degree 1 and $P_{\alpha} F(s)$ is proper. The last two equation is due to the fact that the derivitive of $\delta(t)$ share the same components $(f(t))$ with $\delta(t)$.

With the fact that $P_{\alpha}(s) F(s)$ is proper. The choice of $\Theta_{4}^{*}(t)$ in (5.40) can be 
rewritten as

$$
\Theta_{4}^{*}(t)=\Theta_{5}^{* T} \omega_{3}(t)
$$

where the parameterized variables $\Theta_{5}^{* T}$ and $\omega_{3}(t)$ are

$$
\begin{aligned}
\Theta_{5}^{* T} & =\left(\Theta_{\alpha} \bar{\Phi}^{* T}\right)^{T} \in R^{5 \times 2} \\
\omega_{3}(t) & =\left[\left(I_{4} F(s)[f](t)\right)^{T}, f(t)^{T}\right]^{T} \in R^{5 \times 2},
\end{aligned}
$$

where $I_{4}$ is the identical matrix. With the derivation above, we can propose the following proposition.

Proposition 5.1. The disturbance signal $\delta(t)$ in (4.63) being the summation of sinusoidal signals, the disturbance related control parameter $\Theta_{4}^{*}(t)=P_{\alpha 1}(s) F(s)[d](t)+$ $Q(s)[d](t)$ can be parameterized to the form

$$
\Theta_{4}^{*}(t)=\Theta_{5}^{* T} \omega_{3}(t)
$$

where $\omega_{3}(t)=\left[\left(I_{4} F(s)[f](t)\right)^{T}, f(t)^{T}\right]^{T}$ and $f(t)$ contains all the fundamental sinusoidal signals.

Next, the adaptive version of the control scheme is studied for systems without the knowledge of system and disturbance parameters.

\subsubsection{Output Feedback Adaptive Control design}

Based on the nominal controller obtained from the previous section, in this subsection the output feedback model reference adaptive control scheme for the three phase PV inverter working in grid-connection is proposed. 
Controller Structure The adaptive controller has the same structure as the nonminal controller (5.8)

$$
u(t)=\Theta_{1}^{T}(t) \omega_{1}(t)+\Theta_{2}^{T}(t) \omega_{2}(t)+\Theta_{20}(t) y(t)+\Theta_{3}(t) r(t)+\Theta_{4}(t),
$$

where $\Theta_{1}(t), \Theta_{2}(t), \Theta_{20}(t), \Theta_{3}(t)$ and $\Theta_{4}(t)$ are estimates of the system parameter $\Theta_{1}^{*}, \Theta_{2}^{*}, \Theta_{20}^{*}, \Theta_{3}^{*}$ and $\Theta_{4}^{*}(t)$.

Tracking Error Equation From (5.9), we can obtain the following equation

$$
u(t)-\Theta_{1}^{* T} \omega_{1}(t)-\Theta_{2}^{* T} \omega_{2}(t)-\Theta_{20}^{*} y(t)-\Theta_{3}^{*} r(t)=\Theta_{3}^{*} \xi_{m}(s)[e](t),
$$

where $e(t)$ is the output tracking error $e(t)=y(t)-y_{m}(t)$. It follows that

$$
\xi_{m}(s)[e](t)=\Theta_{3}^{*-1} \tilde{\Theta}^{T}(t) \omega(t)
$$

where

$$
\begin{aligned}
\Theta^{*} & =\left[\Theta_{1}^{* T}, \Theta_{2}^{* T}, \Theta_{20}^{*} \Theta_{3}^{*}, \Theta_{5}^{*}\right]^{T}, \\
\Theta(t) & =\left[\Theta_{1}(t), \Theta_{2}(t), \Theta_{20}(t) \Theta_{3}(t), \Theta_{5}(t)\right]^{T}, \\
\omega(t) & =\left[\omega_{1}^{T}(t), \omega_{2}^{T}(t), y(t), r(t) \omega_{3}^{T}(t)\right]^{T}, \\
\tilde{\Theta}(t) & =\Theta(t)-\Theta^{*} .
\end{aligned}
$$

Choose the same filter $h(s)=\frac{1}{f(s)}$ as the one defined in Section 4.4.2. Then define the filtered tracking error as

$$
\bar{e}(t)=\xi_{m}(s) h(s)[e](t) .
$$


Then, we have

$$
\bar{e}(t)=\Theta_{3}^{*-1} h(s)\left[\tilde{\Theta}^{T} \omega\right](t)
$$

Estimation Error Equation Introducing an estimation error

$$
\varepsilon(t)=\bar{e}(t)+\Psi(t) \xi(t)
$$

where $\Psi(t)$ is the estimate of $\Theta_{3}^{*-1}$ and

$$
\xi(t)=\Theta^{T}(t) \zeta(t)-h(s)\left[\Theta^{T} \omega\right](t), \zeta(t)=h(s)[\omega](t) .
$$

Adaptive Laws The adaptive laws for parameter estimates are chosen as

$$
\begin{aligned}
\dot{\Theta}^{T}(t) & =-\frac{\Gamma_{1} \varepsilon(t) \zeta^{T}(t)}{m^{2}(t)} \\
\dot{\Psi}(t) & =-\frac{\Gamma_{2} \varepsilon(t) \xi(t)}{m^{2}(t)}
\end{aligned}
$$

where $m^{2}(t)=1+\zeta^{T}(t) \zeta(t)+\xi^{T}(t) \xi(t), \Gamma_{1}=\Gamma_{1}^{T} \geq 0, \Gamma_{2}=\Gamma_{2}^{T} \geq 0$.

The above adaptive laws ensure the same parameter estimation properties in Lemma 3.2. And we can derive the theorem below

Theorem 5.3. For the plant (5.6) under assumption 1 and 2 and the reference model (5.4). The adaptive controller (5.45) with the adaptive update law (5.56)-(5.57), all signals in the closed-loop system are bounded and the tracking error $e(t)=y(t)-y_{m}(t)$ satiefies $\lim _{t \rightarrow \infty} e(t)=0$.

With this theorem, we have that the adaptive controller designed in this section can achieve the desired tracking. 


\subsection{Simulation Results}

In this section, the simulation results are presented for output feedback output tracking control schemes for inverter system. Firstly the results for the adaptive controller inverter working without grid influence are introduced then the results for the inverter working grid-connected are proposed.

\subsubsection{Inverter System Operating without Disturbance}

For the inverter system working without grid influence, the system state space equation is

$$
\dot{x}(t)=A x(t)+B u(t), y(t)=C x(t),
$$

where

Table 5.1: Simulation study parameter [17], [30]

\begin{tabular}{|l|l|l|}
\hline Parameter & Unit & Value \\
\hline Inductor $L_{f}$ & $(\mathrm{mH})$ & 0.9 \\
\hline Capacitor $C$ & $(\mu \mathrm{F})$ & 30 \\
\hline Inductor $L_{g}$ & $(\mathrm{mH})$ & 0.54 \\
\hline Resistance $R_{f}$ & $(\mathrm{~m} \Omega)$ & 0.05 \\
\hline Resistance $R_{g}$ & $(\mathrm{~m} \Omega)$ & 0.03 \\
\hline Resistance $R_{c}$ & $(\mathrm{~m} \Omega)$ & 0.05 \\
\hline Line-to-line grid voltage $U_{a, b, c}$ & $(\mathrm{~V})$ & 380 \\
\hline Grid frequency $\omega$ & $(\mathrm{rad} / \mathrm{s})$ & 314.15 \\
\hline Input voltage $U_{P V}$ & $(\mathrm{~V})$ & $750-820$ \\
\hline
\end{tabular}




$$
\begin{aligned}
& x(t)=\left[\begin{array}{llllll}
I_{i d} & I_{i q} & I_{g d} & I_{g q} & U_{P V} & U_{q c}
\end{array}\right]^{T}, u(t)=\left[\begin{array}{ll}
d_{d} & d_{q}
\end{array}\right], \\
& A=\left[\begin{array}{cccccc}
-88.89 & 314.16 & 33.33 & 0 & -1111.11 & 0 \\
-314.16 & -88.89 & 0 & 33.33 & 0 & -1111.11 \\
111.11 & 0 & -55.56 & 314.16 & 1851.85 & 0 \\
0 & 111.11 & -314.16 & -55.56 & 0 & 1851.85 \\
33333.33 & 0 & -33333.33 & 0 & 0 & 314.16 \\
0 & 33333.33 & 0 & -33333.33 & -314.16 & 0
\end{array}\right] \text {, } \\
& B=\left[\begin{array}{cccccc}
5.13 \times 10^{5} & 0 & 0 & 0 & 0 & 0 \\
0 & 5.13 \times 10^{5} & 0 & 0 & 0 & 0
\end{array}\right], \\
& C=\left[\begin{array}{llllll}
0 & 0 & 1 & 0 & 0 & 0 \\
0 & 0 & 0 & 1 & 0 & 0
\end{array}\right] \text {. }
\end{aligned}
$$

These nominal values are obtained by applying the system parameters in Table 5.1 to the system matrix $(A, B, C)$ in (3.11)-(3.13).

This linear model can be treated as a test bed to do simulations systematically to verify the effectiveness of our proposed adaptive control scheme.

Adaptive Control design For the inverter system (4.104), it can be verified that the transfer matrix $G(s)=C(s I-A)^{-1} B$ has stable zeros: $z_{1}=(-5.56-j 0.0031) \times$ $10^{5}, z_{2}=(-5.56+k 0.0031) \times 10^{5}$. Also, this transfer matrix is strictly proper and full rank. Choosing the interactor matrix as $\xi_{m}(s)=\operatorname{diag}\left\{(s+1)^{2},(s+1)^{2}\right\}$, such 
that the high-frequency gain matrix is

$$
K_{p}=\lim _{s \rightarrow \infty} \xi_{m}(s) G(s)=\left[\begin{array}{cc}
5.7022 \times 10^{7} & 0 \\
0 & 5.7022 \times 10^{7}
\end{array}\right]
$$

which is positive finite and nonsingular. Then the reference model is chosen as

$$
W_{m}(s)=\xi_{m}^{-1}(s)=\operatorname{diag}\left\{\frac{1}{(s+1)^{2}}, \frac{1}{(s+1)^{2}}\right\}
$$

Choosing $f(s)=(s+1)^{2}$ and $h(s)=\frac{1}{(s+1)^{2}}$.

Simulation Results Choose the initial control parameters 0.8 times of their true nominal values. Choose $\Gamma_{1}=\Gamma_{2}=0.1 I$. There two cases of simulation results. In Case 1 , the system tracks a constant output $y_{m}=\left[I_{g d}^{*}, I_{g q}^{*}\right]^{T}$, where $I_{g d}^{*}=\frac{2 P^{*}}{3 U_{d} N}=$ $17 A, I_{g q}=0$. In Case 2 , the system tracks $y_{m}(t)$ which initially has the same value as in Case 1 and then increases as time goes.

Case 1: Choose $r(t)=[17,0]^{T}, y_{m}(0)=[17,0]^{T}$. In this way, $y_{m}$ is just constant signal. Figure 5.1b shows the output current and the tracking error in the d-q axis. We can see that the system achieved desired tracking after 0.1 seconds. Figure $5.1 \mathrm{a}$ shows the three-phase output current, from which we can see that the system achieved the desired output tracking.

Case 2: Choose $r(t)=[17,0]^{T}, t \leq 1, r(t)=[20,0]^{T}, t>1$, this way $y_{m}$ changes smoothly. Figure 5.2a shows the desired tracking signal $y_{m}(t)$ which is a slowly increasing signal. Figure 5.2b show the output current and the tracking error in the $\mathrm{d}-\mathrm{q}$ axis. This result shows that if $y_{m}(t)$ is a time variant signal, the adaptive controller designed for this situation can still achieve tracking. This is something that a classic controller cannot do. 


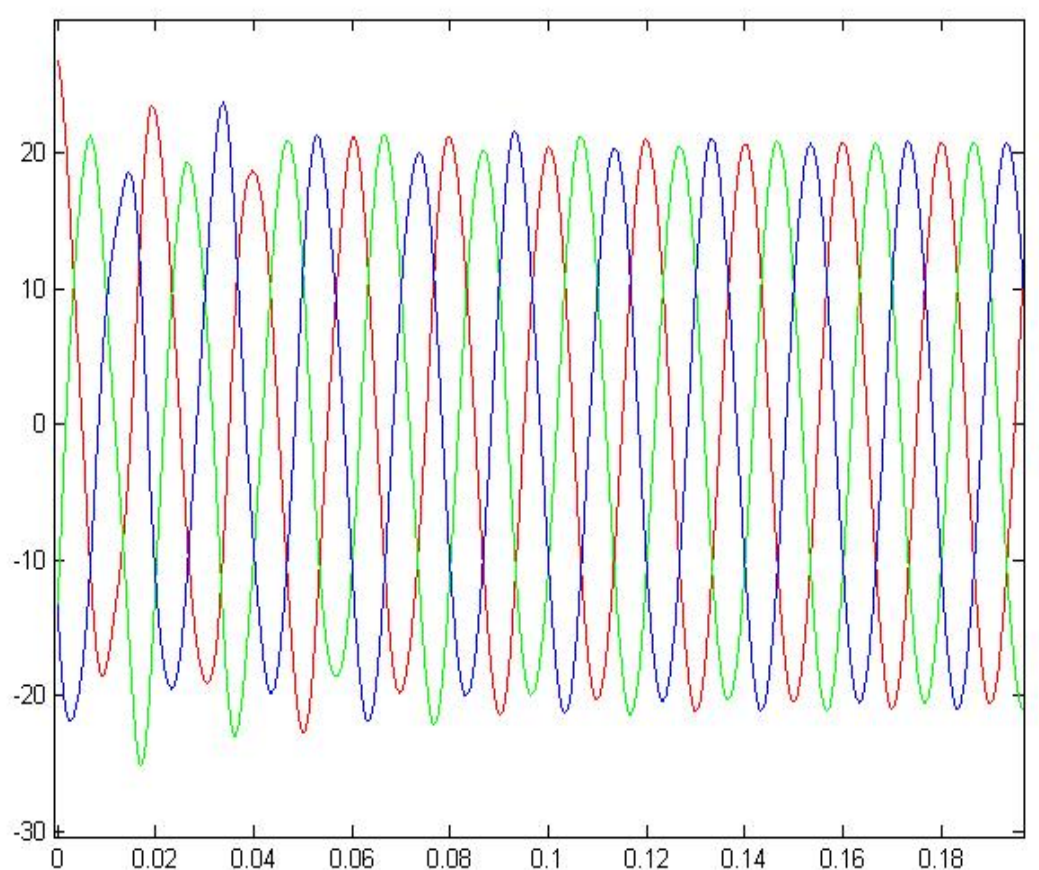

(a) The output current in the a-b-c reference frame.
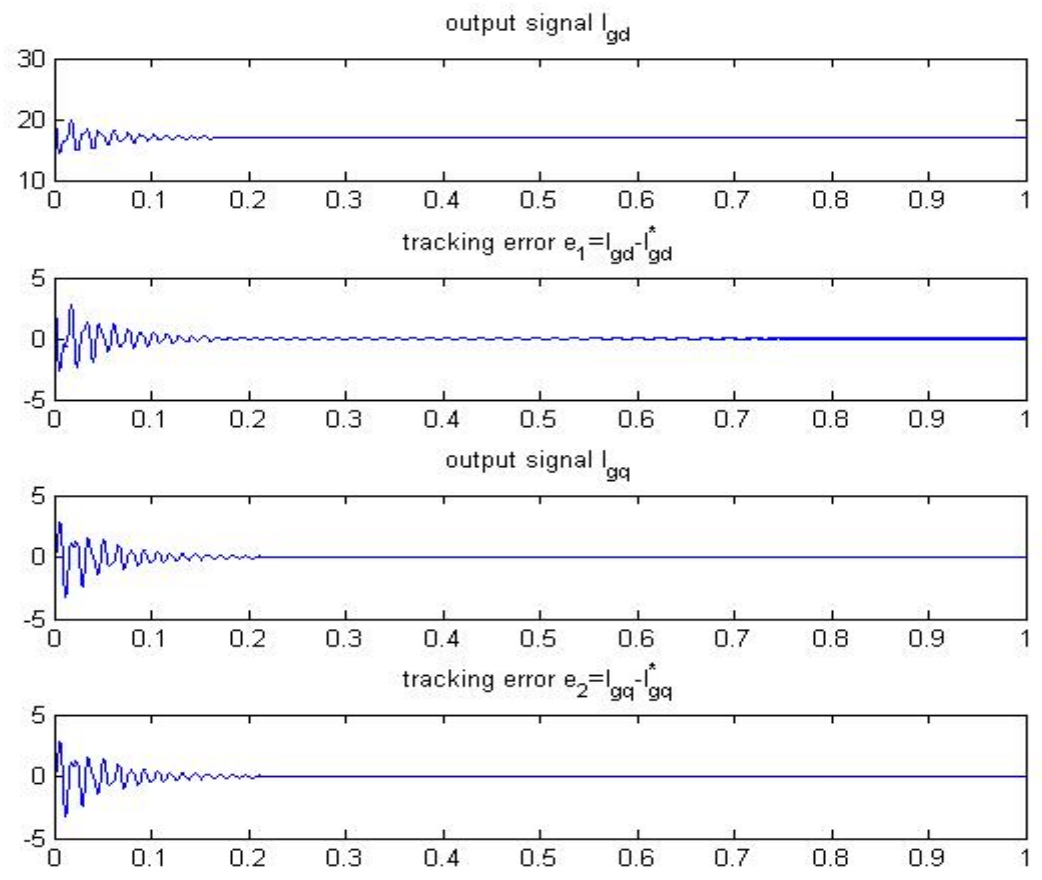

(b) The output current and tracking error of $I_{g d}, I_{g q}$.

Figure 5.1: System response for Case 1. 

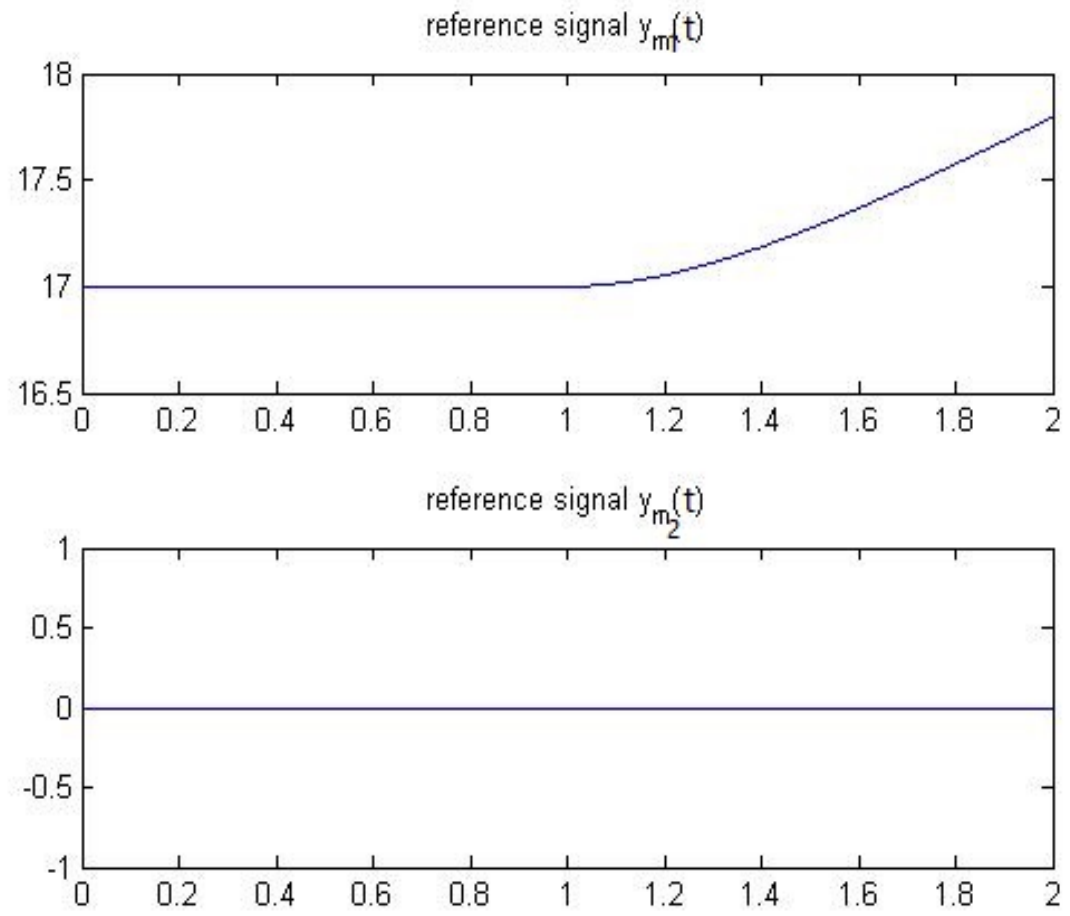

(a) The reference signal $y_{m}(t)$.
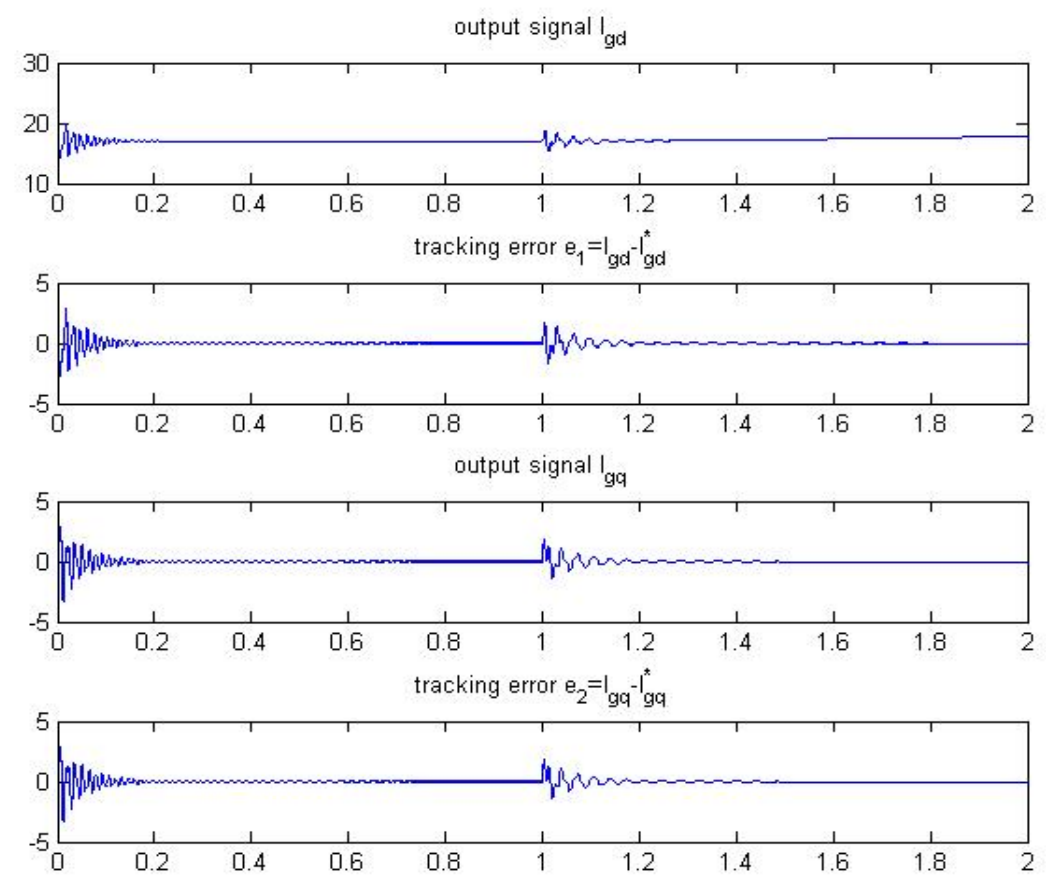

(b) The output current and tracking error of $I_{g d}, I_{g q}$.

Figure 5.2: System response for Case 2. 


\subsubsection{Inverter System Operating in Grid Connected Mode}

For the inverter system working grid-connected, the system state space equation is

$$
\dot{x}(t)=A x(t)+B u(t)+B_{d} \delta(t), y(t)=C x(t),
$$

where $A, B, C$ and the definitions of $x(t), u(t)$ are stated in Section 4.5.2. and

$$
\begin{aligned}
B_{d} & =\left[\begin{array}{cccccc}
0 & 0 & -1.28 \times 10^{4} & 0 & 0 & 0 \\
0 & 0 & 0 & -1.28 \times 10^{4} & 0 & 0
\end{array}\right]^{T}, \\
\delta(t) & =\left[\begin{array}{l}
U_{d N}(t) \\
U_{q N}(t)
\end{array}\right] .
\end{aligned}
$$

Adaptive Control Design For the inverter system (4.104), it can be verified that the transfer matrix $G(s)=C(s I-A)^{-1} B$ has stable zeros: $z_{1}=(-5.56-j 0.0031) \times$ $10^{5}, z_{2}=(-5.56+j 0.0031) \times 10^{5}$. Also, this transfer matrix is strictly proper and full rank. Choosing the interactor matrix as $\xi_{m}(s)=\operatorname{diag}\left\{(s+1)^{2},(s+1)^{2}\right\}$, such that the high-frequency gain matrix is

$$
K_{p}=\lim _{s \rightarrow \infty} \xi_{m}(s) G(s)=\left[\begin{array}{cc}
5.7022 \times 10^{7} & 0 \\
0 & 5.7022 \times 10^{7}
\end{array}\right]
$$

being positive finite and nonsingular and the matrix

$$
K_{d}=\lim _{s \rightarrow \infty} \xi_{m}(s) G_{d}(s)=\left[\begin{array}{cc}
-1.8519 \times 10^{3} & 0 \\
0 & -1.8519 \times 10^{3}
\end{array}\right]
$$


is finite. Then the reference model is chosen as

$$
W_{m}(s)=\xi_{m}^{-1}(s)=\operatorname{diag}\left\{\frac{1}{(s+1)^{2}}, \frac{1}{(s+1)^{2}}\right\} .
$$

We also choose $f(s)=(s+1)^{2}$ and $h(s)=\frac{1}{(s+1)^{2}}$ for MRAC design.

Simulation Results Choose $\Gamma_{1}=\Gamma_{2}=0.1 I$. There are three cases of simulation results presented. Case 3 shows the inverter system working in a standard grid which means $\delta(t)=[310,0]^{T}$, for this case the ability of the adaptive controller to work normally in the grid is tested (with initial parameters equal to the true nominal parameters). Case 4 shows the inverter working the standard grid with system parameters unknown (choosing the initial control parameters 0.8 times of their true nominal values), this will test the ability to deal with system uncertainties for the adaptive controller. Case 5 shows the inverter working in a polluted grid, i.e., the grid side voltage have some harmonic components. This case will show the disturbance rejection ability of the controller to work under unknown harmonic disturbance. Since for case 3, our preliminary concern is to test the ability of our control system to handle the disturbance rather than other uncertainties in the system, the initial control parameters are chosen as the true values.

Case 3: Choose $r(t)=[17,0]^{T}, y_{m}(0)=[17,0]^{T}, \delta(t)=[310,0]^{T}$. Figure 5.3b shows the output current and the tracking error in the d-q axis. Figure 5.3a shows the three phase output current, from which we can see that the system achieved the desired output current. Figure 5.4 shows the FFT analysis of the output current, where the sampling interval for the FFT analysis is $5 \times 10^{-5} \mathrm{~s}$. We can see that by using our control method, the THD is $0.01 \%$ in this case. 


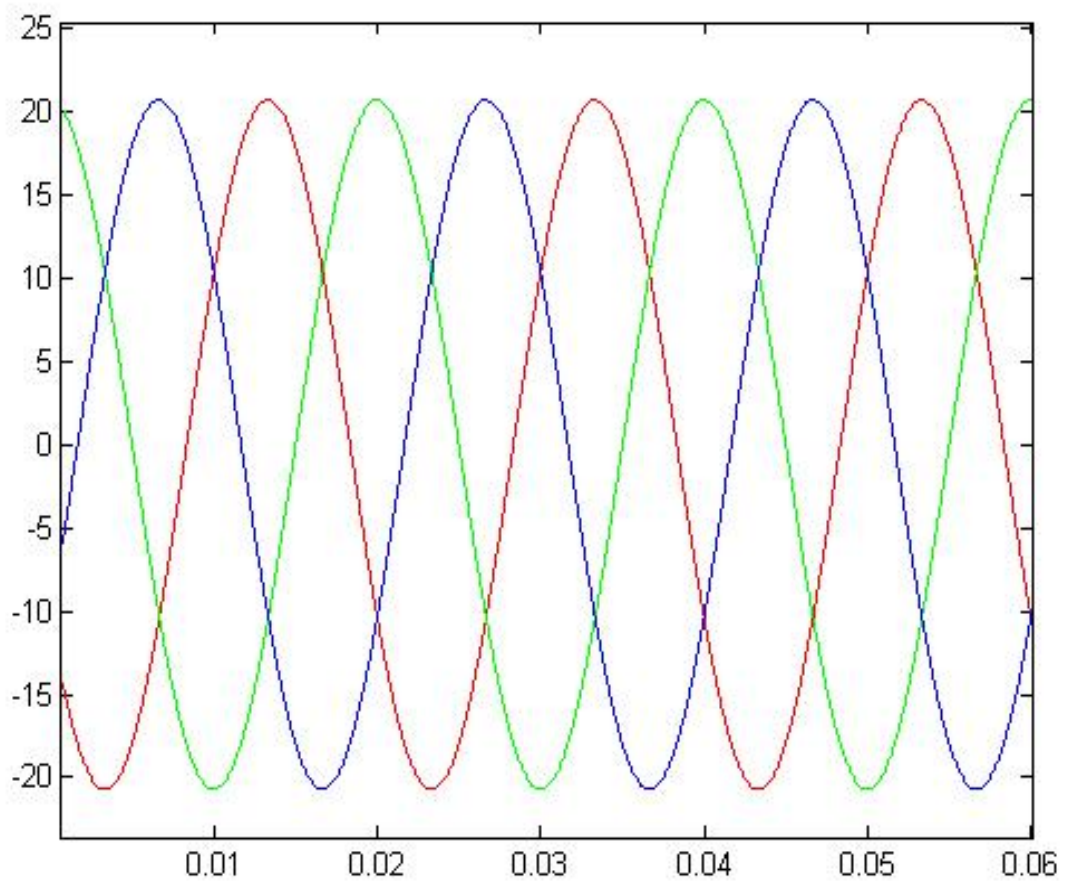

(a) The output current in a-b-c reference.
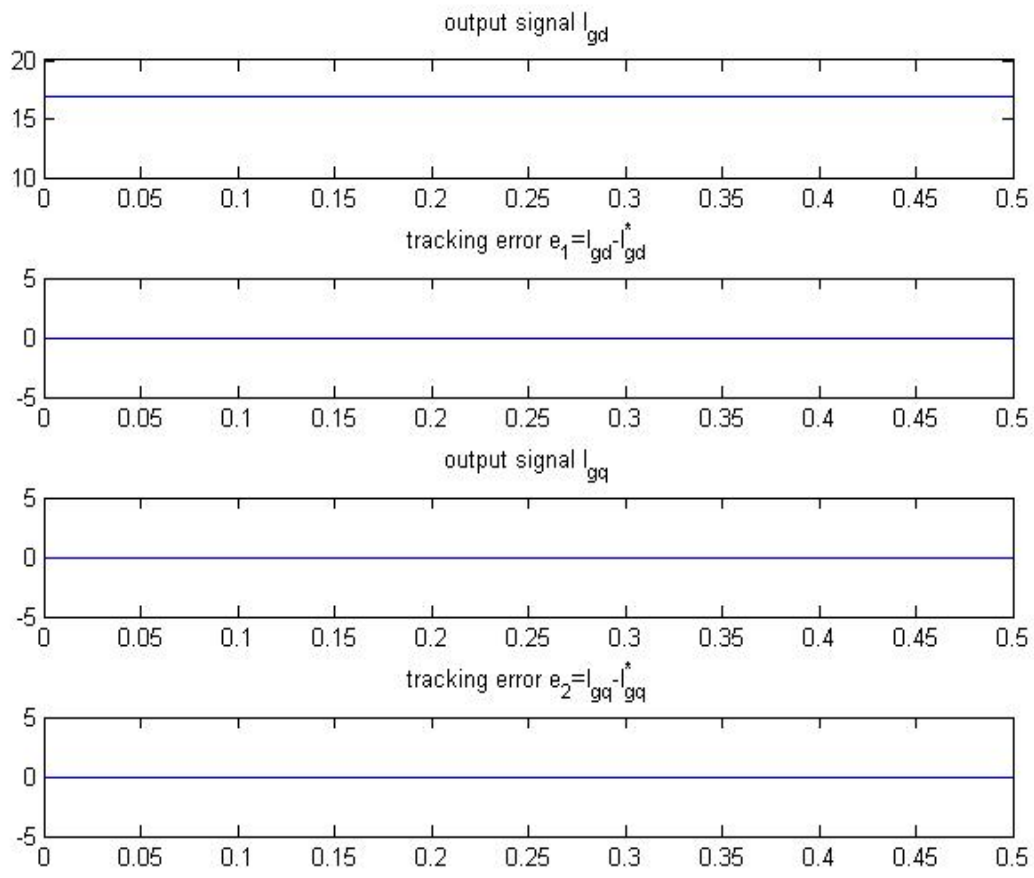

(b) The output current and tracking error of $I_{g d}, I_{g q}$.

Figure 5.3: System response for Case 3. 

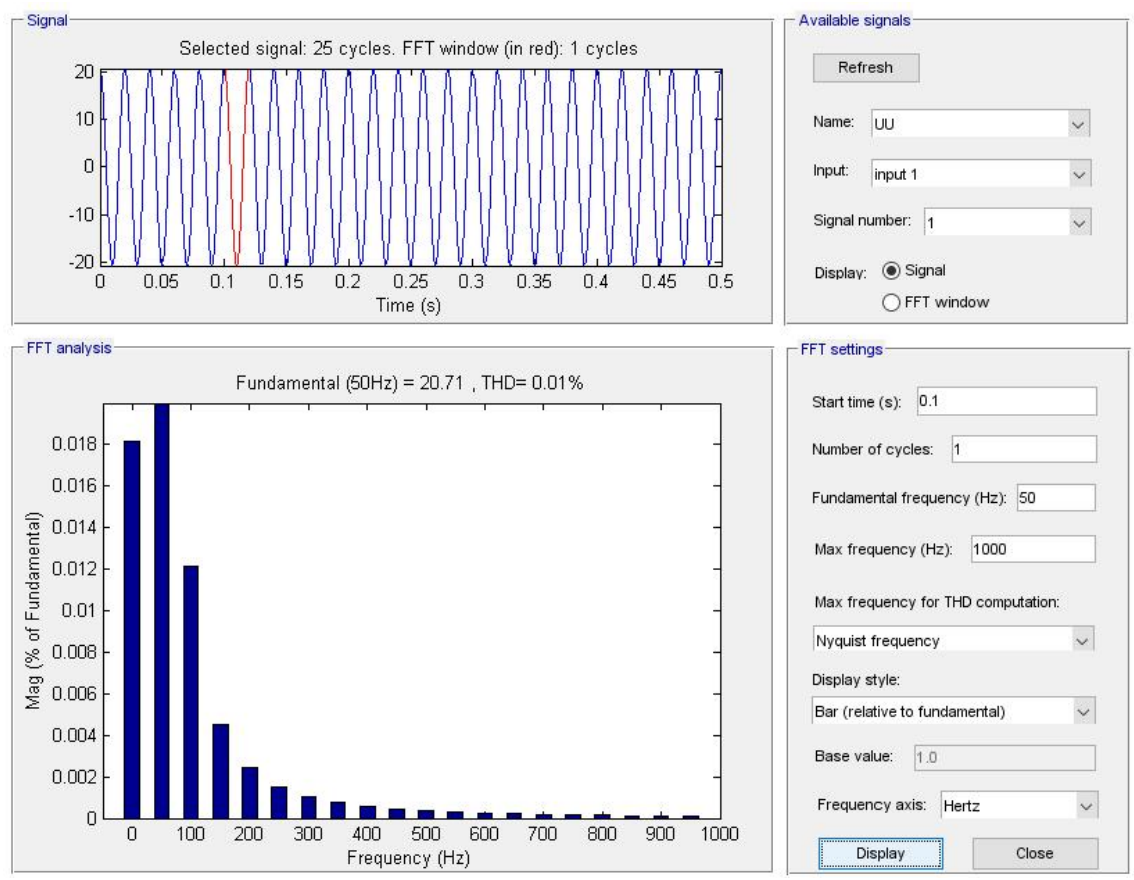

Figure 5.4: FFT analysis for the output current $I_{g a}$ for Case 3.

Case 4: Choose $r(t)=[17,0]^{T}, y_{m}(0)=[17,0]^{T}, \delta(t)=[310,0]^{T}$, all initial control parameters are 0.9 times of their true nominal values. Figure 5.5 shows the output current and the tracking error in the d-q axis, from which we can see that if uncertainties appears in the system, an output feedback adaptive controller have the ability to achieve desired control objective, however the convergence rate is slower than state feedback controller. The system takes 10 seconds to track the desired reference signal. This slow convergence rate is due to the higher order of the output feedback controller. The calculations are more complicated than the state feedback controller. 

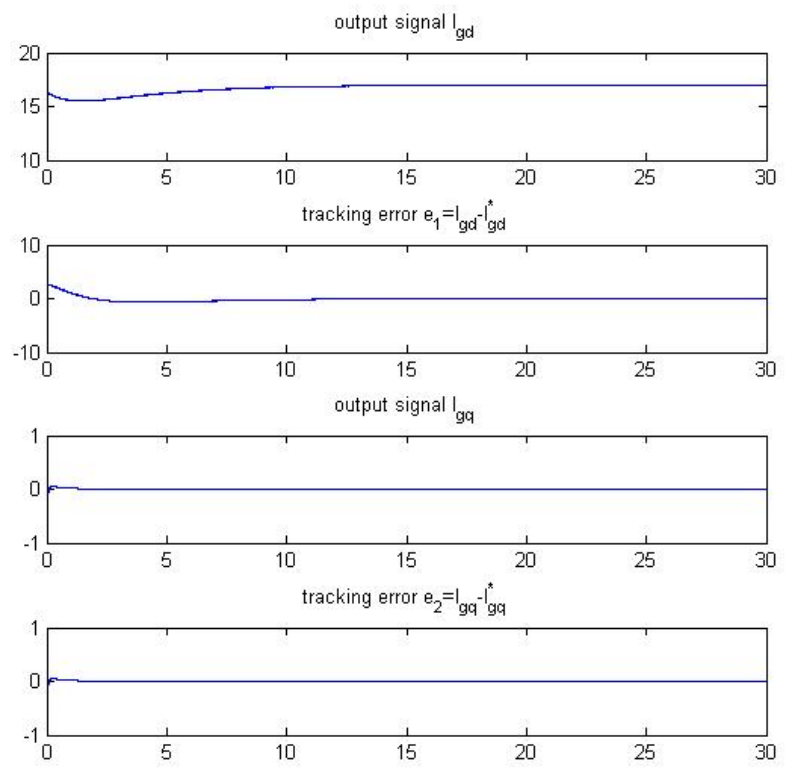

Figure 5.5: The output current and tracking error of $I_{g d}, I_{g q}$ for Case 4.

Case 5 Choose $r(t)=[17,0]^{T}, y_{m}(0)=[17,0]^{T}$. The polluted grid is considered as the disturbance

$$
\delta(t)=\left[\begin{array}{c}
310+10 \cos (6 \omega t)+10 \sin (6 \omega t)+5 \cos (12 \omega t)+5 \sin (12 \omega t) \\
10 \cos (6 \omega t)+10 \sin (6 \omega t)+5 \cos (12 \omega t)+5 \sin (12 \omega t)
\end{array}\right]
$$

The THD of the grid side voltage is $6.2 \%$. Figure $5.6 \mathrm{~b}$ shows the output current and the tracking error in the d-q axis. Figure 5.6a shows the three-phase output current. We can see that the system achieved the desired output current. Figure 5.7 shows the FFT analysis of the output current with the sampling interval $5 \times 10^{-5} \mathrm{~s}$. We can see that by using our control method, the THD in this case is $3.41 \%$, less than the grid side voltage THD. 


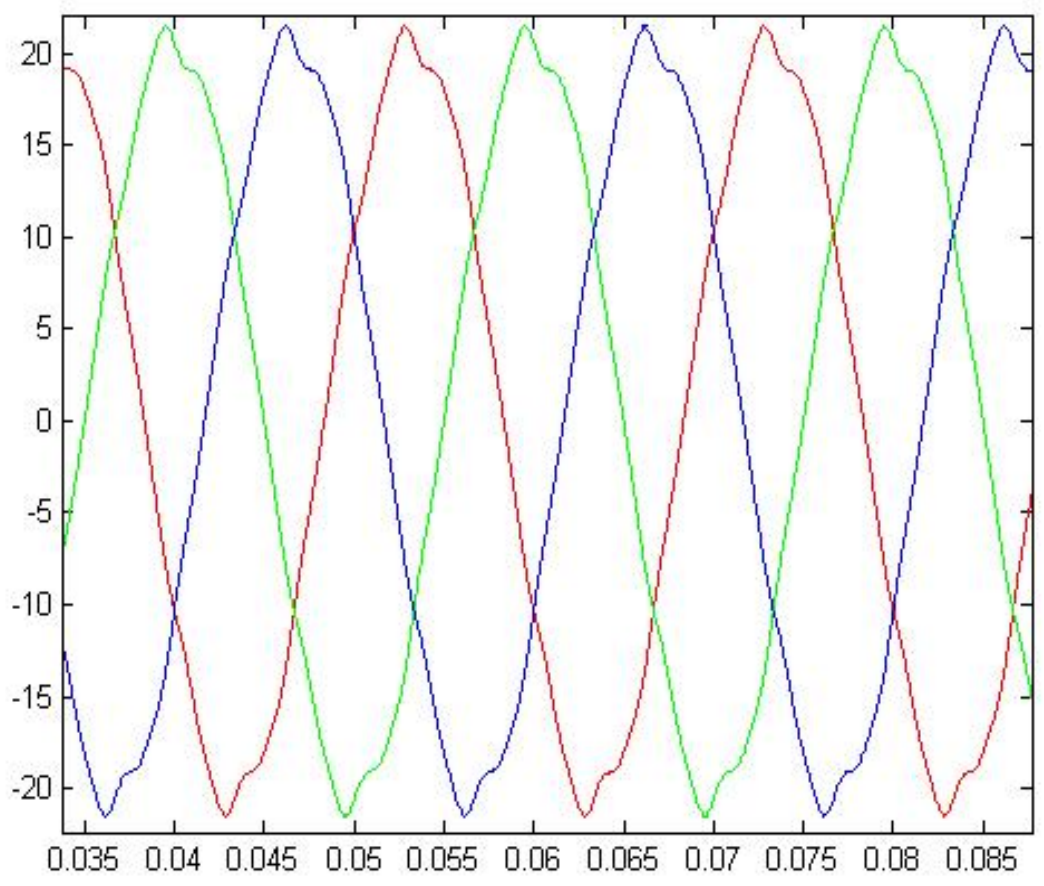

(a) The output current in the a-b-c reference frame.
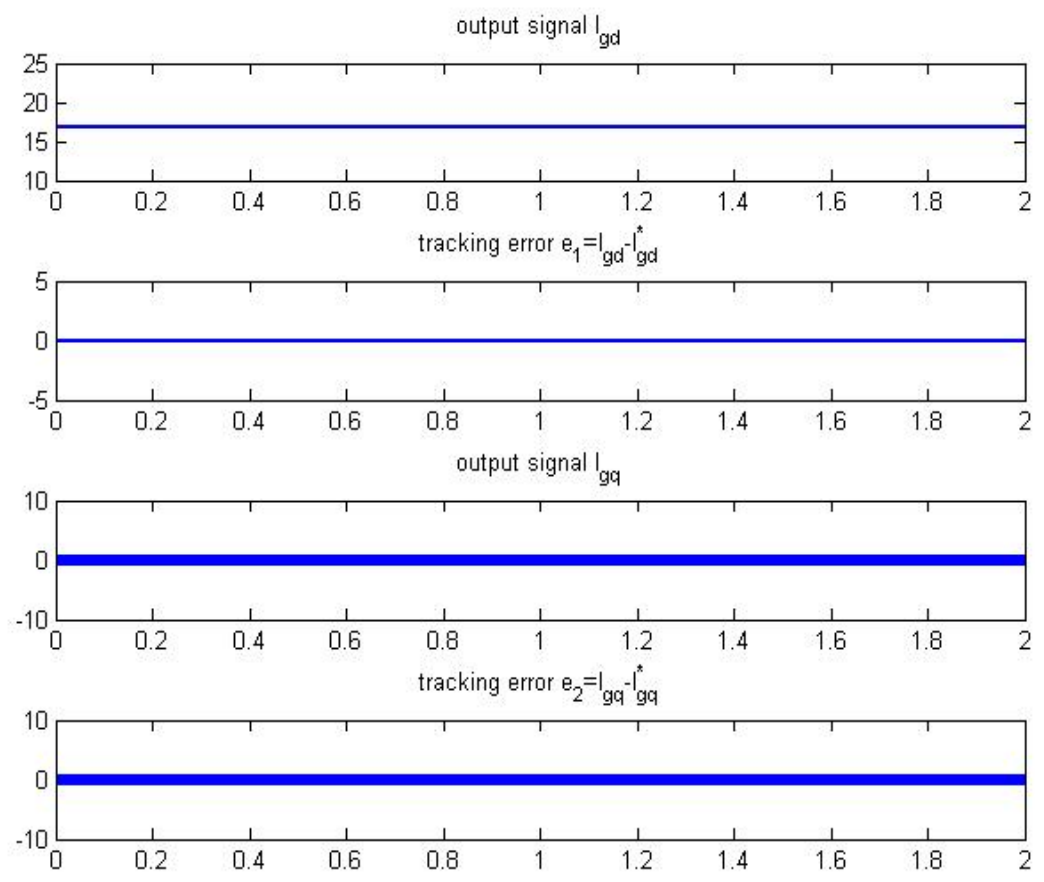

(b) The output current and tracking error of $I_{g d}, I_{g q}$.

Figure 5.6: System response for Case 5. 

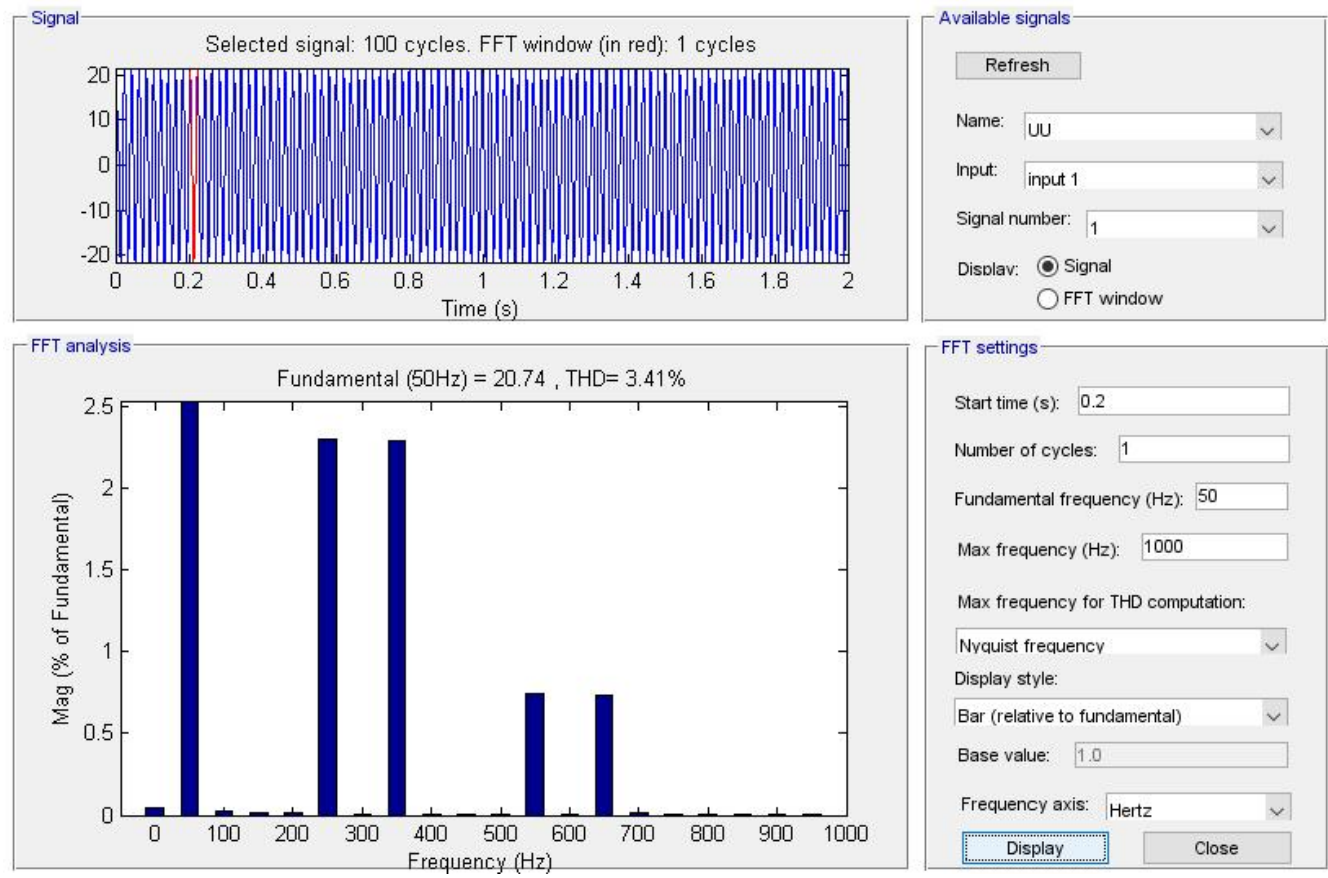

Figure 5.7: FFT analysis for the output current $I_{g a}$ for Case 5.

\subsection{Summary}

In this chapter, the output feedback adaptive control schemes for the three-phase inverter system working with and without grid-connection are designed. Simulation results are presented to verify our designs. From the simulation result, we can conclude that the control objective is achieved. Comparing with the state feedback adaptive controller in Chapter 4, in the case where there exist no disturbance, both controllers show a similar system performance. In the grid-connected mode, however, the output controller system track the reference signal very slowly. The system performance is not as good as the cases for the state feedback controller. Although output feedback adaptive controller can achieve the adaptive control design by only knowing the output signals of the inverter system, the design parameters are twice as much as the state feedback controller, and the controller structure is more compli- 
cated. With further experiments and changes on some control parameters, a better system performance for the output feedback adaptive controller might be obtained. 


\section{Chapter 6}

\section{Conclusions and Future Topics}

This chapter is a summary of this thesis. The main results of this thesis are stated. Then some future works after this research are discussed.

\subsection{Summary and Conclusions}

In this thesis, the motivations for committing to this research topic are given, and the state-of-the-art research related to the inverter control problem is discussed. Following this, the basics of classical control theory, as well as adaptive control theory, are presented, and the approach to modeling for the grid-connected inverter system is presented. With the analysis of the needs for the grid connected inverter system, the control objective is stated. Then both state feedback and output feedback output tracking MRAC schemes for this control objective are derived. Simulation results show that both schemes achieve the control objective.

From this research, we can draw the following conclusions:

(i) The control objective for the grid-connected PV inverter is achieved by both control schemes. 
(ii) Comparing with a classical gain fixed control method, the adaptive controllers, designed in this thesis have the ability to deal with uncertainties of the system parameters as well as grid side influence (such as a change of the grid voltage or the grid voltage with some harmonics).

(iii) Although both controllers meet the control objective,seen from the simulation results, the output feedback controller has a slower system response than the state feedback controller. This is due to the higher order complexity of the output feedback controller. Some further tests for the controller parameters to make the output feedback controller to achieve better performance are needed before it can be used in reality.

\subsection{Future Research Topics}

The adaptive control for the three phase PV inverter with grid-connection, as discussed and analyzed in this thesis, is a new research topic. There still have many related issues. In this section, several possible extensions to our research are introduced.

(i) The control problem for the inverter system is a practice problem, and the control method should be reliable and tested in practical before applied to the power supply company to avoid damage to the grid. In the further research, experiments should be set up to verify the theoretical results in this thesis.

(ii) So far for the disturbance rejection part of this research, we consider most disturbance and unwanted high order harmonics coming from the grid side. To simplify the design, some nonlinearities of the system are ignored, and there might exist some other generation of higher order harmonics. In the future 
work, the effect of these modules in the system may be discussed.

(iii) This research theoretically proved that the adaptive control method can be used for the inverter control problem and can be used to eliminate harmonics. The simulation result shows that the output current of the inverter meets the THD standard in IEEE 1547 standard. However, the THD is not as small as some other research. As a result, an optimal design of adaptive control is needed to achieve better performance.

(iv) Theoretically, the result for this research can be used for other polluted grid such as the three phase unbalanced grid or the frequency change of the grid. For the three phase unbalanced grid, the inverter system should have the function to compensate some distortion of the grid. As a result, the further derivation of the reference current signal is needed. As for the frequency varying case, an adaptive phase lock module is needed.

(v) Although simulation results have proved that the control objective for the threephase inverter system is achieved, a large amount of calculation is needed. The reduced order adaptive control method will be studied in the future to improve this situation. 


\section{Bibliography}

[1] M. Munsell, US Solar Market Grows 95\% in 2016, Smashes Records, Greentech Media, February 15, 2017.

[2] D. R. Baker, "Brown signs climate law mandating 50\% renewable power by 2030", San Francisco Chronicle, October 7, 2015.

[3] R. Teodorescu, F.Blaabjerg, U. Borup and M. Liserre, "A New Control Structure for Grid-Connected LCL PV Inverters with Zero Steady-State Error and Selective Harmonic Compensation," Applied Power Electronics Conference and Exposition, 2004. APEC'04. Nineteenth Annual IEEE, pp. 22-26, February 2004.

[4] Y. Liu and G. Tao "An Adaptive Disturbance Rejection Algorithm for MIMO Systems with An Aircraft Flight Control Application," Proceedings of 2007 AIAA Guidance, Navigation and control congerence, pp. 1-17, South Carolina, 2007.

[5] J. C. Vasquez, J. M. Guerrero, A. Luna, P. Rodrguez and R. Teodorescu, "Adaptive Droop Control Applied to Voltage-Source Inverters Operating in GridConnected and Islanded Modes," IEEE transactions on industrial electronics, vol. 56, no. 10, pp. 4088-4096, October 2009.

[6] J. Jung, N. Vu, D. Dang, T. Do, Y. Choi and H. Choi, "A Three-Phase Inverter for a Standalone Distributed Generation System: Adaptive Voltage Control Design 
and Stability Analysis," IEEE transactions on energy conversion, vol. 29, no. 1, pp. 46-56, March 2014.

[7] J. C. Vasquez, J. M. Guerrero, M. Savaghebi, J. Eloy-Garcia and R. Teodorescu, "Modeling, Analysis, and Design of Stationary-Reference-Frame Droop-Controlled Parallel Three-Phase Voltage Source Inverters," IEEE transactions on industrial electronics, vol. 60, no. 4, pp. 1271-1280, April 2013.

[8] N. S. Nise, Control Systems Engineering, $6^{\text {th }}$ Edition, John Wiley and Sons Inc., Hoboken, NJ, 2011.

[9] L. Wu, Z. Zhao and J. Liu, "A Single-Stage Three-Phase Grid-Connected Photovoltaic System With Modified MPPT Method and Reactive Power Compensation," IEEE transactions on energy conversion, vol. 22, no. 4, pp. 881-886, December 2007

[10] E. Lavretsky and K. A. Wise, Robust and Adaptive Control with Aerospace Application, Springer, London, United Kingdom, 2013

[11] G. Tao, Adaptive Control Design and Analysis, John Wiley and Sons Inc., New York, NY, 2003.

[12] J. J. E. Slotine and W. Li, Applied Nonlinear Control, Prentice Hall Inc., Upper Saddle River, NJ, 1991.

[13] C. Chen, Linear System Theory and Design, Oxford University Press Inc., 1995.

[14] D. S. Bernstein, "A student's guide to classical control," IEEE Control Systems Magazine, vol. 17, no. 4, pp. 96-100, 1997.

[15] N. S. Nise, Control Systems Engineering, John Wiley and Sons Inc., $6^{\text {th }}$ Edition., Hoboken, NJ, 2011. 
[16] XC164 Different PWM Waveforms Generation for 3-Phase AC Induction Motor with XC164CS, Infineon Technologies AG 81726 Mnchen, Germany, 2006.

[17] R. S. Pedro, R. C. Carlos, R. C. Enrique, O. Husev and E. Makovenko, "Control Scheme of a Three-phase Three level NPC qz-Source Inverter with LCL filter for RES applications," Journal of Electrical Engineering \& Technonlogy, vol. 8, no. 3, pp. 544-558, 2013.

[18] F. Gonzalez-Espin, G. Gargera, I. Patrao and E. Figueres, "An Adaptive Control System for Three-phase Photovoltaic Incerters Working in a polluted and Variable Frequency Electric Grid," IEEE Transactions on Power Electronics, vol. 27, no. 10, pp. 4248-4261, October 2012.

[19] G. Rigatos, P. Siano, N. Zervos and C. Cecati, "Control of Three-Phase Voltage Source Converters with the Derivative-Free Nonlinear Kalman Filter," Intelligent Industrial Systems, vol. 2, no. 1, pp. 21-33, 2016.

[20] "Grid Connected PV Systems", Acmepoint Energy Services, Retrieved 28 April 2015.

[21] N. A. Rahim, R. Saidur, K. H. Solangi, M. Othman and N. Amin, "Survey of grid-connected photovoltaic inverters and related systems," Clean Techn Enciron Policy, vol. 14, pp. 521-533, 2012.

[22] S. Chung, "A phase tracking system for three phase utility interface inverters," IEEE Transactions on Industrial Electrionics, vol. 15, no. 3, pp. 431-438, 2000.

[23] A. Timbus, R. Teodoresecu and F. Blaabjerg, "Synchronization methods for three phase distributed power generation systems," Power Electronics Specialists Conference, pp.2474-2481, 2005. 
[24] L. Fraas and L. Partain, Solar Cells and Their Applications Second Edition, Section 10.2, Wiley, 2010.

[25] R. Teodorescu, F. Blaabjerg, M.Liserre and P.C. Loh, "Proportionalresonant controllers and filters for grid-connected voltage-source converters," IEE Proceedings-Electric Power Applications, vol. 1453, no. 5, pp. 750-762, September 2006.

[26] H. R. Karshenas and H. Saghafi, "Basic criteria in designing lcl filters for grid connected converters," IEEE International Symposium on Industrial Electronics, pp. 1996-2000, 2006

[27] R. Teodorescu, F. Blaabjerg and U. Borup, "A new control structure for grid connected LCL PC inverters with zero steady state error and selective harmonic compensation," Applied Power Electronics Conference and Exposition, 2004. APEC'04. Nineteenth Annual IEEE, pp. 22-26, February 2004.

[28] C. Kamalakannan and L. P. Suresh, "Design and Analysis of Three Phase Four Wire," Power Electronics and Renewable Energy Systems, NY: Springer India, pp.1029, 2015

[29] R. H. Park, "Two Reaction Theory of Synchronous Machines," AIEE Transactions vol.48, pp. 716-730, 1929.

[30] L. Chen, A. Amirahmadi, Q. Zhang, N. Kutkut, I. Batarseh, "Design and Implementation of Three-Phase Two-Stage Grid-Connected Module Integrated Converter," IEEE Transactions on Power Electronics, vol.28, no.9, August 2014.

[31] L. Wen, G. Tao, Y. Liu, "Aircraft Turbulence Compensation Using Adaptive Multivariable Disturbance Rejection Techniques," Journal of Guidance, Control, and Dynamics, vol. 28, no. 5, pp. 954-962 
[32] L. Wen, G. Tao, Y. Liu, "Multivariable adaptive output rejection of unmatched input disturbances," International Journal of Adaptive Control and Signal Processing, vol. 30, pp.1203-1227, 2016 